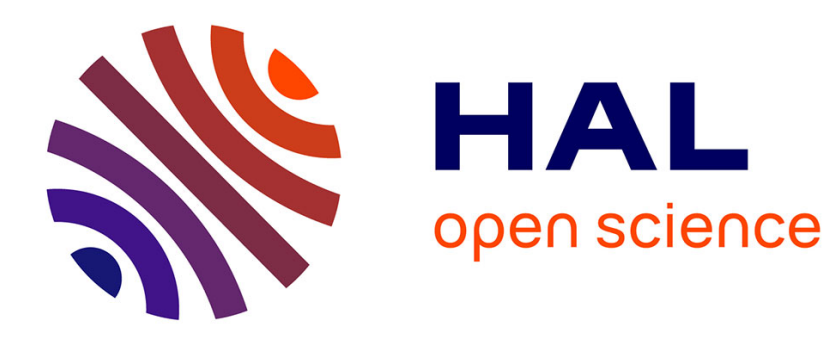

\title{
A GENERAL NOTION OF COHERENT SYSTEMS
}

\author{
Alexander Schmitt
}

\section{To cite this version:}

Alexander Schmitt. A GENERAL NOTION OF COHERENT SYSTEMS. 2021. hal-02391836v2

\section{HAL Id: hal-02391836 \\ https://hal.science/hal-02391836v2}

Preprint submitted on 26 Sep 2021

HAL is a multi-disciplinary open access archive for the deposit and dissemination of scientific research documents, whether they are published or not. The documents may come from teaching and research institutions in France or abroad, or from public or private research centers.
L'archive ouverte pluridisciplinaire HAL, est destinée au dépôt et à la diffusion de documents scientifiques de niveau recherche, publiés ou non, émanant des établissements d'enseignement et de recherche français ou étrangers, des laboratoires publics ou privés. 


\title{
A GENERAL NOTION OF COHERENT SYSTEMS
}

\author{
ALEXANDER SCHMITT
}

\begin{abstract}
AвSTRACT. We look at coherent systems for decorated vector bundles and propose a notion of semistability. In the special case of tensor powers, we will examine this notion more closely. In particular, we will construct moduli spaces with the help of geometric invariant theory. It is an interesting aspect that ampleness of the linearization in the geometric invariant theory construction yields a bound on the stability parameter for coherent systems.
\end{abstract}

\section{INTRODUCTION}

Classical Brill-Noether theory studies the existence of unusually large linear systems, called special, on smooth projective curves. For this, one has to analyze how the number $h^{0}(X, L)$ behaves as $X$ varies over the moduli space $\mathscr{M}_{g}$ of smooth projective curves of genus $g$ and $L$ varies over the Jacobian $\operatorname{Pic}^{d}(X)$ of line bundles of degree $d$ on $X, g$ and $d$ fixed. Brill-Noether theory gives precise information on the locus of pairs $(X, L)$ for which $h^{0}(X, L)$ exceeds a given number $s$ and the locus of curves $X$ of genus $g$ that admit a line bundle $L$ with $h^{0}(X, L) \geq s$. In this way, it is, e.g., possible to understand linear systems on general curves. An exposition of Brill-Noether theory in the context of modern algebraic geometry is given in the famous text [2].

It is natural to consider analogous questions for vector bundles of higher rank, possibly restricting to semistable or stable ones. To this end, Peter Newstead, the former chairman of the international research group "VBAC — Vector Bundles on Algebraic Curves", proposed the Brill-Noether project (https://www.liverpool.ac.uk/ newstead/bnt.html, see [32] for a list of open questions in this context). Brill-Noether theory for vector bundles of higher rank turned out to be substantially different from Brill-Noether theory for line bundles. To describe the relevant loci, it is natural to consider coherent systems, i.e., pairs $(E, \Gamma)$ which consist of a vector bundle $E$ on a smooth projective curve $X$ and a subspace $\Gamma \subset H^{0}(X, E)$. The project of classifying coherent systems is an interesting moduli problem in algebraic geometry. There is a notion of semistability for coherent systems which depends on a parameter, and, for each stability parameter, there is a projective moduli space. This theory has been developed independently by King and Newstead [24] and by Le Potier [27]. One may study these moduli spaces in their own right as in [7] and [8] or in connection with moduli spaces of semistable vector bundles as in [6] or [5].

Analogs to coherent systems may be studied for any kind of decorated vector or principal bundles. Brambila-Paz discussed the case of coherent Higgs bundles in [9]. Another promising special case arises for symmetric powers. In that case, we can treat, e.g., linear systems of conic or quadric bundles over curves. Moduli problems for these objects were studied, e.g., in [17]. We will make some remarks on that problem in Section 1.3. It is also discussed in [36], Section 5.1.

2020 Mathematics Subject Classification. 14H60, 14D20, 14L24.

Key words and phrases. Coherent system, moduli space, geometric invariant theory, semistability, linearization, ampleness. 
In this note, we will define semistability for coherent systems of decorated vector bundles. It depends on a positive rational number $\delta$. In the special case of decorated vector bundles associated with tensor powers of the standard representation of $\mathrm{GL}_{r}(\mathbb{C})$, we establish the following result.

Main Theorem. For a smooth projective curve $X$ over the complex numbers, $a, r>0$, $d \in \mathbb{Z}, W:=\mathbb{C}^{r}, \varrho: \mathrm{GL}_{r}(\mathbb{C}) \longrightarrow \mathrm{GL}\left(W^{\otimes a}\right)$ the a-th tensor power of the standard representation, and

$$
\delta<\frac{1}{a \cdot(a-1) \cdot r^{a-1}},
$$

there is a projective moduli scheme $\mathscr{C}_{X / \varrho / \mathscr{O}_{X} / d / s}^{\delta}$ for $\delta$-semistable coherent systems $(E, \Gamma)$, consisting of a vector bundle $E$ of rank $r$ and degree $d$ on $X$ and a linear subspace $\Gamma \subset H^{0}\left(X, E^{\otimes a}\right)$ of dimension $s$.

The bound $(\star)$ on the stability parameter is a novel feature. We need to impose it in order to perform the construction of moduli spaces with the help of geometric invariant theory. In the setting of decorated vector bundles, there does not exist such a bound. The notion of semistability simply stabilizes beyond some value $\delta_{\infty}$ (see [35], Proposition 2.3.6.5). ${ }^{1}$ In the case of parabolic vector bundles, there also exist some a priori bounds on the parameters ([37], Troisième Partie, Définition 2). The reason in that context is that beyond those bounds one needs to allow some coherent sheaves with torsion as well, but the moduli spaces still exist (compare [40]). It will be interesting to investigate what the precise meaning of the bound that we need to impose is. For example, if $r$ is fixed, the tensor power $a$ is very large, and the dimension $s$ of $\Gamma$ is rather small, the bound is so strong that the vector bundle underlying a semistable coherent system has to be itself semistable (see Example 1.5.1). On the other hand, we will explain in Section 4 how the approach $\delta \rightarrow 1 /\left(a \cdot(a-1) \cdot r^{a-1}\right)$ corresponds to the process of letting the stability parameter in [36] tend to infinity. To conclude this introduction, let us point out that new versions of geometric invariant theory have been developed which are based on the theory of stacks ([21], [1]) or affine Graßmannians [18]. In situations where a traditional approach via geometric invariant theory can be carried out, these techniques will lead to the same basic conclusions. Still, it will be interesting to try them out in the setting of general coherent systems.

Notation. We will work on a connected smooth projective curve $X$ of genus $g$ at least two which is defined over the field $\mathbb{C}$ of complex numbers, and we will fix a point $x_{0} \in X$. We write $\mathcal{O}_{X}(k)$ for $\mathcal{O}_{X}\left(k \cdot x_{0}\right)$, and, given a coherent $\mathcal{O}_{X}$-module $\mathscr{F}$, the symbol $\mathscr{F}(k)$ stands for the $\mathcal{O}_{X}$-module $\mathscr{F} \otimes_{\mathcal{O}_{X}} \mathcal{O}_{X}(k), k \in \mathbb{Z}$.

Given a scheme $S$ and a vector bundle $A$ on $S$, we write $P(A)$ for the projective bundle of lines in the fibers of $A$, i.e., for $\operatorname{Proj}\left(\operatorname{Sym}^{\star}\left(A^{\vee}\right)\right)$, and $\mathbb{P}(A)$ for $\operatorname{Proj}\left(\operatorname{Sym}^{\star}(A)\right)$.

For a cartesian product $A \times B$ in a category, we let $\pi_{A}: A \times B \longrightarrow \overline{A \text { and }} \overline{\pi_{B}}: A \times B \longrightarrow B$ be the natural projections.

Acknowledgments. I would like to thank Leticia Brambila-Paz for explaining to me the moduli problem of coherent Higgs bundles during the XII Coloquio Latinoamericano de Álgebra in Quito, 2017. Jochen Heinloth and Peter Newstead gave me important hints to the literature. My special thanks go to Georg Hein and Norbert Hoffmann for explaining to me the material around Proposition 2.4.6.

${ }^{1}$ This includes the case that there are no $\delta$-semistable objects, for $\delta>\delta_{\infty}$. 
Bradlow, Brambila-Paz, García-Prada, and Gothen and Brambila-Paz and Edgar I. Castañeda are carrying out independent research on the case of coherent Higgs systems. Their results will appear in forthcoming papers.

Last but not least, I am grateful to the referees for their various corrections and suggestions to improve the exposition.

\section{A general Version of COHERENT SyStems}

As recalled in the introduction, classical coherent systems were studied, e.g., in [24] and [27]. Bradlow, Brambila-Paz, García-Prada, and Gothen suggested a version of coherent systems for Higgs bundles. There is a nice candidate for a notion of semistability for these objects which generalizes the one for classical coherent systems given in [24]. We will state it in this section. However, the natural approach via geometric invariant theory requires some bounds on the stability parameter which we will make explicit for some representations.

1.1. Coherent systems. Let $\mathrm{GL}_{r}(\mathbb{C})$ be the linear algebraic group consisting of invertible $(r \times r)$-matrices, $a \in \mathbb{Z}, H$ a finite dimensional complex vector space, and $\varrho: \mathrm{GL}_{r}(\mathbb{C}) \longrightarrow$ $\mathrm{GL}(H)$ a homogeneous representation of degree $a$ of $\mathrm{GL}_{r}(\mathbb{C})$ on $H$. This means that, for $t \in \mathbb{C}^{\star}, \varrho\left(t \cdot \mathbb{E}_{r}\right)=t^{a} \cdot \mathrm{id}_{H}$.

Given a vector bundle $E$ of rank $r$ on $X$, we use $\varrho$ to associate with it the vector bundle $E_{\varrho}$ with typical fiber $H$. Finally, we fix a line bundle $L$ on $X$. Now, a coherent $\varrho$-system is a pair $(E, \Gamma)$ which consists of a vector bundle $E$ on $X$ of rank $r$ and a subspace $\Gamma \subset H^{0}\left(X, E_{\varrho} \otimes L\right)$. The triple $\left(L, \operatorname{deg}(E), \operatorname{dim}_{\mathbb{C}}(\Gamma)\right)$ is the type of $(E, \Gamma){ }^{2}$

Remark 1.1.1. i) Let $(E, \Gamma)$ be a coherent $\varrho$-system. The evaluation map gives a homomorphism $\varphi: \Gamma \otimes \mathcal{O}_{X} \longrightarrow E_{\varrho}$. The pair $(E, \varphi)$ is a swamp for the representation $\varrho^{\oplus \operatorname{dim}_{C}(\Gamma)}$ (see [35], p. 136).

ii) For $\varrho=\operatorname{id}_{\mathrm{GL}_{r}(\mathbb{C})}$ and $L=\mathscr{O}_{X}$, we obtain the coherent systems studied, e.g., in [24] and [8].

1.2. Some numerical quantities. As for bumps (see [35], p. 116), the test objects for semistability are weighted filtrations. Recall that, for a vector bundle $F$ on $X$, a weighted filtration is a pair $\left(F_{\bullet}, \alpha_{\bullet}\right)$ in which

$$
F_{\bullet}: \quad\{0\} \subsetneq F_{1} \subsetneq \cdots \subsetneq F_{l} \subsetneq F
$$

is a filtration of $F$ by subbundles and

$$
\alpha_{\bullet}=\left(\alpha_{1}, \ldots, \alpha_{l}\right)
$$

is a tuple of positive rational numbers.

Now, let $(E, \Gamma)$ be a coherent $\varrho$-system and $\left(E_{\bullet}, \alpha_{\bullet}\right)$ a weighted filtration of $E$. It induces a weighted filtration $\left(B_{\bullet}, \beta_{\bullet}\right)$ of $E_{\varrho}$ (compare [35], p. 176, and the proof of Proposition 3.4.3, below). Here, we write

$$
B_{\bullet}: \quad\{0\} \subsetneq B_{1} \subsetneq \cdots \subsetneq B_{m} \subsetneq E_{\varrho},
$$

set

and

$$
b_{j}:=\operatorname{dim}_{\mathbb{C}}\left(H^{0}\left(X, B_{j} \otimes L\right) \cap \Gamma\right), \quad j=1, \ldots, m,
$$

$$
\mu_{(E, \Gamma)}\left(E_{\bullet}, \alpha_{\bullet}\right):=\sum_{j=1}^{m} \beta_{j} \cdot\left(\operatorname{rk}\left(B_{j}\right) \cdot \operatorname{dim}_{\mathbb{C}}(\Gamma)-b_{j} \cdot \operatorname{rk}\left(E_{\varrho}\right)\right) .
$$

${ }^{2}$ The rank of $E$ is encoded in the representation $\varrho$. 
Remark 1.2.1. Let $0<s<\operatorname{dim}_{\mathbb{C}}(H)$. Via $\varrho, \mathrm{GL}_{r}(\mathbb{C})$ acts on $H$ and, so, also on the Graßmannian $\mathfrak{G r}_{s}(H)$ of $s$-dimensional subspaces of $H$. Pick $\Delta \in \mathfrak{G r}_{S}(H)$ and let $\lambda: \mathbb{C}^{\star} \longrightarrow \mathrm{SL}_{r}(\mathbb{C})$ be a one parameter subgroup. The one parameter subgroup $\lambda$ defines a weighted flag $\left(W_{\bullet}, \alpha_{\bullet}\right)$ inside $\mathbb{C}^{r}$ ([35], Example 1.5.1.36). It was observed by Mumford ([30], Chapter 2, Proposition 2.7, [35], Proposition 1.5.1.35) that $\mu(\lambda, \Delta)$ depends only on $\left(W_{\bullet}, \alpha_{\bullet}\right)$. Now, the one parameter subgroup $\varrho \circ \lambda$ of $\operatorname{SL}(H)$ defines a weighted flag $\left(H_{\bullet}, \beta_{\bullet}\right)$ inside $H$. Standard computations (e.g., [30], Chapter 4, §4, [31], Chapter 4, §6, [35], Exercise 1.5.1.15) show that

$$
\mu(\lambda, \Delta)=\sum_{j=1}^{m} \beta_{j} \cdot\left(\operatorname{dim}_{\mathbb{C}}\left(H_{j}\right) \cdot \operatorname{dim}_{\mathbb{C}}(\Delta)-\operatorname{dim}_{\mathbb{C}}\left(H_{j} \cap \Delta\right) \cdot \operatorname{dim}_{\mathbb{C}}(H)\right) .
$$

This explains to some extent the origin of $\mu_{(E, \Gamma)}\left(E_{\bullet}, \alpha_{\bullet}\right)$.

As in [35], p. 139, we define

$$
M\left(E_{\bullet}, \alpha_{\bullet}\right):=\sum_{i=1}^{l} \alpha_{i} \cdot\left(\operatorname{deg}(E) \cdot \operatorname{rk}\left(E_{i}\right)-\operatorname{deg}\left(E_{i}\right) \cdot r\right) .
$$

1.3. Coherent systems of conic bundles. Let us look at the representation $\varrho$ of $\mathrm{GL}_{r}(\mathbb{C})$ on the space $\operatorname{Sym}^{2}\left(\left(\mathbb{C}^{r}\right)^{\vee}\right)$. It corresponds to the action

$$
\begin{aligned}
\sigma: \operatorname{GL}_{r}(\mathbb{C}) \times \operatorname{Sym}_{r}(\mathbb{C}) & \longrightarrow \operatorname{Sym}_{r}(\mathbb{C}) \\
(g, m) & \longmapsto\left(g^{-1}\right)^{t} \cdot m \cdot g^{-1}
\end{aligned}
$$

of $\mathrm{GL}_{r}(C)$ on the vector space $\operatorname{Sym}_{r}(\mathbb{C})$ of symmetric $(r \times r)$-matrices. So, a coherent $\varrho$ system consists of a vector bundle $E$ on $X$ of rank $r$ and a subspace $\Gamma \subset \operatorname{Hom}\left(\operatorname{Sym}^{2}(E), L\right)$.

To get a concrete example, let us have a look at the case $r=2$. There is the one parameter subgroup

$$
\begin{aligned}
\lambda: \mathbb{C}^{\star} & \longrightarrow \mathrm{SL}_{2}(\mathbb{C}) \\
z & \longmapsto\left(\begin{array}{cc}
z^{-1} & 0 \\
0 & z
\end{array}\right) .
\end{aligned}
$$

Note

$$
\forall m=\left(\begin{array}{ll}
a & c \\
c & b
\end{array}\right) \in \operatorname{Sym}_{2}(\mathbb{C}), z \in \mathbb{C}^{\star}: \quad \lambda(z)^{-1} \cdot m \cdot \lambda(z)^{-1}=\left(\begin{array}{cc}
z^{2} \cdot a & c \\
c & z^{-2} \cdot d
\end{array}\right) .
$$

We find the filtration

$$
\{0\} \subsetneq\left\{\left(\begin{array}{cc}
0 & 0 \\
0 & \star
\end{array}\right)\right\} \subsetneq\left\{\left(\begin{array}{cc}
0 & \star \\
\star & \star
\end{array}\right)\right\} \subsetneq \operatorname{Sym}_{2}(\mathbb{C})
$$

and the weights

$$
\beta_{1}=\beta_{2}=\frac{2}{3}
$$

Now, let $E$ be a vector bundle of rank two on $X$ and $N \subsetneq E$ a line subbundle. It yields the weighted filtration $\left(E_{\bullet}, \alpha_{\bullet}\right)$ with

$$
E_{\bullet}: \quad\{0\} \subsetneq N \subsetneq E \text { and } \alpha_{\bullet}=(1) .
$$

The space $H^{0}\left(X, B_{1} \otimes L\right)$ is the space of all symmetric forms $\varphi: \operatorname{Sym}^{2}(E) \longrightarrow L$ in $\Gamma$ for which $N$ is contained in the radical, i.e., $\varphi(N \cdot E)=0$, and $H^{0}\left(X, B_{2} \otimes L\right)$ consists of all symmetric forms $\varphi: \operatorname{Sym}^{2}(E) \longrightarrow L$ in $\Gamma$ for which $N$ is isotropic, i.e., $\varphi(N \cdot N)=0$. A similar discussion applies to arbitrary rank and one step filtrations. The reader may compare this to [36], Lemma 5.2. 
1.4. Semistability. Let $\delta$ be a positive rational number. Using the quantities introduced in Section 1.2 , we say that $(E, \Gamma)$ is $\delta$-(semi)stable, if the inequality

$$
M\left(E_{\bullet}, \alpha_{\bullet}\right)+\delta \cdot \mu_{(E, \Gamma)}\left(E_{\bullet}, \alpha_{\bullet}\right)(\geq) 0
$$

is satisfied, for every weighted filtration $\left(E_{\bullet}, \alpha_{\bullet}\right)$ of $E$.

Example 1.4.1. Suppose that $\varrho=\mathrm{id}: \mathrm{GL}_{r}(\mathbb{C}) \longrightarrow \mathrm{GL}\left(\mathbb{C}^{r}\right)$ is the standard representation and $L=\mathscr{O}_{X}$. Then, a coherent $\varrho$-system is a coherent system in the usual sense, i.e., a pair $(E, \Gamma)$ consisting of a vector bundle $E$ on $X$ and a subspace $\Gamma \subset H^{0}(X, E)$. For a weighted filtration $\left(E_{\bullet}, \alpha_{\bullet}\right)$ of $E$, we get

$$
\mu_{(E, \Gamma)}\left(E_{\bullet}, \alpha_{\bullet}\right)=\sum_{i=1}^{l} \alpha_{i} \cdot\left(\operatorname{rk}\left(E_{i}\right) \cdot \operatorname{dim}_{\mathbb{C}}(\Gamma)-\operatorname{dim}_{\mathbb{C}}\left(H^{0}\left(X, E_{i}\right) \cap \Gamma\right) \cdot \operatorname{rk}(E)\right) .
$$

Apparently, we only need to check weighted filtrations of the shape $(\{0\} \subsetneq F \subsetneq E$, (1)). So, for $\delta \in \mathbb{Q}_{>0}$, the coherent system $(E, \Gamma)$ is $\delta$-(semi)stable if and only if the inequality ${ }^{3}$

$$
\frac{\operatorname{deg}(F)+\delta \cdot \operatorname{dim}_{\mathbb{C}}\left(H^{0}(X, F) \cap \Gamma\right)}{\operatorname{rk}(F)}(\leq) \frac{\operatorname{deg}(E)+\delta \cdot \operatorname{dim}_{\mathbb{C}}\left(H^{0}(X, E)\right)}{\operatorname{rk}(E)}
$$

is satisfied, for every non-trivial, proper subbundle $F$ of $E$. This is equivalent to the notion stated in [24], Definition 2.3.2, and [27], Définition 4.2.

1.5. Semistability for small parameters. If the representation $\varrho$ and the type are fixed, the stability parameter $\delta$ is small, and $(E, \Gamma)$ is a $\delta$-semistable coherent $\varrho$-system, then the vector bundle $E$ itself has to be semistable. More precisely, if $\delta \ll 1$, then a coherent $\varrho$-system $(E, \Gamma)$ of type $(L, d, s)$ is $\delta$-(semi)stable if and only if

(a) the vector bundle $E$ is semistable,

(b) and, for every weighted filtration $\left(E_{\bullet}, \alpha_{\bullet}\right)$, such that $M\left(E_{\bullet}, \alpha_{\bullet}\right)=0$, i.e., such that $\mu\left(E_{i}\right)=\mu(E)=d / r, i=1, \ldots, l$, the inequality

$$
\mu_{(E, \Gamma)}\left(E_{\bullet}, \alpha_{\bullet}\right)(\geq) 0
$$

holds true.

Example 1.5.1. Suppose $L=\mathcal{O}_{X}, a>0, W=\mathbb{C}^{r}$, and that $\varrho: \mathrm{GL}_{r}(\mathbb{C}) \longrightarrow \mathrm{GL}\left(W^{\otimes a}\right)$ is the $a$-th tensor power of the standard representation. In Section 3.1, we will see that, for a $\delta$-semistable coherent $\varrho$-system $(E, \Gamma)$ of type $\left(\mathcal{O}_{X}, d, s\right)$ and a subbundle $\{0\} \subsetneq F \subsetneq E$, one has

$$
\mu(F) \leq \mu(E)+\delta \cdot a \cdot s .
$$

Now, $\mu(F)-\mu(E) \in \mathbb{Z}[1 /(\operatorname{rk}(F) \cdot \operatorname{rk}(E))]$. So, if

$$
\delta<\frac{1}{a \cdot(r-1) \cdot r \cdot s},
$$

then $\delta$-semistability is equivalent to the conditions (a) and (b) stated above.

${ }^{3}$ The symbol " $(\leq)$ " in conjuction with "(semi)stable" means that the notions "semistable" and "stable" are being defined and that " $\leq$ " is used in the definition of "semistable" and " $<$ " in the definition of "stable". 
1.6. Statement of the main result. Fix the representation $\varrho$, the type $(L, d, s)$, and the stability parameter $\delta$. For a scheme $S$ of finite type over $\mathbb{C}$, a family of coherent $\varrho$-systems parameterized by $S$ is a family $\left(E_{S}, \varphi_{S}\right)$, consisting of a vector bundle $E_{S}$ of rank $r$ on $S \times X$ and a homomorphism $\varphi_{S}: \mathcal{O}_{S \times X}^{\oplus S} \longrightarrow E_{S, \varrho} \otimes \pi_{X}^{\star}(L)$, such that

$$
\pi_{S \star}\left(\varphi_{S}\right): \mathcal{O}_{S}^{\oplus s} \longrightarrow \pi_{S \star}\left(E_{S, \varrho} \otimes \pi_{X}^{\star}(L)\right)
$$

is universally injective (see [36], p. 27). Two families $\left(E_{S}^{1}, \varphi_{S}^{1}\right)$ and $\left(E_{S}^{2}, \varphi_{S}^{2}\right)$ parameterized by $S$ are isomorphic, if there are isomorphisms $\psi_{S}: E_{S}^{1} \longrightarrow E_{S}^{2}$ and $\lambda_{S} \in \Gamma\left(S, \mathrm{GL}_{S}\left(\mathcal{O}_{S}\right)\right)$, such that

$$
\varphi_{S}^{2}=\left(\psi_{S, \varrho} \otimes \mathrm{id}_{\pi_{X}^{\star}(L)}\right) \circ \varphi_{S}^{1} \circ \pi_{S}^{\star}\left(\lambda_{S}^{-1}\right) .
$$

The moduli functor $C_{X / \varrho / L / d / s}^{\delta}: \underline{\mathrm{Sch}}_{\mathbb{C}} \longrightarrow \underline{\text { Sets }}$ assigns to a scheme $S$ of finite type over $\mathbb{C}$ the set of isomorphy classes of families of $\delta$-semistable coherent $\varrho$-systems of type $(L, d, s)$ parameterized by $S$. It is somewhat similar to the moduli functor for decorated vector bundles studied in [34] and [35] (compare Remark 1.1.1). As in the case of decorated vector bundles, the existence of a coarse moduli space for this functor may be deduced from the existence of a certain categorical quotient (see Section 3.2 and 3.3). For proving the existence of the respective quotient, one may use geometric invariant theory. We will carry this out for tensor powers of the standard representation. The techniques should extend to all homogeneous representations.

Theorem 1.6.1. For $a>0, W:=\mathbb{C}^{r}$, and $\varrho: \mathrm{GL}_{r}(\mathbb{C}) \longrightarrow \mathrm{GL}\left(W^{\otimes a}\right)$ the a-th tensor power of the standard representation, the coarse moduli space $\mathscr{C}_{X / \varrho / \mathscr{O}_{X} / d / s}^{\delta}$ for the functor $C_{X / \varrho / \mathscr{O}_{X} / d / s}^{\delta}$ exists as a projective scheme, provided that

$$
\delta<\frac{1}{a \cdot(a-1) \cdot r^{a-1}} .
$$

The bound on $\delta$ grants that the line bundle in which we linearize the group action is ample, so that the Hilbert-Mumford criterion may be applied for determining the semistable points.

\section{Preliminaries from geOmetric inVariant theory}

We will first recall some basic facts from geometric invariant theory. Afterwards, we will discuss linearized line bundles on quot schemes in detail. This will be important to understand the bound given in Section 1.6 (see Section 3.3.5).

2.1. Weighted flags. Let $\lambda: \mathbb{C}^{\star} \longrightarrow \mathrm{SL}_{r}(\mathbb{C})$ be a one parameter subgroup. It is diagonalizable, i.e., there exist a basis $\underline{v}=\left(v_{1}, \ldots, v_{r}\right)$ of $\mathbb{C}^{r}$ and integers $g_{1} \leq \cdots \leq g_{r}$, such that

$$
\forall t \in \mathbb{C}^{\star}: \quad \lambda(t)\left(\sum_{i=1}^{r} \kappa_{i} \cdot v_{i}\right)=\sum_{i=1}^{r} t^{g_{i}} \cdot \kappa_{i} \cdot v_{i} .
$$

Now, let $\gamma_{1}<\cdots<\gamma_{l+1}$ be the distinct weights occuring in $\left\{g_{1}, \ldots, g_{r}\right\}$,

$$
l_{i}:=\max \left\{j=1, \ldots, t \mid g_{j}=\gamma_{i}\right\}, \quad i=1, \ldots, l+1,
$$

and

The flag

$$
V_{i}:=\left\langle v_{1}, \ldots, v_{l_{i}}\right\rangle, \quad i=1, \ldots, l+1
$$

$$
V_{\bullet}: \quad\{0\}=: V_{0} \subsetneq V_{1} \subsetneq \cdots \subsetneq V_{l} \subsetneq V_{l+1}=\mathbb{C}^{r}
$$


does not depend on the choice of the basis $\underline{v}$ for which (1) holds. Setting $\gamma_{\bullet}:=\left(\gamma_{1}, \ldots, \gamma_{l+1}\right)$, we call $\left(V_{\bullet}, \gamma_{\bullet}\right)$ the $\mathbb{Z}$-weighted flag associated with $\lambda$.

The $i$-th basic weight vector is

$$
g_{r}^{(i)}:=(\underbrace{i-r, \ldots, i-r}_{i \times}, \underbrace{i, \ldots, i}_{(r-i) \times}), \quad i=1, \ldots, r-1 .
$$

Since $\lambda$ is a one parameter subgroup of the special linear group, the important identity

$$
\left(g_{1}, \ldots, g_{r}\right)=\sum_{i=1}^{r-1} \frac{g_{i+1}-g_{i}}{r} \cdot g_{r}^{(i)}=\sum_{i=1}^{l} \frac{\gamma_{i+1}-\gamma_{i}}{r} \cdot g_{r}^{\left(l_{i}\right)}
$$

holds true. We set $\alpha_{i}:=\left(\gamma_{i+1}-\gamma_{i}\right) / r, i=1, \ldots, l, \alpha_{\bullet}:=\left(\alpha_{1}, \ldots, \alpha_{l}\right)$, and call $\left(V_{\bullet}, \alpha_{\bullet}\right)$ the $\mathbb{Q}_{>0}$-weighted flag associated with $\lambda$.

2.2. Parabolic subgroups. Let $H$ be a semisimple linear algebraic group. For a nonconstant one parameter subgroup $\lambda: \mathbb{C}^{\star} \longrightarrow H$, we define

$$
Q_{H}(\lambda):=\left\{g \in G \mid \lim _{z \rightarrow \infty} \lambda(z) \cdot g \cdot \lambda(z)^{-1} \text { exists in } H\right\} .
$$

This is a parabolic subgroup of $H .{ }^{4}$ Fix a maximal torus $T \subset H$ and let $X_{\star}(T)$ be the abelian group of homomorphisms from $\mathbb{C}^{\star}$ to $T$. We also introduce $X_{\star, \mathbb{Q}}(T):=X_{\star}(T) \underset{\mathbb{Z}}{\otimes} \mathbb{Q}$ and $X_{\star, \mathbb{R}}(T):=X_{\star}(T) \underset{\mathbb{Z}}{\otimes}$. Since, for any positive integer $k$ and any one parameter subgroup $\lambda \in X_{\star}(T)$, we have $Q_{H}(k \cdot \lambda)=Q_{H}(\lambda)$, we may define $Q_{H}(\ell)$, for any element $\ell \in X_{\star, \mathbb{Q}}(T)$. We fix a Borel subgroup $T \subset B \subset H$, set

$$
\overline{\mathscr{W}}_{\mathbb{Q}}(B, T):=\left\{\ell \in X_{\star, \mathbb{Q}}(T) \mid B \subset Q_{H}(\ell)\right\},
$$

and let $\overline{\mathscr{W}}_{\mathbb{R}}(B, T)$ be the closure of $\overline{\mathscr{W}}_{\mathbb{Q}}(B, T)$ in $X_{\star, \mathbb{R}}(T)$. We call this, slightly abusively, a Weyl chamber. This is a rational polyhedral cone, and the edges of $\overline{\mathscr{W}}_{\mathbb{Q}}(B, T)$ correspond to the maximal parabolic subgroups of $H$ that contain $B$. If we let $B$ run through the (finitely many) Borel subgroups containing $T$, we get a decomposition

$$
X_{\star, \mathbb{R}}(T)=\bigcup_{T \subset B \subset H} \overline{\mathscr{W}}_{\mathbb{R}}(B, T) .
$$

Example 2.2.1. i) Let $H$ be $\mathrm{SL}_{N}(\mathbb{C}), T$ the torus consisting of the diagonal matrices, and $\left(e_{1}, \ldots, e_{N}\right)$ the standard basis. For each permutation $\sigma \in \mathbb{S}_{N}$, we get the full flag

$$
W_{\bullet}(\sigma): \quad\{0\} \subsetneq\left\langle e_{\sigma(1)}\right\rangle \subseteq \cdots \subset\left\langle e_{\sigma(1)}, \ldots, e_{\sigma(N-1)}\right\rangle \subsetneq \mathbb{C}^{N} .
$$

The stabilizer $B_{\sigma}$ of $W_{\bullet}(\sigma)$ is a Borel subgroup, and $\sigma \longmapsto B_{\sigma}$ establishes a bijection between $\mathfrak{S}_{N}$ and the set of Borel subgroups containing $T$. Now, $\lambda \in X_{\star}(T)$ lies in $\overline{\mathscr{W}}_{\mathbb{R}}\left(B_{\sigma}, T\right)$ if and only if the flag $W_{\bullet}$ associated with $\lambda$ satisfies $W_{i}=\left\langle e_{\sigma(1)}, \ldots, e_{\sigma\left(\operatorname{dim}_{\mathbb{C}}\left(W_{i}\right)\right)}\right\rangle$, $i=1, \ldots, l$. The edges of $\overline{\mathscr{W}}_{\mathbb{R}}\left(B_{\sigma}, T\right)$ are spanned by the one parameter subgroups $\lambda_{\sigma}(j)$, $j=1, \ldots, N-1$, where $\lambda_{\sigma}(j)$ is the one parameter subgroup that satisfies (1) with respect to $v_{i}=e_{\sigma(i)}, i=1, \ldots, N$, and $\left(g_{1}, \ldots, g_{N}\right)=g_{N}^{(j)}, j=1, \ldots, N-1$.

ii) Let $\left(W_{\bullet}^{\prime}, \beta_{\bullet}^{\prime}\right)$ and $\left(W_{\bullet}, \beta_{\bullet}\right)$ be two $\mathbb{Q}_{>0}$-weighted flags of $\mathbb{C}^{N}$ with

$$
\begin{array}{ll}
W_{\bullet}^{\prime}: & \{0\}=: W_{0}^{\prime} \subsetneq W_{1}^{\prime} \subsetneq \cdots \subsetneq W_{l^{\prime}}^{\prime} \subsetneq W_{l^{\prime}+1}=\mathbb{C}^{N}, \\
W_{\bullet}: & \{0\}=: W_{0} \subsetneq W_{1} \subsetneq \cdots \subsetneq W_{l} \subsetneq W_{l+1}=\mathbb{C}^{N},
\end{array}
$$

${ }^{4}$ The same definition may be used for arbitrary reductive linear algebraic groups. If $\lambda$ maps to the center of the group, then the subgroup associated with $\lambda$ is the whole group. 
$\beta_{\bullet}^{\prime}=\left(\beta_{1}^{\prime}, \ldots, \beta_{l^{\prime}}^{\prime}\right)$, and $\beta_{\bullet}=\left(\beta_{1}, \ldots, \beta_{l}\right)$. We write $\left(W_{\bullet}^{\prime}, \beta_{\bullet}^{\prime}\right) \leq\left(W_{\bullet}, \beta_{\bullet}\right)$ if $l^{\prime} \leq l$ and there is a strictly increasing map $\iota:\left\{1, \ldots, l^{\prime}\right\} \longrightarrow\{1, \ldots, l\}$, such that

$$
\forall k \in\left\{1, \ldots, l^{\prime}\right\}: \quad W_{k}^{\prime}=W_{\iota(k)} \quad \text { and } \quad \beta_{k}^{\prime}=\beta_{\iota(k)} .
$$

Note that $\iota$ is unique in this case. Now, let $\lambda^{\prime}, \lambda \in \overline{\mathscr{W}}_{\mathbb{R}}(B, T) \cap X_{\star}(T)$. Then, there is a unique face $\mathscr{F}$ of $\overline{\mathscr{W}}_{\mathbb{R}}(B, T)$, such that $\lambda$ is contained in the relative interior of $\mathscr{F}$. Suppose that $\lambda^{\prime}$ lies in $\mathscr{F}$. Then, the $\mathbb{Q}_{>0}$-weighted flags $\left(W_{\bullet}^{\prime}, \beta_{\bullet}^{\prime}\right)$ and $\left(W_{\bullet}, \beta_{\bullet}\right)$ associated with $\lambda^{\prime}$ and $\lambda$, respectively, satisfy $\left(W_{\bullet}^{\prime}, \beta_{\bullet}^{\prime}\right) \leq\left(W_{\bullet}, \beta_{\bullet}\right)$.

iii) Let $\left(W_{\bullet}^{\prime}, \beta_{\bullet}^{\prime}\right),\left(W_{\bullet}^{\prime \prime}, \beta_{\bullet}^{\prime \prime}\right)$, and $\left(W_{\bullet}, \beta_{\bullet}\right)$ be three $\mathbb{Q}_{>}>0$-weighted flags of length $l^{\prime}, l^{\prime \prime}$, and $l$, respectively. We write

$$
\left(W_{\bullet}, \beta_{\bullet}\right)=\left(W_{\bullet}^{\prime}, \beta_{\bullet}^{\prime}\right)+\left(W_{\bullet}^{\prime \prime}, \beta_{\bullet}^{\prime \prime}\right)
$$

if

- $\left(W_{\bullet}^{\prime}, \beta_{\bullet}^{\prime}\right) \leq\left(W_{\bullet}, \beta_{\bullet}\right)$ and $\left(W_{\bullet}^{\prime \prime}, \beta_{\bullet}^{\prime \prime}\right) \leq\left(W_{\bullet}, \beta_{\bullet}\right)$,

- $\{1, \ldots, l\}=\operatorname{Im}\left(\iota^{\prime}\right) \cup \operatorname{Im}\left(\iota^{\prime \prime}\right), \iota^{\prime}:\left\{1, \ldots, l^{\prime}\right\} \longrightarrow\{1, \ldots, l\}$ and $\iota^{\prime \prime}:\left\{1, \ldots, l^{\prime \prime}\right\} \longrightarrow$ $\{1, \ldots, l\}$ being the respective strictly increasing maps,

- and, for $k \in\{1, \ldots, l\}$,

$$
\beta_{k}=\left\{\begin{aligned}
\beta_{k^{\prime}}^{\prime}, & \text { if } k=\iota^{\prime}\left(k^{\prime}\right) \text { and } k \notin \operatorname{Im}\left(\iota^{\prime \prime}\right) \\
\beta_{k^{\prime \prime}}^{\prime \prime}, & \text { if } k=\iota^{\prime \prime}\left(k^{\prime \prime}\right) \text { and } k \notin \operatorname{Im}\left(\iota^{\prime}\right) . \\
\beta_{k^{\prime}}^{\prime}+\beta_{k^{\prime \prime}}^{\prime \prime}, & \text { if } k=\iota^{\prime}\left(k^{\prime}\right)=\iota^{\prime \prime}\left(k^{\prime \prime}\right)
\end{aligned}\right.
$$

Suppose that $\lambda^{\prime}, \lambda^{\prime \prime}, \lambda \in \overline{\mathscr{W}}_{\mathbb{R}}(B, T) \cap X_{\star}(T)$ and $\lambda=\lambda^{\prime}+\lambda^{\prime \prime}$. Then, the $\mathbb{Q}_{>0}$-weighted flags $\left(W_{\bullet}^{\prime}, \beta_{\bullet}^{\prime}\right),\left(W_{\bullet}^{\prime \prime}, \beta_{\bullet}^{\prime \prime}\right)$, and $\left(W_{\bullet}, \beta_{\bullet}\right)$ associated with $\lambda^{\prime}, \lambda^{\prime \prime}$, and $\lambda$, respectively, satisfy

$$
\left(W_{\bullet}, \beta_{\bullet}\right)=\left(W_{\bullet}^{\prime}, \beta_{\bullet}^{\prime}\right)+\left(W_{\bullet}^{\prime \prime}, \beta_{\bullet}^{\prime \prime}\right)
$$

Now, suppose $G$ is another semisimple linear algebraic group, $\eta: G \longrightarrow H$ is a homomorphism, and $S \subset G$ is a maximal torus with $\eta(S) \subset T$. It is clear that we may find rational polyhedral cones $\overline{\mathscr{V}}_{1}, \ldots, \overline{\mathscr{V}}_{K} \subset X_{\star, \mathbb{R}}(S)$, such that

- for each $k \in\{1, \ldots, K\}$, there are a Borel subgroup $S \subset A \subset G$ and a Borel subgroup $T \subset B \subset H$, such that $\overline{\mathscr{V}}_{k} \subset \overline{\mathscr{W}}(A, S)$ and $\eta_{\star, \mathbb{R}}\left(\overline{\mathscr{V}}_{k}\right) \subset \overline{\mathscr{W}}(B, T)$, $\eta_{\star, \mathbb{R}}: X_{\star, \mathbb{R}}(S) \longrightarrow X_{\star, \mathbb{R}}(T)$ being the $\mathbb{R}$-linear map induced by $\eta$,

- $X_{\star, \mathbb{R}}(S)=\bigcup_{k=1}^{N} \overline{\mathscr{V}}_{k}$,

- for $k, k^{\prime} \in K$, the intersection $\overline{\mathscr{V}}_{k} \cap \overline{\mathscr{V}}_{k^{\prime}}$ is a common face of both $\overline{\mathscr{V}}_{k}$ and $\overline{\mathscr{V}}_{k}^{\prime}$.

For the rest of this section, we assume that $G$ is $\mathrm{SL}_{r}(\mathbb{C}), H$ is $\mathrm{SL}_{N}(\mathbb{C})$, and that $\eta: \mathrm{SL}_{r}(\mathbb{C}) \longrightarrow \mathrm{SL}_{N}(\mathbb{C})$ is induced by a homogeneous representation $\varrho: \mathrm{GL}_{r}(\mathbb{C}) \longrightarrow$ $\mathrm{GL}_{N}(\mathbb{C})$. Let $S \subset \mathrm{SL}_{r}(\mathbb{C})$ be the sugroup of diagonal matrices. After applying an inner automorphism of $\mathrm{GL}_{N}(\mathbb{C})$, if necessary, we may assume that $\eta(S)=\varrho(S)$ is contained in the torus $T \subset \mathrm{SL}_{N}(\mathbb{C})$ of diagonal matrices. Now, suppose that $\lambda^{\prime}, \lambda^{\prime \prime}, \lambda \in X_{\star, \mathbb{Q}}(S)$ are one parameter subgroups, such that $\lambda=\lambda^{\prime}+\lambda^{\prime \prime}$ and there exists an index $k \in\{1, \ldots, K\}$ with $\lambda^{\prime}, \lambda^{\prime \prime} \in \overline{\mathscr{V}}_{k}$. Clearly, also $\lambda \in \overline{\mathscr{V}}_{k}$. Now, $\lambda^{\prime}, \lambda^{\prime \prime}$, and $\lambda$ define $\mathbb{Q}_{>}$-weighted flags $\left(V_{\bullet}^{\prime}, \alpha_{\bullet}^{\prime}\right),\left(V_{\bullet}^{\prime \prime}, \alpha_{\bullet}^{\prime \prime}\right)$, and $\left(V_{\bullet}, \alpha_{\bullet}\right)$, respectively, and, as pointed out in Example 2.2.1, iii),

$$
\left(V_{\bullet}, \alpha_{\bullet}\right)=\left(V_{\bullet}^{\prime}, \alpha_{\bullet}^{\prime}\right)+\left(V_{\bullet}^{\prime \prime}, \alpha_{\bullet}^{\prime \prime}\right) \text {. }
$$

Likewise, $\eta_{\star}\left(\lambda^{\prime}\right), \eta_{\star}\left(\lambda^{\prime \prime}\right)$, and $\eta_{\star}(\lambda)$ define $\mathbb{Q}_{>0}$-weighted flags $\left(W_{\bullet}^{\prime}, \beta_{\bullet}^{\prime}\right),\left(W_{\bullet}^{\prime \prime}, \beta_{\bullet}^{\prime \prime}\right)$, and $\left(W_{\bullet}, \beta_{\bullet}\right)$. Since $\eta_{\star}\left(\lambda^{\prime}\right)+\eta_{\star}\left(\lambda^{\prime \prime}\right)=\eta_{\star}(\lambda)$, and, by construction, there is a Borel subgroup $T \subset B \subset \mathrm{SL}_{N}(\mathbb{C})$ with $\eta_{\star}\left(\lambda^{\prime}\right), \eta_{\star}\left(\lambda^{\prime \prime}\right), \eta_{\star}(\lambda) \in \overline{\mathscr{W}}(B, T)$, we infer

$$
\left(W_{\bullet}, \beta_{\bullet}\right)=\left(W_{\bullet}^{\prime}, \beta_{\bullet}^{\prime}\right)+\left(W_{\bullet}^{\prime \prime}, \beta_{\bullet}^{\prime \prime}\right)
$$


For $\lambda \in X_{\star, \mathbb{Q}}(S)$ with associated $\mathbb{Q}_{>0}$-weighted flag $\left(V_{\bullet}:\{0\} \subsetneq V_{1} \subsetneq \cdots \subsetneq V_{l} \subsetneq \mathbb{C}^{r}, \alpha_{\bullet}\right)$, define the type $t(\lambda):=\left(r_{\bullet}, \alpha_{\bullet}\right)$ by

$$
r_{\bullet}:=\left(r_{1}, \ldots, r_{l}\right), \quad r_{i}:=\operatorname{dim}_{\mathbb{C}}\left(V_{i}\right), \quad i=1, \ldots, l .
$$

Finally, let $\lambda_{1}, \ldots, \lambda_{L} \in X_{\star}(S)$ be the one parameter subgroups that occur as an indivisible generator of an edge of a cone $\overline{\mathscr{V}}_{k} \subset \overline{\mathscr{W}}(A, S), k \in\{1, \ldots, K\}$. We set ${ }^{5}$

$$
\mathscr{T}(\varrho):=\left\{t\left(\lambda_{1}\right), \ldots, t\left(\lambda_{L}\right)\right\} .
$$

2.3. Actions on Graßmannians. Let $H$ be a complex vector space, $r$ its dimension, $0<s<r, q=r-s$, and $\mathfrak{S r}^{q}(H)$ the Graßmann variety of $q$-dimensional quotient spaces of $H$. The special linear group $\operatorname{SL}(H)$ is acting in a natural way on $\mathfrak{G r}^{q}(H)$. Fix $(\chi: H \longrightarrow Q) \in \mathfrak{G r}^{q}(H)$. Suppose that $\lambda: \mathbb{C}^{\star} \longrightarrow \mathrm{SL}(H)$ is a one parameter subgroup and let $\left(H_{\bullet}, \varepsilon_{\bullet}\right)$ be the associated $\mathbb{Z}$-weighted flag. It is well-known and easy to see (compare [30], Chapter 4, §4, [31], Chapter 4, §6, [35], Exercise 1.5.1.15) that

$$
\mu(\lambda, \chi)=-\sum_{j=1}^{m+1} \varepsilon_{j} \cdot\left(\operatorname{dim}_{\mathbb{C}}\left(\chi\left(H_{j}\right)\right)-\operatorname{dim}_{\mathbb{C}}\left(\chi\left(H_{j-1}\right)\right)\right) .
$$

2.4. Linearized line bundles on quot schemes. Linearized line bundles on quot schemes for vector bundles on curves were studied by Drezet and Narasimhan [10] in characteristic zero. They correspond to line bundles on the moduli stack of vector bundles on $X$. The Picard groups of moduli stacks for principal $G$-bundles of fixed topological type $d \in \pi_{1}(X)$, $G$ a reductive linear algebraic group, were determined by Biswas and Hoffmann [3], building on a large selection of previously studied special cases.

Fix integers $r>0, d$, and $n>0$. Set $p_{0}:=d+r \cdot(1-g), p:=r \cdot n+p_{0}$, and pick a complex vector space $V$ of dimension $p$. We look at the quasi-projective quot scheme $\mathfrak{Q}$ that parameterizes quotients $\kappa: V \otimes \mathcal{O}_{X}(-n) \longrightarrow E$, such that $E$ is a vector bundle of rank $r$ and degree $d$ and $H^{0}(\kappa(n)): V \longrightarrow H^{0}(X, E(n))$ is an isomorphism. ${ }^{6}$ The group GL(V) acts on $\mathfrak{Q}$ via

$$
\begin{aligned}
\sigma: \mathrm{GL}(V) \times \mathfrak{Q} & \longrightarrow \mathfrak{Q} \\
(g, \kappa) & \longmapsto \kappa \circ\left(g^{-1} \otimes \operatorname{id}_{\mathcal{O}_{X}(-n)}\right) .
\end{aligned}
$$

Suppose $\mathscr{L}$ is a line bundle which is linearized with respect to the group action $\sigma$. Since the subgroup $\mathscr{Z}:=\mathbb{C}^{\star} \cdot \mathrm{id}_{V} \subset \mathrm{GL}(V)$ acts trivially on $\mathfrak{Q}$, there exists an integer $w$, such that $\mathscr{Z}$ acts via $\left(t \cdot \operatorname{id}_{V}, \ell\right) \longmapsto t^{w} \cdot \ell$ on each fiber of $\mathscr{L}$ over $\mathfrak{Q}$. We call $w$ the weight of $\mathscr{L}$.

We assume that, for every semistable vector bundle $E$ of rank $r$ and degree $d$ on $X$, $H^{1}(X, E(n))=\{0\}$. This implies that every semistable vector bundle $E$ of rank $r$ and degree $d$ can be written as a quotient $\kappa: V \otimes \mathcal{O}_{X}(-n) \longrightarrow E$, such that $H^{0}(\kappa(n)): V \longrightarrow$ $H^{0}(X, E(n))$ is an isomorphism.

Remark 2.4.1. i) We let $\mathfrak{Q}^{\text {ss }} \subset \mathfrak{Q}$ be the $\mathrm{GL}(V)$-invariant open subset consisting of quotients $\kappa: V \otimes \mathcal{O}_{X}(-n) \longrightarrow E$, such that $E$ is semistable. The complement of $\mathfrak{Q}^{\text {ss }}$ has codimension at least two ([22], proof of Corollary 3.2, p. 1311, [13], Theorem 8 (a), p. 53, compare also [4], Lemma 2.1). So, the Picard group of $G L(V)$-linearized line bundles on $\mathfrak{Q}$ agrees with the Picard group of $\mathrm{GL}(V)$-linearized line bundles on $\mathfrak{Q}^{\text {ss }}$.

${ }^{5}$ Since all pairs consisting of a maximal torus of $\mathrm{SL}_{r}(\mathbb{C})$ and a Borel subgroup containing that maximal torus are conjugate, this set does not depend on the choices of $S$ and $A$.

${ }^{6}$ This implies that $H^{1}(X, E(n))=\{0\}$. So, by [28], Theorem 8.2.1 (ii), $\mathfrak{Q}$ is smooth. The variety $\mathfrak{Q}$ is connected and has dimension $p^{2}+r^{2} \cdot(g-1)$. 
ii) Note that the stack quotient of $\mathfrak{Q}^{\text {ss }}$ by the action of $\operatorname{GL}(V)$ is the moduli stack $\operatorname{Bun}_{r / d}^{\text {ss }}(X)$ of semistable vector bundles of rank $r$ and degree $d$ on $X$. Therefore, the Picard group of $\mathrm{GL}(V)$-linearized line bundles on $\mathfrak{Q}^{\text {ss }}$ agrees with the Picard group of the stack $\operatorname{Bun}_{r / d}^{\text {ss }}(X)$ ([39], Tag 06WT). As recalled in Part i), the complement of $\operatorname{Bun}_{r / d}^{\text {ss }}(X)$ in the moduli stack $\operatorname{Bun}_{r / d}(X)$ of all vector bundles of rank $r$ and degree $d$ on $X$ has codimension at least two, so that we may identify the Picard groups of $\operatorname{Bun}_{r / d}^{\text {ss }}(X)$ and $\operatorname{Bun}_{r / d}(X)$. Altogether, we see that the Picard group of GL(V)-linearized line bundles on $\mathfrak{Q}$ agrees with the Picard group of $\operatorname{Bun}_{r / d}(X)$.

Let $E_{\mathfrak{B}}$ be the tautological vector bundle on the stack $\operatorname{Bun}_{r / d}(X) \times X$ and $\kappa_{\mathfrak{Q}}: V \otimes$ $\pi_{X}^{\star}\left(\mathcal{O}_{X}(-n)\right) \longrightarrow E_{\mathfrak{Q}}$ the universal quotient on $\mathfrak{Q} \times X$.

Remark 2.4.2. Clearly, the vector bundle $E_{\mathfrak{Q}}$ is the pullback of $E_{\mathfrak{B}}$ under the morphism $\mathfrak{Q} \times X \longrightarrow \operatorname{Bun}_{r / d}(X) \times X$.

Example 2.4.3. i) Let $\mathfrak{A}$ be a scheme and $\mathscr{F}$ a coherent $\mathcal{O}_{\mathfrak{A} \times X}$-module which is flat over $\mathfrak{A}$. Let us explain how the determinant of cohomology of $\mathscr{F}$ is constructed. The general formalism has been developed in [25]. We use the construction presented in [23], Section 2.1, and [38], Section 6.1. For large $k$, the direct image $\pi_{\mathscr{A} \star}\left(\mathscr{F} \otimes \pi_{X}^{\star}\left(\mathcal{O}_{X}(k)\right)\right)$ will be locally free. We set

$$
P_{0}:=\pi_{\mathfrak{A}}^{\star}\left(\pi_{\mathfrak{A} \star}\left(\mathscr{F} \otimes \pi_{X}^{\star}\left(\mathcal{O}_{X}(k)\right)\right)\right) \otimes \pi_{X}^{\star}\left(\mathcal{O}_{X}(-k)\right) .
$$

Note that we have a surjection

$$
P_{0} \longrightarrow \mathscr{F}
$$

and that $\pi_{\mathfrak{A} \star}\left(P_{0}\right)=0$. We let $P_{1}$ be the kernel of the above surjection. By Hilbert's syzygy theorem and [23], Lemma 2.1.7, $P_{1}$ is also locally free and satisfies $\pi_{\mathscr{A} \star}\left(P_{1}\right)=0$. Then, the complex

$$
R^{1} \pi_{\mathfrak{A} \star}\left(P_{1}\right) \longrightarrow R^{1} \pi_{\mathfrak{A} \star}\left(P_{0}\right)
$$

of locally free sheaves on $\mathfrak{A}$ has the direct image sheaves of $\mathscr{F}$ as cohomology sheaves, and this construction is compatible with base change. We define the determinant of cohomology of $\mathscr{F}$ as

$$
D(\mathscr{F}):=\operatorname{det}\left(R^{1} \pi_{\mathscr{A} \star}\left(P_{0}\right)\right) \otimes \operatorname{det}\left(R^{1} \pi_{\mathscr{A} \star}\left(P_{1}\right)\right)^{\vee} .
$$

This construction has the following properties (compare [12], Section 2, [29], Section 1):

- (Base change.) For a morphism $f: \mathfrak{A}^{\prime} \longrightarrow \mathfrak{A}$ and an $\mathfrak{A}$-flat coherent $\mathcal{O}_{\mathfrak{A} \times X}$-module $\mathscr{F}$, one has

$$
D\left(\left(f \times \operatorname{id}_{X}\right)^{\star}(\mathscr{F})\right)=f^{\star}(D(\mathscr{F})) .
$$

- (Short exact sequences.) Let

$$
0 \longrightarrow \mathscr{F}_{1} \longrightarrow \mathscr{F}_{2} \longrightarrow \mathscr{F}_{3} \longrightarrow 0
$$

be a short exact sequence of $\mathfrak{A}$-flat coherent $\mathcal{O}_{\mathfrak{A} \times X}$-modules. Then,

$$
D\left(\mathscr{F}_{2}\right) \cong D\left(\mathscr{F}_{1}\right) \otimes D\left(\mathscr{F}_{3}\right) \text {. }
$$

- (Projection formula.) For an $\mathfrak{A}$-flat coherent $\mathcal{O}_{\mathfrak{A} \times X}$-module $\mathscr{F}$ and an invertible $\mathcal{O}_{\mathfrak{A}}$-module $\mathscr{L}$,

$$
D\left(\mathscr{F} \otimes \pi_{\mathfrak{A}}^{\star}(\mathscr{L})\right) \cong D(\mathscr{F}) \otimes \mathscr{L}^{\otimes \chi} .
$$

Here, $\chi$ is the Euler characteristic of the restricted sheaf $\mathscr{F}_{\mid\{s\} \times X}, s \in S$. 
Using this, we define the line bundle $D_{\mathfrak{Q}}$ on $\mathfrak{Q}$ as the determinant of cohomology of the universal family $E_{\mathfrak{Q}}$. The techniques apply also to the universal bundle $E_{\mathfrak{B}}$ on $\operatorname{Bun}_{r / d}(X) \times$ $X$ and lead to the line bundle $D_{\mathfrak{B}}$ on $\operatorname{Bun}_{r / d}(X)$. By the functorial properties of the determinant of cohomology, $D_{\mathfrak{Q}}$ is isomorphic to the pullback of $D_{\mathfrak{B}}$ under the morphism $\mathfrak{Q} \longrightarrow \operatorname{Bun}_{r / d}(X)$. The line bundle $D_{\mathbb{Q}}$ is linearized with respect to the $\mathrm{GL}(V)$-action on $\mathfrak{Q}$, and its weight is $p_{0}=d+r \cdot(1-g)$. Faltings used the determinant of cohomology in his alternative construction of the moduli spaces of vector and Higgs bundles on curves ([14], see also [20]).

ii) We may view $E_{\mathfrak{B} \mid \operatorname{Bun}_{r / d}(X) \times\left\{x_{0}\right\}}$ as a vector bundle on $\operatorname{Bun}_{r / d}(X)$. Let $R_{\mathfrak{B}}$ be the determinant of this vector bundle. Similarly, we define the line bundle $R_{\mathfrak{Q}}$ on $\mathfrak{Q}$. It is linearized with respect to the $\mathrm{GL}(V)$-action on $\mathfrak{Q}$, its weight is $r$, and it is isomorphic to the pullback of $R_{\mathfrak{B}}$ under the morphism $\mathfrak{Q} \longrightarrow \mathrm{Bun}_{r / d}(X)$.

We need the natural morphism

$$
\begin{aligned}
\mathfrak{D}_{\mathfrak{Q}}: \mathfrak{Q} & \longrightarrow \operatorname{Pic}^{d}(X) \\
\left(\kappa: V \otimes \mathscr{O}_{X}(-n) \longrightarrow E\right) & \longmapsto \operatorname{det}(E) .
\end{aligned}
$$

It factorizes over the morphism $\mathfrak{Q} \longrightarrow \operatorname{Bun}_{r / d}(X)$.

Proposition 2.4.4. The quotient of the Picard group of $\operatorname{Bun}_{r / d}(X)$ by the Picard group $\operatorname{Pic}^{d}(X)$ is freely generated by $D_{\mathfrak{B}}$ and $R_{\mathfrak{B}}$.

Proof. This is contained in [3], Theorem 5.3.1, iii).

Let's do some computations with the linearized line bundles we have just constructed.

Lemma 2.4.5. i) For $k \in \mathbb{N}$, the determinant of cohomology of the vector bundle $E_{\mathfrak{Q}}(k):=$ $E_{\mathfrak{Q}} \otimes \pi_{X}^{\star}\left(\mathcal{O}_{X}(k)\right)$ is isomorphic to $D_{\mathbb{Q}} \otimes R_{\mathfrak{Q}}^{\otimes k}$.

ii) The determinant of cohomology of $E_{\mathbb{Q}}(n)$ is isomorphic to the trivial line bundle linearized by the character det: $\mathrm{GL}(V) \longrightarrow \mathbb{C}^{\star}$.

Proof. i) We prove the result by induction on $k$, the case $k=0$ being trivial. The short exact sequence

$$
\{0\} \longrightarrow \mathcal{O}_{X} \longrightarrow \mathcal{O}_{X}(1) \longrightarrow \mathcal{O}_{\left\{x_{0}\right\}} \longrightarrow\{0\}
$$

may be pulled back to $\mathfrak{Q} \times X$ and tensorized by $E_{\mathfrak{Q}}(k)$. The result is the short exact sequence

$$
\{0\} \longrightarrow E_{\mathfrak{Q}}(k) \longrightarrow E_{\mathfrak{Q}}(k+1) \longrightarrow E_{\mathfrak{Q} \mid \mathfrak{Q} \times\left\{x_{0}\right\}} \longrightarrow\{0\} .
$$

The determinant of cohomology of $E_{\mathfrak{Q} \mid \mathbb{Q} \times\left\{x_{0}\right\}}$ is $R_{\mathfrak{Q}}$. So, the claim follows from the induction hypothesis and the formula for the determinant of cohomology of a short exact sequence.

ii) By our general assumptions, the push forward of the quotient homomorphism $V \otimes$ $\mathcal{O}_{\mathbb{Q} \times X} \longrightarrow E_{\mathbb{Q}}(n)$ is an isomorphism $V \otimes \mathcal{O}_{\mathbb{Q}} \longrightarrow \pi_{\mathbb{Q} \star}\left(E_{\mathbb{Q}}(n)\right)$, and the higher direct images of $E_{\mathfrak{Q}}(n)$ are zero. So, the determinant of cohomology of $E_{\mathfrak{Q}}(n)$ is trivial, and the result follows from Part i).

In constructions with geometric invariant theory, we need linearized line bundles of weight zero in order to have any hope for finding semistable points. Let $\mathscr{L}$ be a GL $(V)$ linearized line bundle of weight $w$. Denote by $\mathscr{L}_{0}$ the trivial line bundle linearized by the character det: $\mathrm{GL}(V) \longrightarrow \mathbb{C}^{\star}$. It has weight $p$. So,

$$
\mathscr{L}^{\otimes p} \otimes \mathscr{L}_{0}^{\otimes-w}
$$


is a $\mathrm{GL}(V)$-linearized line bundle of weight zero. In the following computations, we will write such a line bundle in additive notation as

$$
p \cdot \mathscr{L}-w \cdot \mathscr{L}_{0} .
$$

Pick $m \in \mathbb{N}$ and let $\mathscr{D}_{m}$ be the determinant of cohomology of $E_{\mathbb{Q}}(m+n)$. By Lemma 2.4.5, we have

$$
\begin{aligned}
& p \cdot \mathscr{D}_{m}-w \cdot \mathscr{L}_{0} \\
= & \left(p_{0}+n \cdot r\right) \cdot\left(D_{\mathfrak{Q}}+(m+n) \cdot R_{\mathfrak{Q}}\right)-\left(p_{0}+(m+n) \cdot r\right) \cdot\left(D_{\mathfrak{Q}}+n \cdot R_{\mathfrak{Q}}\right) \\
= & m \cdot\left(-r \cdot D_{\mathfrak{Q}}+p_{0} \cdot R_{\mathfrak{Q}}\right) .
\end{aligned}
$$

Set $u_{0}:=a \cdot r^{a-1} \cdot d+r^{a} \cdot(1-g)$ and $u:=a \cdot r^{a} \cdot n+u_{0}$. The determinant $T_{\mathfrak{B}}$ of cohomology of $E_{\mathfrak{B}}^{\otimes a}$ is a line bundle on $\operatorname{Bun}_{r / d}(X)$. Its weight is $a \cdot u_{0} \cdot{ }^{7}$ Note that it is the pullback of the determinant of cohomology of the tautological vector bundle on $\operatorname{Bun}_{r^{a} / a \cdot r^{a-1} \cdot d}(X)$ via the morphism $\operatorname{Bun}_{r / d}(X) \longrightarrow \operatorname{Bun}_{r^{a} / a \cdot r^{a-1} \cdot d}(X)$ induced by the representation $\varrho: \mathrm{GL}_{r}(\mathbb{C}) \longrightarrow \mathrm{GL}\left(W^{\otimes a}\right), W:=\mathbb{C}^{r}$.

Proposition 2.4.6. Up to a possible twist by the pullback of a line bundle on $\mathrm{Pic}^{d}(X)$,

$$
T_{\mathfrak{B}}=a \cdot r^{a-1} \cdot D_{\mathfrak{B}}+y \cdot R_{\mathfrak{B}},
$$

for an appropriate integer $y$.

Remark 2.4.7. Of course, $y$ may be computed from the weight of $T_{\mathfrak{B}}$. We will not need the explicit form of $y$ in the sequel.

First proof of Proposition 2.4.6. We write

$$
T_{\mathfrak{B}}=x \cdot D_{\mathfrak{B}}+y \cdot R_{\mathfrak{B}} .
$$

It follows from a result of Kumar, Narasimhan, and Ramanathan ([26], Theorem (5.4)) and Biswas and Hoffmann ([3], Proposition 4.4.4) that $x$ is the Dynkin index ([3], Remark 4.3.3,

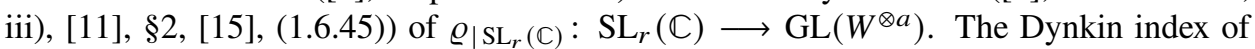
the standard representation $\mathrm{SL}_{r}(\mathbb{C}) \longrightarrow \mathrm{GL}(W)$ is one ([26], Lemma (5.2)), and for tensor products of representations, the formula

$$
\operatorname{Dyn}\left(\tau_{1} \otimes \tau_{2}\right)=\operatorname{dim}_{\mathbb{C}}\left(\tau_{2}\right) \cdot \operatorname{Dyn}\left(\tau_{1}\right)+\operatorname{dim}_{\mathbb{C}}\left(\tau_{1}\right) \cdot \operatorname{Dyn}\left(\tau_{2}\right)
$$

holds true ([15], (1.6.47)). This shows that the Dynkin index of $\varrho_{\mid \mathrm{SL}_{r}(\mathbb{C})}$ is $a \cdot r^{a-1}$.

Second proof of Proposition 2.4.6. Here, we will work with linearized sheaves on the quot scheme $\mathfrak{Q}$. Let $\mathfrak{D}_{\mathfrak{Q}}$ be the determinant morphism from (7). Since we would like to perform our computations modulo the Picard group $\operatorname{Pic}^{d}(X)$ and forming the determinant in cohomology commutes with base change, we may restrict to a fiber of $\mathfrak{D}_{\mathfrak{Q}}$. So, let $L$ be a line bundle of degree $d$ on $X$ and $\mathfrak{Q}_{L}$ be the quot scheme, parameterizing quotients $\kappa: V \otimes \mathcal{O}_{X}(-n) \longrightarrow E$ with $\operatorname{det}(E) \cong L$. Then, there is a line bundle $N$ on $\mathfrak{Q}_{L}$, such that

$$
\operatorname{det}\left(E_{\mathbb{Q}_{L}}\right) \cong \pi_{\mathbb{Q}_{L}}^{\star}(N) \otimes \pi_{X}^{\star}(L),
$$

i.e.,

$$
c_{1}\left(E_{\mathfrak{Q}_{L}}\right)=c_{1}\left(\pi_{\mathfrak{Q}_{L}}^{\star}(N)\right)+c_{1}\left(\pi_{X}^{\star}(L)\right) .
$$

${ }^{7}$ The weight of the determinant of cohomology is the rank of the determinant of cohomology, $u_{0}$ in this case, multiplied by the weight of the sheaf, which is $a$ in the case at hand. 
The Grothendieck-Riemann-Roch theorem ([16], Theorem 15.2, [19], Appendix A, Theorem 5.3) states that

$$
\operatorname{ch}\left(\pi_{\mathfrak{Q}_{L} !}\left(E_{\mathfrak{Q}_{L}}\right)\right)=\pi_{\mathfrak{Q}_{L} \star}\left(\operatorname{ch}\left(E_{\mathbb{Q}_{L}}\right) \cdot \operatorname{td}\left(T_{\pi_{\mathfrak{Q}_{L}}}\right)\right) .
$$

The degree one component is the first Chern class of $D_{\mathfrak{Q}_{L}}$. Since the relative dimension of $\pi_{\mathfrak{Q}_{L}}$ is one, we need to look at the degree two component of the class in the brackets of the right hand side of the equation. Now, setting $c:=c_{1}\left(\pi_{X}^{\star}\left(T_{X}\right)\right)$, we have

$$
\operatorname{td}\left(T_{\pi_{\Omega_{L}}}\right)=\pi_{X}^{\star}(\operatorname{td}(X))=1+\frac{1}{2} \cdot c .
$$

Up to algebraic equivalence, we have

$$
\frac{1}{2} \cdot c=(1-g) \cdot\left[\mathfrak{Q}_{L} \times\left\{x_{0}\right\}\right] .
$$

The degree two component we need to look at is

$$
(1-g) \cdot c_{1}\left(E_{\mathbb{Q}_{L}}\right) \cdot\left[\mathfrak{Q}_{L} \times\left\{x_{0}\right\}\right]+\operatorname{ch}_{2}\left(E_{\mathbb{Q}_{L}}\right) .
$$

Here, we would like to point out that $\pi_{X}^{\star}(L)$. $\left[\mathfrak{Q}_{L} \times\left\{x_{0}\right\}\right]=0$, so that

$$
c_{1}\left(\pi_{\mathfrak{Q}_{L}}^{\star}(N)\right) \cdot\left[\mathfrak{Q}_{L} \times\left\{x_{0}\right\}\right]=c_{1}\left(E_{\mathfrak{Q}_{L}}\right) \cdot\left[\mathfrak{Q}_{L} \times\left\{x_{0}\right\}\right] .
$$

Let

$$
\begin{aligned}
\iota_{0}: \mathfrak{Q}_{L} & \longrightarrow \mathfrak{Q}_{L} \times X \\
\kappa & \longmapsto\left(\kappa, x_{0}\right)
\end{aligned}
$$

be the inclusion. By definition,

$$
\iota_{0}^{\star}\left(\operatorname{det}\left(E_{\mathbb{Q}_{L}}\right)\right)=R_{\mathfrak{Q}_{L}} .
$$

So, the projection formula ([16], Proposition 2.5 (c)) yields

$$
c_{1}\left(E_{\mathfrak{Q}_{L}}\right) \cdot\left[\mathfrak{Q}_{L} \times\left\{x_{0}\right\}\right]=\iota_{0 \star}\left(c_{1}\left(R_{\mathfrak{Q}_{L}}\right)\right) .
$$

Since $\pi_{\mathfrak{Q}_{L}} \circ \iota_{0}=\mathrm{id}_{\mathfrak{Q}_{L}}$, we see that

$$
c_{1}\left(D_{\mathfrak{Q}_{L}}\right)=(1-g) \cdot c_{1}\left(R_{\mathfrak{Q}_{L}}\right)+\pi_{\mathfrak{Q}_{L} \star}\left(\operatorname{ch}_{2}\left(E_{\mathfrak{Q}_{L}}\right)\right) .
$$

Now, in order to prove the proposition, we need to carry out the analogous computation for $E_{\mathbb{Q}_{L}}^{\otimes a}$. Since

$$
\operatorname{ch}\left(E_{\mathfrak{Q}_{L}}^{\otimes a}\right)=\operatorname{ch}\left(E_{\mathfrak{Q}_{L}}\right)^{a},
$$

we see that

$$
\begin{aligned}
c_{1}\left(E_{\mathfrak{Q}_{L}}^{\otimes a}\right) & =a \cdot r^{a-1} \cdot c_{1}\left(E_{\mathfrak{Q}_{L}}\right), \\
\operatorname{ch}_{2}\left(E_{\mathfrak{Q}_{L}}^{\otimes a}\right) & =\left(\begin{array}{l}
a \\
2
\end{array}\right) \cdot r^{a-2} \cdot c_{1}\left(E_{\mathfrak{Q}_{L}}\right)^{2}+a \cdot r^{a-1} \cdot \operatorname{ch}_{2}\left(E_{\mathfrak{Q}_{L}}\right) .
\end{aligned}
$$

Up to algebraic equivalence, we have

$$
c_{1}\left(\pi_{X}^{\star}(L)\right)=d \cdot\left[\mathfrak{Q}_{L} \times\left\{x_{0}\right\}\right] .
$$

Invoking (10), we compute

$$
\begin{aligned}
c_{1}\left(E_{\mathfrak{Q}_{L}}\right)^{2} & =c_{1}\left(\pi_{\mathfrak{Q}_{L}}^{\star}(N)\right)^{2}+2 d \cdot c_{1}\left(E_{\mathfrak{Q}_{L}}\right) \cdot\left[\mathfrak{Q}_{L} \times\left\{x_{0}\right\}\right] \\
& =\pi_{\mathfrak{Q}_{L}}^{\star}\left(c_{1}(N)^{2}\right)+2 d \cdot c_{1}\left(E_{\mathfrak{Q}_{L}}\right) \cdot\left[\mathfrak{Q}_{L} \times\left\{x_{0}\right\}\right] .
\end{aligned}
$$


Since the relative dimension of $\pi_{\mathfrak{Q}_{L}}$ is one, the projection formula ([16], Proposition 8.3 (c)) shows that $\pi_{\mathfrak{Q}_{L} \star}\left(\pi_{\mathfrak{Q}_{L}}^{\star}(\beta)\right)=0$, for every $\beta \in A^{\star}\left(\mathfrak{Q}_{L}\right)$. Using this observation, we arrive at the formula

$c_{1}\left(T_{\mathfrak{Q}_{L}}\right)=\left((1-g) \cdot a \cdot r^{a-1}+2 \cdot d \cdot\left(\begin{array}{l}a \\ 2\end{array}\right) \cdot r^{a-2}\right) \cdot c_{1}\left(R_{\mathfrak{Q}_{L}}\right)+a \cdot r^{a-1} \cdot \pi_{\mathfrak{Q}_{L} \star}\left(\operatorname{ch}_{2}\left(E_{\mathfrak{Q}_{L}}\right)\right)$.

A comparison of this formula with (11) shows that the assertion of the proposition is true.

Lemma 2.4.8. Let $k \in \mathbb{N}$ and $E_{\mathfrak{Q}}(k):=E_{\mathfrak{Q}} \otimes \pi_{X}^{\star}\left(\mathcal{O}_{X}(k)\right)$. Then, the determinant of cohomology of $E_{\mathbb{Q}}(k)^{\otimes a}$ is isomorphic to $T_{\mathbb{Q}} \otimes R_{\mathfrak{Q}}^{\otimes\left(a^{2} \cdot r^{a-1} \cdot k\right)}$.

Proof. For $l \in \mathbb{Z}$, we set $F_{\mathfrak{Q}}(l):=E_{\mathbb{Q}}^{\otimes a} \otimes \pi_{X}^{\star}\left(\mathcal{O}_{X}(l)\right)$, so that $E_{\mathbb{Q}}(k)^{\otimes a} \cong F_{\mathbb{Q}}(a \cdot k)$, $k \in \mathbb{Z}$. We prove the claim by induction on $k$. For $k=0$, there is nothing to prove. For the inductive step, we use the short exact sequence

$\{0\} \longrightarrow F_{\mathfrak{Q}}(l) \longrightarrow F_{\mathfrak{Q}}(l+1) \longrightarrow\left(E_{\mathfrak{Q}}^{\otimes a}\right)_{\mid \mathbb{Q} \times\left\{x_{0}\right\}} \longrightarrow\{0\}$.

It shows that the determinant of cohomology of $F_{\mathbb{Q}}(l+1)$ differs from the one of $F_{\mathfrak{Q}}(l)$ by the factor

$$
\operatorname{det}\left(\left(E_{\mathbb{Q}}^{\otimes a}\right)_{\mid \mathfrak{Q} \times\left\{x_{0}\right\}}\right) \cong R_{\mathfrak{Q}}^{\otimes a \cdot r^{a-1}} .
$$

This enables us to reduce the case $k+1$ to the case $k$.

Let $\mathscr{L}$ be the determinant of cohomology of $E_{\mathbb{Q}}(n)^{\otimes a}$. It is a $\mathrm{GL}(V)$-linearized line bundle of weight $w:=a^{2} \cdot r^{a} \cdot n+a \cdot u_{0}$. Using additive notation again, the line bundle $p \cdot \mathscr{L}-w \cdot \mathscr{L}_{0}$ has weight zero. With Lemma 2.4.8, we compute

$$
\begin{aligned}
& p \cdot \mathscr{L}-w \cdot \mathscr{L}_{0} \\
= & \left(r n+p_{0}\right) \cdot\left(x D_{\mathfrak{Q}}+\left(a^{2} r^{a-1} n+y\right) R_{\mathfrak{Q}}\right)-\left(x p_{0}+y r+a^{2} r^{a} n\right) \cdot\left(D_{\mathfrak{Q}}+n R_{\mathfrak{Q}}\right) \\
= & \left(\left(a^{2} \cdot r^{a-1}-x\right) \cdot n+y\right) \cdot\left(p_{0} \cdot R_{\mathfrak{Q}}-r \cdot D_{\mathfrak{Q}}\right) .
\end{aligned}
$$

After plugging in the value for $x$ found in Lemma 2.4.8, this reads

$$
p \cdot \mathscr{L}-w \cdot \mathscr{L}_{0}=\left(\left(a^{2} \cdot r^{a-1}-a \cdot r^{a-1}\right) \cdot n+y\right) \cdot\left(p_{0} \cdot R_{\mathfrak{Q}}-r \cdot D_{\mathfrak{Q}}\right) .
$$

\section{Moduli SPACES}

In this part, we will look at the moduli problem for coherent $\varrho$-systems for the representation $\varrho$ of $\mathrm{GL}_{r}(\mathbb{C})$ on $\mathrm{GL}\left(W^{\otimes a}\right), W:=\mathbb{C}^{r}$, given as the $a$-fold tensor power of the standard representation, for $a \geq 2$. We also assume $L=\mathscr{O}_{X}$. This should be a key step for understanding the moduli problems for arbitrary homogeneous representations. We will carry out the construction with the help of geometric invariant theory. One important step is to rewrite the quantity $\mu_{(E, \Gamma)}\left(E_{\bullet}, \alpha_{\bullet}\right)$ in such a way that the computations of [24] and [35] may be adapted. In this context, it is also crucial to find the right linearization of the group action. Here, we will see that the line bundle in which we will linearize the group action will be ample only for stability parameters below some threshold (14). Throughout this section, we fix the type $\left(\mathcal{O}_{X}, d, s\right)$ and the stability parameter $\delta$. 
3.1. Boundedness. In order to check that the family of all vector bundles $E$ for which there exists a subspace $\Gamma \subset H^{0}\left(X, E^{\otimes a}\right)$, such that $(E, \Gamma)$ is a $\delta$-semistable coherent $\varrho$-system of type $\left(\mathcal{O}_{X}, d, s\right)$ is bounded, we have to find a constant $C_{0}$, such that, for every $\delta$-semistable coherent $\varrho$-system $(E, \Gamma)$ of type $\left(\mathscr{O}_{X}, d, s\right)$ and every subbundle $\{0\} \subsetneq F \subsetneq E$, the inequality

$$
\mu(F) \leq \frac{r}{r}+C_{0}
$$

is satisfied. So, assume that $(E, \Gamma)$ is a $\delta$-semistable coherent $\varrho$-system of type $\left(\mathcal{O}_{X}, d, s\right)$ and $\{0\} \subsetneq F \subsetneq E$ is a subbundle of $E$. Let $\left(B_{\bullet}:\{0\} \subsetneq B_{1} \subsetneq \cdots \subsetneq B_{m+1} \subsetneq E^{\otimes a}, \beta_{\bullet}=\right.$ $\left.\left(\beta_{1}, \ldots, \beta_{m+1}\right)\right)$ be the $\mathbb{Q}_{>0}$-weighted filtration associated with the $\mathbb{Q}_{>0}$-weighted filtration $(\{0\} \subsetneq F \subsetneq E,(1))$ of $E$. As before, we let $\varepsilon_{\bullet}=\left(\varepsilon_{1}, \ldots, \varepsilon_{m+1}\right)$ be the vector of $\mathbb{Z}$-weights. The values for $\varepsilon_{1}, \ldots, \varepsilon_{m+1}$ are

$$
a \cdot \operatorname{rk}(F)-b \cdot r, \quad b=0, \ldots, a,
$$

i.e.,

$$
m=a \quad \text { and } \quad \varepsilon_{j}=a \cdot(\operatorname{rk}(F)-r)+(j-1) \cdot r, \quad j=1, \ldots, a+1 .
$$

So,

$$
\beta_{j}=\frac{1}{r^{a-1}}, \quad j=1, \ldots, a
$$

Clearly,

$$
\operatorname{rk}\left(B_{j}\right) \leq r^{a}-1, \quad j=1, \ldots, a .
$$

We conclude

$$
\mu_{(E, \Gamma)}(\{0\} \subsetneq F \subsetneq E,(1)) \leq a \cdot r \cdot s .
$$

By $\delta$-semistability,

$$
\mu(F) \leq \frac{d}{r}+\delta \cdot \frac{\mu_{(E, \Gamma)}(\{0\} \subsetneq F \subsetneq E,(1))}{\operatorname{rk}(F) \cdot r} \leq \frac{d}{r}+\delta \cdot a \cdot s .
$$

3.2. A parameter space. Suppose that we are given a bounded family $\mathfrak{B}$ of vector bundles on $X$. We assume that $E$, $\operatorname{det}(E) \in \mathfrak{B}$, for every $\delta$-semistable coherent $\varrho$-system $(E, \Gamma)$ of type $\left(\mathcal{O}_{X}, d, s\right)$.

We may find an index $n_{0} \in \mathbb{N}$, such that, for all $n \geq n_{0}$, the following properties are satisfied:

- For every vector bundle $E \in \mathfrak{B}$, the vector bundle $E(n)$ is globally generated and $H^{1}(X, E(n))=\{0\}$.

- For vector bundles $F_{1}, \ldots, F_{a} \in \mathfrak{B}, F_{1}(n) \otimes \cdots \otimes F_{a}(n)$ is globally generated, $H^{1}\left(X, F_{1}(n) \otimes \cdots \otimes F_{a}(n)\right)=\{0\}$, and the natural linear map

$$
H^{0}\left(X, F_{1}(n)\right) \otimes \cdots \otimes H^{0}\left(X, F_{a}(n)\right) \longrightarrow H^{0}\left(X, F_{1}(n) \otimes \cdots \otimes F_{a}(n)\right)
$$

is surjective. 
- For $f \leq r^{a},{ }^{8}$ a vector bundle $E \in \mathfrak{B}$, subbundles $F_{i}^{j}, i=1, \ldots, a, j=1, \ldots, f$, of $E$ which also belong to $\mathfrak{B}$,

$$
\Sigma:=\sum_{j=1}^{f} F_{1}^{j}(n) \otimes \cdots \otimes F_{a}^{j}(n)
$$

is globally generated, $H^{1}(X, \Sigma)=\{0\}$, and the natural linear map

$$
\bigoplus_{j=1}^{f} H^{0}\left(X, F_{1}^{j}(n) \otimes \cdots \otimes F_{a}^{j}(n)\right) \longrightarrow H^{0}(X, \Sigma)
$$

is surjective. (The sum is taken inside $E(n)^{\otimes a}$.)

Fix $n \geq n_{0}$, set $p:=r(n+1-g)+d, p(a):=r^{a} \cdot(a \cdot n+1-g)+a \cdot r^{a-1} \cdot d$, $q:=p(a)-s$, pick a vector space $V$ of dimension $p$, let $\mathfrak{Q}$ be the quasi-projective quot scheme parameterizing quotients $\kappa: V \otimes \mathcal{O}_{X}(-n) \longrightarrow E$ where $E$ is a vector bundle on $X$ of rank $r$ and degree $d$ and $H^{0}(X, \kappa(n)): V \longrightarrow H^{0}(X, E(n))$ is an isomorphism, and $\mathfrak{G}$ the Graßmannian of $q$-dimensional quotients of $V^{\otimes a}$.

On $\mathfrak{I}:=\mathfrak{Q} \times \mathfrak{G}$, there is the universal quotient

$$
\chi_{\mathfrak{I}}: V^{\otimes a} \otimes \mathcal{O}_{\mathfrak{I}} \longrightarrow K_{\mathfrak{I}},
$$

obtained by pulling back the universal quotient $\chi_{\mathfrak{G}}: V^{\otimes a} \otimes \mathcal{O}_{\mathfrak{G}} \longrightarrow K_{\mathfrak{G}}$ on $\mathfrak{G}$ via $\pi_{\mathfrak{G}}$, and, on $\mathfrak{T} \times X$, there is the universal quotient

$$
\kappa \mathfrak{T}: V \otimes \pi_{X}^{\star}\left(\mathcal{O}_{X}(-n)\right) \longrightarrow E_{\mathfrak{I}},
$$

obtained by pulling back the universal quotient $\kappa_{\mathfrak{Q}}: V \otimes \pi_{X}^{\star}\left(\mathcal{O}_{X}(-n)\right) \longrightarrow E_{\mathfrak{Q}}$ on $\mathfrak{Q} \times X$ via $\pi_{\mathfrak{Q}} \times \operatorname{id}_{X}$. By the assumptions made at the beginning of this section, the sheaf

$$
H_{\varrho, \mathfrak{I}}:=\pi_{\mathfrak{I} \star}\left(\left(E_{\mathfrak{I}} \otimes \pi_{X}^{\star}\left(\mathcal{O}_{X}(n)\right)\right)^{\otimes a}\right)
$$

is locally free of rank $p(a)$. Our assumptions also imply that the homomorphism

$$
V^{\otimes a} \otimes \mathcal{O}_{\mathfrak{I}} \longrightarrow H_{\varrho, \mathfrak{I}}
$$

is surjective. Let $A_{\varrho, \mathfrak{I}}$ be the kernel of this homomorphism. The subscheme $\mathfrak{S}^{\prime}$ is defined as the locus where the homomorphism

$$
A_{\varrho, \mathfrak{I}} \longrightarrow K_{\mathfrak{I}}
$$

between locally free sheaves is zero.

Remark 3.2.1. On $\mathfrak{Q}$, we may define the vector bundle

$$
H_{\varrho, \mathfrak{Q}}:=\pi_{\mathfrak{Q} \star}\left(\left(E_{\mathfrak{Q}} \otimes \pi_{X}^{\star}\left(\mathcal{O}_{X}(n)\right)\right)^{\otimes a}\right)
$$

${ }^{8}$ Given a $\mathbb{Z}$-weighted filtration $\left(\{0\}=: E_{0} \subsetneq E_{1} \subsetneq \ldots \subsetneq E_{l} \subsetneq E_{l+1}:=E,\left(\gamma_{1}, \ldots, \gamma_{l+1}\right)\right)$ of a vector bundle $E$ of rank $r$, the weights occuring in the induced weighted filtration $\left(B_{\bullet}, \varepsilon_{\bullet}\right)$ of $E^{\otimes a}$ are of the form $\gamma_{i_{1}}+\cdots+\gamma_{i_{a}}$ with $\left(i_{1}, \ldots, i_{a}\right) \in\{1, \ldots, l+1\}^{\times a}$. So, for a weight $\varepsilon \in \mathbb{Z}$, we set

$$
I(\varepsilon):=\left\{\left(i_{1}, \ldots, i_{a}\right) \in\{1, \ldots, l+1\}^{\times a} \mid \gamma_{i_{1}}+\cdots+\gamma_{i_{a}} \leq \varepsilon\right\} .
$$

Then, the subbundle of weight $\varepsilon$ in the weighted filtration $\left(B_{\bullet}, \varepsilon_{\bullet}\right)$ is

$$
\sum_{\left(i_{1}, \ldots, i_{a}\right) \in I(\varepsilon)} E_{i_{1}} \otimes \cdots \otimes E_{i_{a}}
$$

the sum being taken inside $E^{\otimes a}$. Since $l+1 \leq r$, the cardinality of $I(\varepsilon)$ is at most $r^{a}$. 
The scheme $\mathfrak{S}^{\prime}$ that we have just constructed is the Graßmann bundle $\mathfrak{G r}^{q}\left(H_{\varrho}, \mathfrak{Q}\right)$ over $\mathfrak{Q}$. Since $\mathfrak{Q}$ is a smooth quasi-projective variety ([28], Theorem 8.2.1), we see that $\mathfrak{S}^{\prime}$ is a smooth quasi-projective variety, too. The above contruction shows how it is embedded into $\mathfrak{T}$, and this will help us to understand the group action and its linearizations.

Let $Z \subset X$ be the subscheme defined by the sheaf $\mathcal{O}_{X}(-a \cdot n)$. On $\mathfrak{T} \times X$, there is the short exact sequence

$$
\begin{aligned}
\{0\} \longrightarrow E_{\mathfrak{T}}^{\otimes a} & \longrightarrow\left(E_{\mathfrak{T}} \otimes \pi_{X}^{\star}\left(\mathcal{O}_{X}(n)\right)\right)^{\otimes a} \longrightarrow \\
& \longrightarrow\left(E_{\mathfrak{T}} \otimes \pi_{X}^{\star}\left(\mathcal{O}_{X}(n)\right)\right)^{\otimes a} \otimes \pi_{X}^{\star}\left(\mathcal{O}_{Z}\right) \longrightarrow\{0\} .
\end{aligned}
$$

The sheaf $\left(E_{\mathfrak{I}} \otimes \pi_{X}^{\star}\left(\mathcal{O}_{X}(n)\right)\right)^{\otimes a} \otimes \pi_{X}^{\star}\left(\mathcal{O}_{Z}\right)$ is flat over $\mathfrak{T}$, and the sheaf

$$
Q_{\varrho, \mathfrak{I}}:=\pi_{\mathfrak{I} \star}\left(\left(E_{\mathfrak{T}} \otimes \pi_{X}^{\star}\left(\mathcal{O}_{X}(n)\right)\right)^{\otimes a} \otimes \pi_{X}^{\star}\left(\mathcal{O}_{Z}\right)\right)
$$

is locally free of $\operatorname{rank} a \cdot n \cdot r^{a}$. Let $B_{\varrho}, \mathfrak{S}^{\prime}$ be the kernel of the tautological quotient

$$
H_{\varrho, \mathfrak{I} \mid \mathfrak{S}^{\prime}} \longrightarrow K_{\mathfrak{I} \mid \mathfrak{S}^{\prime}}
$$

on $\mathfrak{G r}^{q}\left(H_{\varrho, \mathfrak{Q}}\right)$. It is a locally free sheaf of rank $s$ on $\mathfrak{S}^{\prime}$. In fact, it is the tautological subsheaf of $H_{\varrho}, \mathfrak{T} \mid \mathfrak{S}^{\prime}$ when we interpret the Graßmann bundle $\mathfrak{G r}^{q}\left(H_{\varrho}, \mathfrak{Q}\right)$ of $q$-dimensional quotient vector spaces of the fibers of $H_{\varrho, \mathfrak{Q}}$ as the Graßmann bundle $\mathfrak{G r}_{s}\left(H_{\varrho, \mathfrak{Q}}\right)$ of $s$ dimensional sub vector spaces of the fibers of $H_{\varrho, \mathfrak{Q}}$. Finally, we let $\mathfrak{S}$ be the closed subscheme of $\mathfrak{S}^{\prime}$ where the homomorphism

$$
B_{\varrho, \mathfrak{S}^{\prime}} \longrightarrow Q_{\varrho, \mathfrak{I} \mid \mathfrak{S}^{\prime}}
$$

between locally free sheaves is zero. The universal quotients

$$
\chi_{\mathfrak{S}}: V^{\otimes a} \otimes \mathcal{O}_{\mathfrak{S}} \longrightarrow K_{\mathfrak{S}}
$$

and

$$
\kappa \mathfrak{S}: V \otimes \pi_{X}^{\star}\left(\mathcal{O}_{X}(-n)\right) \longrightarrow E_{\mathfrak{S}}
$$

are constructed, by restricting $\chi_{\mathfrak{T}}$ and $\kappa_{\mathfrak{I}}$ to $\mathfrak{S}$ and $\mathfrak{S} \times X$, respectively. Using these families, we may interpret $\mathfrak{\subseteq}$ as a parameter scheme for pairs $\left(\kappa: V \otimes \mathcal{O}_{X}(-n) \longrightarrow E, \Gamma\right)$ in which $(E, \Gamma)$ is a coherent $\varrho$-system of type $\left(\mathcal{O}_{X}, d, s\right)$ and $\kappa$ is a quotient, such that $H^{0}(\kappa(n)): V \longrightarrow H^{0}(E(n))$ is an isomorphism.

Remark 3.2.2. Let $S$ be a scheme of finite type over $\mathbb{C}$ and $\left(E_{S}, \varphi_{S}\right)$ a family of $\delta$-semistable coherent $\varrho$-systems of type $\left(\mathcal{O}_{X}, d, s\right)$ parameterized by $S$. We have

$$
\tilde{\varphi}_{S}: \mathcal{O}_{S \times X}^{\oplus s} \longrightarrow E_{S}^{\otimes a} \longrightarrow\left(E_{S} \otimes \pi_{X}^{\star}\left(\mathcal{O}_{X}(n)\right)\right)^{\otimes a}
$$

By the definition of universally injective, the image of

$$
\pi_{S \star}\left(\widetilde{\varphi}_{S}\right): \mathcal{O}_{S}^{\oplus S} \longrightarrow \pi_{S \star}\left(\left(E_{S} \otimes \pi_{X}^{\star}\left(\mathcal{O}_{X}(n)\right)\right)^{\otimes a}\right)
$$

is a subbundle, and we get the quotient

$$
\pi_{S \star}\left(\left(E_{S} \otimes \pi_{X}^{\star}\left(\mathcal{O}_{X}(n)\right)\right)^{\otimes a}\right) \longrightarrow K_{S}
$$

Now, we may cover $S$ by a family $\left(S_{i}\right)_{i \in I}$ of open subschemes, such that, for each $i \in I$, we have a quotient

$$
\kappa_{S_{i}}: V \otimes \pi_{X}^{\star}\left(\mathcal{O}_{X}(-n)\right) \longrightarrow E_{S \mid S_{i} \times X}
$$


for which

$$
\pi_{S \star}\left(\kappa_{S_{i}} \otimes \pi_{X}^{\star}\left(\mathcal{O}_{X}(n)\right)\right): V \otimes \mathcal{O}_{S} \longrightarrow \pi_{S \star}\left(E_{S \mid S_{i} \times X} \otimes \pi_{X}^{\star}\left(\mathcal{O}_{X}(n)\right)\right)
$$

is an isomorphism. We then obtain the induced quotient

$$
\chi_{S_{i}}: V^{\otimes a} \otimes \mathcal{O}_{S_{i}} \longrightarrow K_{S \mid S_{i}} .
$$

The pair $\left(\kappa_{S_{i}}, \chi_{S_{i}}\right)$ defines a morphism $f_{S_{i}}: S_{i} \longrightarrow \mathfrak{S}$, such that there is an isomorphism between $\kappa_{S_{i}}$ and $\left(f_{S_{i}} \times \operatorname{id}_{X}\right)^{\star}(\kappa \Im)$ which induces an isomorphism between $\chi_{S_{i}}$ and $f_{S}^{\star}\left(\chi \Im_{i}\right)$, $i \in I$.

3.3. The group action and the linearization. The group $\operatorname{GL}(V)$ acts in a natural way on $\mathfrak{S}$, and two points $\left(\kappa_{1}: V \otimes \mathcal{O}_{X}(-n) \longrightarrow E_{1}, \Gamma_{1}\right),\left(\kappa_{2}: V \otimes \mathcal{O}_{X}(-n) \longrightarrow E_{2}, \Gamma_{2}\right) \in \mathfrak{S}$ lie in the same GL $(V)$-orbit if and only if the coherent $\varrho$-systems $\left(E_{1}, \Gamma_{1}\right)$ and $\left(E_{2}, \Gamma_{2}\right)$ are isomorphic (see also Remark 3.3.1, ii). Therefore, constructing the moduli space $\mathscr{C}_{X / \varrho / \mathcal{O}_{X} / d / s}^{\delta}$ for coherent $\varrho$-systems of type $\left(\mathcal{O}_{X}, d, s\right)$ amounts to proving the existence of the categorical quotient for the $\mathrm{GL}(V)$-invariant open subscheme $\mathfrak{U}$ of $\mathfrak{S}$ parameterizing $\delta$-semistable coherent $\varrho$-systems of type $\left(\mathcal{O}_{X}, d, s\right)$. This can be done with the help of geometric invariant theory. For this, we have to find a suitable linearization of the GL( $V)$ action.

Remark 3.3.1. i) We introduce the GL(V)-action, here, because the stack quotient [ $\mathfrak{S} /$ $\mathrm{GL}(V)]$ will be an open part of the moduli stack of coherent $\varrho$-systems, and GL $(V)$ linearized line bundles on $\mathbb{S}$ correspond to line bundles on this part of the moduli stack, just as for the quot scheme (Remark 2.4.1). This will be important for checking when the line bundles in which we will linearize the group action will be ample, using the results from Section 2.4. Since the center $\mathbb{C}^{\star} \cdot \mathrm{id}_{V}$ acts trivially on $\mathfrak{S}$, we will only need the induced $\operatorname{SL}(V)$-action and the $\operatorname{SL}(V)$-linearizations for doing the constructions and computations within geometric invariant theory (see Section 3.4.4).

ii) Let $S$ be a scheme of finite type over $\mathbb{C}$ and $\left(E_{S}^{1}, \varphi_{S}^{1}\right),\left(E_{S}^{2}, \varphi_{S}^{2}\right)$ two families of $\delta$-semistable coherent $\varrho$-systems of type $\left(\mathcal{O}_{X}, d, s\right)$ parameterized by $S$. Suppose that

$$
\kappa_{S}^{i}: V \otimes \pi_{X}^{\star}\left(\mathcal{O}_{X}(-n)\right) \longrightarrow E_{S}^{i}
$$

is a quotient for which

$$
\pi_{S \star}\left(\kappa_{S}^{i} \otimes \pi_{X}^{\star}\left(\mathcal{O}_{X}(n)\right)\right): V \otimes \mathcal{O}_{S} \longrightarrow \pi_{S \star}\left(E_{S}^{i} \otimes \pi_{X}^{\star}\left(\mathcal{O}_{X}(n)\right)\right)
$$

is an isomorphism, $i=1,2$. Let $\left(\psi_{S}, \lambda_{S}\right)$ be an isomorphism between $\left(E_{S}^{1}, \varphi_{S}^{1}\right)$ and $\left(E_{S}^{2}, \varphi_{S}^{2}\right)$ as in Section 1.6 and $K_{S}^{i}$ the quotient bundle introduced before, $i=1,2$. There is an induced isomorphism $\eta_{S}: K_{S}^{1} \longrightarrow K_{S}^{2}$, such that the diagram

$$
\begin{aligned}
\pi_{S \star}\left(\left(E_{S}^{1} \otimes \pi_{X}^{\star}\left(\mathcal{O}_{X}(n)\right)\right)^{\otimes a}\right) & \longrightarrow K_{S}^{1} \\
\pi_{S \star}\left(\left(\psi_{S} \otimes \operatorname{id}_{\pi_{X}^{\star}\left(\mathcal{O}_{X}(n)\right)}\right)^{\otimes a}\right) \downarrow & \downarrow \eta_{S} \\
\pi_{S \star}\left(\left(E_{S}^{2} \otimes \pi_{X}^{\star}\left(\mathcal{O}_{X}(n)\right)\right)^{\otimes a}\right) & \longrightarrow K_{S}^{2}
\end{aligned}
$$

commutes. Next, $\pi_{S \star}\left(\kappa_{S}^{1}\right), \psi_{S} \otimes \mathrm{id}_{\pi_{X}^{\star}\left(\mathcal{O}_{X}(n)\right)}$, and $\pi_{S \star}\left(\kappa_{S}^{2}\right)^{-1}$, yield

$$
\mu_{S}: V \otimes \mathcal{O}_{S} \longrightarrow \pi_{S \star}\left(E_{S}^{1} \otimes \pi_{X}^{\star}\left(\mathcal{O}_{X}(n)\right)\right) \longrightarrow \pi_{S \star}\left(E_{S}^{2} \otimes \pi_{X}^{\star}\left(\mathcal{O}_{X}(n)\right)\right) \longrightarrow V \otimes \mathcal{O}_{S}
$$


The diagrams

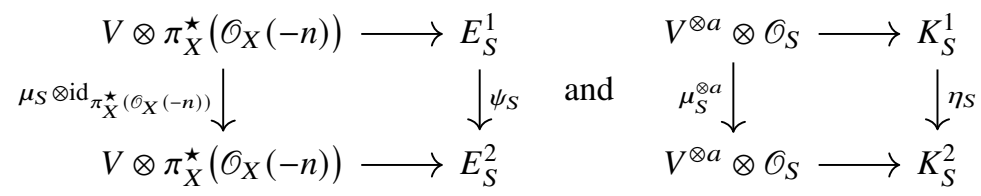

are now commutative. Finally, $\mu_{S}$ defines a morphism $L_{S}: S \longrightarrow \mathrm{GL}(V)$, and, as in Remark 3.2.2, the family $\left(E_{S}^{i}, \varphi_{S}^{i}\right)$ a morphism $f_{S}^{i}: S \longrightarrow \mathbb{S}, i=1,2$. The morphism $f_{S}^{2}$ agrees now with

$$
S \stackrel{\left(L_{S}, f_{S}^{1}\right)}{\longrightarrow} \mathrm{GL}(V) \times \mathfrak{S} \longrightarrow \mathfrak{S} .
$$

3.3.1. The line bundle $\mathscr{L}_{\mathbb{\mathscr { C }}}^{1}$. We denote by $\overline{\mathfrak{Q}}$ the closure of $\mathfrak{Q}$ in the projective quot scheme that parameterizes all quotients $\kappa: V \otimes \mathcal{O}_{X}(-n) \longrightarrow \mathscr{F}$ in which $\mathscr{F}$ is a coherent $\mathscr{O}_{X}$-module of rank $r$ and degree $d$. We have the universal quotient

$$
\kappa_{\bar{Q}}: V \otimes \pi_{X}^{\star}\left(\mathcal{O}_{X}(-n)\right) \longrightarrow \mathscr{F}_{\bar{Q}} .
$$

For $m \in \mathbb{N}$, we set $N_{m}:=H^{0}\left(X, \mathcal{O}_{X}(m)\right)$. Then, we have the homomorphism

$$
\chi_{\overline{\mathfrak{Q}}, m}:\left(V \otimes N_{m}\right) \otimes \mathcal{O}_{\overline{\mathbb{Q}}} \longrightarrow \pi_{\overline{\mathbb{Q}} \star}\left(\mathscr{F}_{\overline{\mathbb{Q}}} \otimes \pi_{X}^{\star}\left(\mathcal{O}_{X}(m+n)\right)\right), \quad m \in \mathbb{N} .
$$

If $m \gg 0$, then the sheaf on the right hand side will be locally free of rank $p_{m}:=$ $r \cdot(m+n+1-g)+d, \chi_{\overline{\mathbb{Q}}, m}$ will be surjective and induce a GL(V)-equivariant embedding of $\overline{\mathfrak{Q}}$ into the Graßmannian $\mathfrak{G}$ of $p_{m}$-dimensional quotients of $V \otimes N_{m}$. Define

$$
\mathcal{O}_{\mathfrak{Q}}(m):=\operatorname{det}\left(\pi_{\overline{\mathbb{Q}} \star}\left(\mathscr{F}_{\bar{Q}} \otimes \pi_{X}^{\star}\left(\mathcal{O}_{X}(m+n)\right)\right)\right), \quad m \gg 0 .
$$

Remark 3.3.2. In (8), we proved the formula

$$
\left(\mathcal{O}_{\overline{\mathfrak{Q}}}(m)^{\otimes p}\right)_{\left.\right|_{\mathfrak{Q}}}=\left(R_{\mathfrak{Q}}^{\otimes p_{0}} \otimes D_{\mathfrak{Q}}^{\otimes(-r)}\right)^{\otimes m}, \quad m \gg 0 .
$$

This shows, in particular, that $R_{\mathfrak{Q}}^{\otimes p_{0}} \otimes D_{\mathfrak{Q}}^{\otimes(-r)}$ is an ample GL $(V)$-linearized line bundle on $\mathfrak{Q}$ which extends to an ample GL $(V)$-linearized line bundle on $\overline{\mathfrak{Q}}$.

We fix a natural number $m$ that is sufficiently large and define $\mathscr{L}_{\mathbb{S}}^{1}$ as the pullback of $\mathcal{O}_{\overline{\mathbb{Q}}}(m)$ to $\mathfrak{S}$.

3.3.2. The line bundle $\mathscr{L}_{\mathbb{S}}^{2}$. The vector bundle $H_{\varrho}, \subseteq$ is linearized with respect to the $\mathrm{GL}(V)$-action, and we define

$$
\mathscr{L}_{\overparen{\Phi}}^{2}:=\operatorname{det}\left(H_{\varrho}, \subseteq\right)^{\vee} .
$$

Remark 3.3.3. i) By construction, $H_{\varrho, \subseteq}$ is the pullback of $H_{\varrho, Q}$ under the morphism $\mathfrak{S} \longrightarrow \mathfrak{Q}$. So, $\mathscr{L}_{\mathbb{S}}^{2}$ is the preimage of $\mathscr{L}_{\mathfrak{Q}}^{2}:=\operatorname{det}\left(H_{\varrho, \mathfrak{Q}}\right)^{\vee}$ under this morphism.

ii) If $a=1$, then, according to Lemma 2.4.5, $\mathscr{L}_{\mathbb{S}}^{2}$ is the trivial line bundle linearized by the character $\operatorname{det}^{-1}$. For the construction of the moduli space with geometric invariant theory, we will only need the SL(V)-action (see Section 3.4.4). This makes $\mathscr{L}_{\mathbb{S}}^{2}$ disappear in the setting of [24]. For $a>1$, we need this line bundle in order to match $h^{0}\left(X, E^{\otimes a}(a \cdot n)\right)$ and $h^{0}(X, E(n))^{a}$ in the formulas that we find (see Sections 3.4.6 to 3.4.8).

3.3.3. The line bundle $\mathscr{L}_{\mathbb{S}}^{3}$. The determinant of $K_{\mathfrak{G}}$ is an ample line bundle on $\mathfrak{G}$ which is linearized with respect to the $\mathrm{GL}(V)$-action. We let $\mathscr{L}_{\mathscr{S}}^{3}$ be the pullback of $\operatorname{det}\left(K_{\mathscr{G}}\right)$ under the morphism $\mathfrak{S} \longrightarrow \mathfrak{G}$. 


\subsubsection{The linearization. Define}

$$
\eta:=\frac{p+a \cdot s \cdot \delta}{m \cdot r \cdot \delta}
$$

and

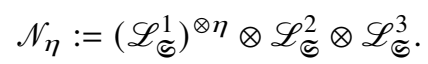

This line bundle carries a natural GL(V)-linearization. We modify this linearization by a suitable rational multiple of the determinant, so that the resulting GL $(V)$-linearized line bundle has weight zero.

3.3.5. The line bundle $\left(\mathscr{L}_{\mathbb{S}}^{1}\right)^{\otimes \eta} \otimes \mathscr{L}_{\mathbb{S}}^{2}$. We use additive notation. Let $w$ be the weight of $\eta \cdot \mathscr{L}_{\overparen{S}}^{1}+\mathscr{L}_{\mathbb{S}}^{2}$. According to (8) and (12), we have - up to a pullback of a line bundle on $\operatorname{Pic}^{d}(X)$ - the identity

$$
\begin{aligned}
& p \cdot\left(\eta \cdot \mathscr{L}_{\mathbb{S}}^{1}+\mathscr{L}_{\overparen{S}}^{2}\right)-w \cdot \mathscr{L}_{0} \\
= & \left(m \cdot \eta-\left(a \cdot(a-1) \cdot r^{a-1}\right) \cdot n-y\right) \cdot\left(p_{0} \cdot R_{\mathfrak{Q}}-r \cdot D_{\mathfrak{Q}}\right) \\
= & \left(\left(\frac{1}{\delta}-\left(a \cdot(a-1) \cdot r^{a-1}\right)\right) \cdot n+\left(\frac{p_{0}+a \cdot s \cdot \delta}{r \cdot \delta}-y\right)\right) \cdot\left(p_{0} \cdot R_{\mathfrak{Q}}-r \cdot D_{\mathfrak{Q}}\right) .
\end{aligned}
$$

Recall from Remark 3.3.2 that $p_{0} \cdot R_{\mathfrak{Q}}-r \cdot D_{\mathfrak{Q}}$ is an ample line bundle. So, in order to grant that $\left(\mathscr{L}_{\overparen{\Phi}}^{1}\right)^{\otimes \eta} \otimes \mathscr{L}_{\overparen{S}}^{2}$ is ample for $n \gg 0$, we need

$$
\delta<\frac{1}{a \cdot(a-1) \cdot r^{a-1}} .
$$

3.4. GIT-Semistability implies semistability. The parameter space that we constructed actually depends on the choice of a natural number $n$. So, we will denote the parameter space constructed with respect to $n \in \mathbb{N}$ by $\mathfrak{S}_{n}$ in this section. The scheme $\mathfrak{S}_{n}$ is endowed with linearized line bundles $\mathscr{L}_{\mathbb{S}_{n}}^{2}$ and $\mathscr{L}_{\mathbb{S}_{n}}^{3}, n \in \mathbb{N}$. In order to define the first linearized line bundle, we also need to fix a natural number $m$. In order to emphasize this, we denote the line bundle defined with respect to $m$ by $\mathscr{L}_{\widetilde{S}_{n}}^{1}(m)$ in the sequel, $m \in \mathbb{N}$. The rational number introduced in (13) also depends on $m$, and will be denoted by $\eta(m), m \in \mathbb{N}$. The linearized line bundle for the GIT construction is, therefore,

$$
\mathcal{N}_{\eta(m)}=\left(\mathscr{L}_{\mathbb{S}_{n}}^{1}(m)\right)^{\otimes \eta} \otimes \mathscr{L}_{\mathbb{S}_{n}}^{2} \otimes \mathscr{L}_{\widetilde{\Phi}_{n}}^{3}, \quad m \gg 0 .
$$

The formula in Section 3.3.5 shows that $\mathcal{N}_{\eta(m)}$ does not depend on the natural number $m$. Therefore, we have a well defined notion of (semi)stability on $\mathfrak{S}_{n}, n \in \mathbb{N}$. The aim of this section is to prove the following result.

Theorem 3.4.1. There are a natural number $n_{1}$, such that, for each natural number $n \geq n_{1}$ and each (semi)stable point $S=\left(\kappa: V \otimes \mathcal{O}_{X}(-n) \longrightarrow E, \Gamma\right) \in \mathfrak{S}_{n}$, the coherent $\varrho$-system $(E, \Gamma)$ is $\delta$-(semi)stable.

Given a projective scheme $\mathfrak{X}$, a reductive linear algebraic group $G$, an action $G \times \mathfrak{X} \longrightarrow$ $\mathfrak{X}$, a linearization $G \times \mathscr{L} \longrightarrow \mathscr{L}$ of this action in the line bundle $\mathscr{L}$ on $\mathfrak{X}$, a point $\mathfrak{x} \in \mathfrak{X}$, and a one parameter subgroup $\lambda: \mathbb{C}^{\star} \longrightarrow G$, we let

$$
\mu_{\mathscr{L}}(\lambda, \mathfrak{z})
$$

be Mumford's numerical function in the convention of [35], (1.9). 
3.4.1. Boundedness of GIT-semistable coherent systems. As $n$ varies, the parameter spaces $\mathfrak{S}_{n}$ form an infinite family of quasi-projective schemes, and this infinite collection will not parameterize a bounded family of vector bundles on $X$. This problem disappears when we restrict to suitable loci of semistable points inside the $\mathfrak{S}_{n}, n \in \mathbb{N}$. More precisely, we have the following property.

Proposition 3.4.2. There is a constant $C_{1}$, such that, for every $n \in \mathbb{N}$, every semistable point $S=\left(\kappa: V \otimes \mathcal{O}_{X}(-n) \longrightarrow E, \Gamma\right) \in \mathfrak{S}_{n}$, and every subbundle $\{0\} \subsetneq F \subset E$, the estimate

is satisfied.

$$
\mu(F) \leq \frac{d}{r}+C_{1}
$$

Proof. Set

$$
\alpha:=\frac{1}{\delta}-a \cdot(a-1) \cdot r^{a-1} \quad \text { and } \quad \beta:=\frac{p_{0}+a \cdot s \cdot \delta}{r \cdot \delta}-y .
$$

Using the formulae for $\mathscr{L}_{\mathbb{S}_{n}}^{1}$ from Remark 3.3.2 and $\eta \cdot \mathscr{L}_{\mathbb{S}_{n}}^{1}+\mathscr{L}_{\mathbb{S}_{n}}^{2}$ from Section 3.3.5, we see that

$$
\frac{m}{p} \cdot\left(\eta \cdot \mathscr{L}_{\mathbb{\Xi}_{n}}^{1}+\mathscr{L}_{\mathbb{E}_{n}}^{2}-w \cdot \mathscr{L}_{0}\right)=(\alpha \cdot n+\beta) \cdot \mathscr{L}_{\mathbb{E}_{n}}^{1}(m)-w^{\prime} \cdot \mathscr{L}_{0} .
$$

Now, let $S=\left(\kappa: V \otimes \mathcal{O}_{X}(-n) \longrightarrow E, \Gamma\right) \in \mathfrak{S}_{n}$ be a semistable point, $F \subset E$ the maximal destabilizing subbundle, and $W_{F} \subset V$ the subspace that maps onto $H^{0}(X, F(n))$ under the isomorphism $H^{0}(X, \kappa(n)): V \longrightarrow H^{0}(X, E(n))$. Pick a basis $\left(v_{1}, \ldots, v_{h_{F}}\right)$ for $H^{0}(X, \kappa(n))^{-1}\left(W_{F}\right)$, complete it to a basis $v=\left(v_{1}, \ldots, v_{p}\right)$ for $V$, and let $\lambda_{F}: \mathbb{C}^{\star} \longrightarrow$ $\mathrm{GL}(V)$ be the one parameter subgroup that is defined with respect to the basis $v$ by the weight vector $g_{p}^{\left(h_{F}\right)}$. We shall make use of the fact that

$$
\mu_{\mathcal{N}_{\eta(m)}}\left(\lambda_{F}, S\right) \geq 0 \text {. }
$$

With the initial observation, this becomes

$$
(\alpha \cdot n+\beta) \cdot \mu_{\mathscr{L}_{\overleftarrow{\Xi}_{n}}^{1}(m)}\left(\lambda_{F}, S\right)+\frac{m}{p} \cdot \mu_{\mathscr{L}_{\mathbb{E}_{n}}^{3}}\left(\lambda_{F}, S\right) \geq 0 .
$$

Let us first find an estimate for $\mu\left(\lambda_{F}, S\right):=\mu_{\mathscr{L}_{\mathbb{E}_{n}}(m)}\left(\lambda_{F}, S\right)$. For this, we denote by $h_{F}(m)$ the dimension of the image of $W_{F} \otimes H^{0}\left(X, \mathcal{O}_{X}(m)\right)$ under the linear map $V \otimes H^{0}\left(X, \mathcal{O}_{X}(m)\right) \longrightarrow H^{0}(X, E(m+n))$ induced by $H^{0}(X, \kappa(n))$ and $H^{0}(X, E(n)) \otimes$ $H^{0}\left(X, \mathcal{O}_{X}(m)\right) \longrightarrow H^{0}(X, E(m+n))$. By (6), we have

$$
\mu\left(\lambda_{F}, S\right)=\left(p-h_{F}\right) \cdot h_{F}(m)-h_{F} \cdot\left(p_{m}-h_{F}(m)\right)=p \cdot h_{F}(m)-h_{F} \cdot p_{m} .
$$

By definition and the Le Potier-Simpson estimate ([28], Lemma 7.1.2) for the semistable vector bundle $F^{9}$

$$
h_{F}(m) \leq h^{0}(X, F(m+n)) \leq \operatorname{deg}(F)+\operatorname{rk}(F) \cdot(m+n+1),
$$

and, obviously,

$$
-h_{F} \leq-\chi(X, F(n))=-\operatorname{deg}(F)+\operatorname{rk}(F) \cdot(n+1-g),
$$

so that

$$
p \cdot(\operatorname{deg}(F)+\operatorname{rk}(F) \cdot(m+n+1))-(\operatorname{deg}(F)+\operatorname{rk}(F) \cdot(n+1-g)) \cdot p_{m} \geq \mu\left(\lambda_{F}, S\right) .
$$

${ }^{9}$ For this, we need $\mu(F)+m+n \geq 0$. We may restrict to the case $\mu(F) \geq d / r$ and, then, for $n \geq$ $-\lfloor\min \{0, d / r\}\rfloor$, this assumption will be met. 
The left hand side is a linear polynomial in $m$ and $n$, and the coefficient of $m$ is

$$
\operatorname{deg}(E) \cdot \operatorname{rk}(F)-\operatorname{deg}(F) \cdot \operatorname{rk}(E) .
$$

The coefficient of $n$ is $r \cdot \operatorname{rk}(F) \cdot g$ and can be bounded from above by $r \cdot(r-1) \cdot g$. The constant term is $d \cdot \operatorname{rk}(F) \cdot g+r \cdot \operatorname{rk}(F) \cdot g \cdot(1-g)$ and can be bounded from above by $d_{+} \cdot(r-1) \cdot g, d_{+}:=\max \{d, 0\}$.

The linearized line bundle $\mathscr{L}_{\mathfrak{S}_{n}}^{3}$ is induced by the Plücker embedding of $\mathfrak{G}$ into

$$
\mathbb{P}\left(\bigwedge^{p(a)-s} V^{\otimes a}\right)
$$

For this reason,

$$
\mu_{\mathscr{L}_{\mathbb{E}_{n}}^{3}}\left(\lambda_{F}, S\right) \leq(p(a)-s) \cdot a \cdot\left(p-h_{F}\right) \leq(p(a)-s) \cdot a \cdot p
$$

and

$$
\frac{m}{p} \cdot \mu_{\mathscr{L}_{\mathbb{E}_{n}}^{3}}\left(\lambda_{F}, S\right) \leq r^{a} \cdot a^{2} \cdot m \cdot n+a \cdot\left(r^{a} \cdot(1-g)+a \cdot r^{a-1} \cdot d-s\right) \cdot m .
$$

Altogether, we see that

$$
\begin{aligned}
& (\alpha \cdot n+\beta) \cdot\left((d \cdot \operatorname{rk}(F)-\operatorname{deg}(F) \cdot r) \cdot m+r \cdot(r-1) \cdot g \cdot n+d_{+} \cdot(r-1) \cdot g\right) \\
& +r^{a} \cdot a^{2} \cdot m \cdot n+a \cdot\left(r^{a} \cdot(1-g)+a \cdot r^{a-1} \cdot d-s\right) \cdot m \\
\geq & \frac{m}{p} \cdot \mu_{\mathcal{N}_{\eta(m)}}\left(\lambda_{F}, S\right) \geq 0 .
\end{aligned}
$$

We claim that, for $n \gg 0$, this implies

$$
\alpha \cdot(d \cdot \operatorname{rk}(F)-\operatorname{deg}(F) \cdot r)+r^{a} \cdot a^{2} \geq 0,
$$

i.e.,

$$
\mu_{\max }(E)=\mu(F) \leq \frac{d}{r}+\frac{r^{a-1} \cdot a^{2}}{\alpha \cdot \operatorname{rk}(F)} \leq \frac{d}{r}+C_{1}, \quad C_{1}:=\frac{r^{a-1} \cdot a^{2}}{\alpha} .
$$

Let us assume that our claim is false. We set

$$
\begin{aligned}
\Delta & :=d \cdot \operatorname{rk}(F)-\operatorname{deg}(F) \cdot r, \\
K_{1} & :=r \cdot(r-1) \cdot g \cdot n+d_{+} \cdot(r-1) \cdot g, \\
K_{2} & :=r^{a} \cdot a^{2}, \\
K_{3} & :=a \cdot\left(r^{a} \cdot(1-g)+a \cdot r^{a-1} \cdot d-s\right) .
\end{aligned}
$$

Note that $K_{1}, K_{2}$, and $K_{3}$ depend only on $a, r, d$, and $s$. We know that

$$
\left(\left(\alpha \cdot \Delta+K_{2}\right) \cdot n+\left(\beta \cdot \Delta+K_{3}\right)\right) \cdot m+K_{1} \geq 0 .
$$

There is a natural number $K_{4}$ which depends only on $a, r, d$, s, and $\delta$, such that

Estimate (15) implies

$$
\alpha \cdot \Delta+K_{2} \leq-\frac{1}{K_{4}}
$$

$$
\left(-\frac{n}{K_{4}}+\beta \cdot \Delta+K_{3}\right) \cdot m+K_{1} \geq 0 .
$$

There is a natural number $n_{1}^{\prime}$, depending only on $a, r, d, s$, and $\delta$, such that

$$
\forall n \geq n_{1}^{\prime}: \quad-\frac{n}{K_{4}}+\beta \cdot \Delta+K_{3} \leq-2 \cdot K_{1} .
$$


Because of (16), we have

$$
m \leq \frac{-K_{1}}{-\frac{n}{K_{4}}+\beta \cdot \Delta+K_{3}} \leq \frac{1}{2} .
$$

This is impossible, because $m$ is a positive rational number.

For the finitely many remaining values of $n$, namely, $1, \ldots, n_{1}^{\prime}-1$, we don't need to worry, because, for these, $E$ lives in a bounded family parameterized by the quasi-projective scheme $\mathfrak{\mathfrak { S }}_{1} \sqcup \cdots \sqcup \mathfrak{\mathfrak { S }}_{n_{1}^{\prime}-1}$. This finishes the arguments.

3.4.2. A finiteness result. Given a vector bundle $E$ and a weighted filtration $\left(E_{\bullet}, \alpha_{\bullet}\right)$ of $E, E_{\bullet}:\{0\} \subsetneq E_{1} \subsetneq \cdots \subsetneq E_{l} \subsetneq E$, the tuple $t\left(E_{\bullet}, \alpha_{\bullet}\right):=\left(r_{\bullet}, \alpha_{\bullet}\right)$ with $r_{\bullet}:=\left(r_{1}, \ldots, r_{l}\right)$ and $r_{i}:=\operatorname{rk}\left(E_{i}\right), i=1, \ldots, l$, is the type of $\left(E_{\bullet}, \alpha_{\bullet}\right)$. By means of a straightforward generalization of the definitions in Example 2.2.1, ii), iii), we define the relation " $\leq$ " on weighted filtrations of $E_{\bullet}$ and “+”. Now, let $(E, \Gamma)$ be a coherent $\varrho$-system and $\left(E_{\bullet}^{\prime}, \alpha_{\bullet}^{\prime}\right)$, $\left(E_{\bullet}^{\prime \prime}, \alpha_{\bullet}^{\prime \prime}\right)$, and $\left(E_{\bullet}, \alpha_{\bullet}\right)$ weighted filtrations of $E$, such that

$$
\left(E_{\bullet}, \alpha_{\bullet}\right)=\left(E_{\bullet}^{\prime}, \alpha_{\bullet}^{\prime}\right)+\left(E_{\bullet}^{\prime \prime}, \alpha_{\bullet}^{\prime \prime}\right) .
$$

Let $\left(B_{\bullet}^{\prime}, \beta_{\bullet}^{\prime}\right),\left(B_{\bullet}^{\prime \prime}, \beta_{\bullet}^{\prime \prime}\right)$, and $\left(B_{\bullet}, \beta_{\bullet}\right)$, respectively, be the induced weighted filtrations of $E^{\otimes a}$. If also

then, clearly,

$$
\left(B_{\bullet}, \beta_{\bullet}\right)=\left(B_{\bullet}^{\prime}, \beta_{\bullet}^{\prime}\right)+\left(B_{\bullet}^{\prime \prime}, \beta_{\bullet}^{\prime \prime}\right),
$$

$$
\begin{aligned}
& M\left(E_{\bullet}, \alpha_{\bullet}\right)+\delta \cdot \mu_{(E, \Gamma)}\left(E_{\bullet}, \alpha_{\bullet}\right)= \\
& M\left(E_{\bullet}^{\prime}, \alpha_{\bullet}^{\prime}\right)+\delta \cdot \mu_{(E, \Gamma)}\left(E_{\bullet}^{\prime}, \alpha_{\bullet}^{\prime}\right)+M\left(E_{\bullet}^{\prime \prime}, \alpha_{\bullet}^{\prime \prime}\right)+\delta \cdot \mu_{(E, \Gamma)}\left(E_{\bullet}^{\prime \prime}, \alpha_{\bullet}^{\prime \prime}\right) .
\end{aligned}
$$

We would like to use this additivity property to simplify the notion of $\delta$-semistability in a way that is useful for theoretical considerations and probably also for concrete computations with the semistability concept as in [35], Section 2.3.3.

Proposition 3.4.3. Let $(E, \Gamma)$ be a coherent $\varrho$-system and $\delta \in \mathbb{Q}_{>0}$. Then, $(E, \Gamma)$ is $\delta$-(semi)stable if and only if it satisfies the condition of $\delta$-(semi)stability for all weighted filtrations $\left(E_{\bullet}, \alpha_{\bullet}\right)$ with $^{10}$

$$
t\left(E_{\bullet}, \alpha_{\bullet}\right) \in \mathscr{T}(\varrho) .
$$

Here, finiteness refers to the finiteness of the number of weight vectors $\alpha_{\bullet}$ that are needed for testing semistability.

Proof. Let $e=\left(e_{1}, \ldots, e_{r}\right)$ be the standard basis of $\mathbb{C}^{r}, S \subset \mathrm{SL}_{r}(\mathbb{C})$ the subgroup of diagonal matrices, and $S \subset A \subset \mathrm{SL}_{r}(\mathbb{C})$ the Borel subgroup that corresponds, in the notation of Example 2.2.1, to the identity. The $a$-fold tensor power $\left(\mathbb{C}^{r}\right)^{\otimes a}$ has the basis $\left(e_{\underline{i}}:=e_{i_{1}} \otimes \cdots \otimes e_{i_{a}}, \underline{i}=\left(i_{1}, \ldots, i_{a}\right) \in\{1, \ldots, r\}^{\times a}\right)$, ordered, e.g., by the lexicographic ordering. We use this ordered basis to identify $\left(\mathbb{C}^{r}\right)^{\otimes a}$ with $\mathbb{C}^{N}, N:=r^{a}$, let $\varrho: \mathrm{GL}_{r}(\mathbb{C}) \longrightarrow \mathrm{GL}_{N}(\mathbb{C})$ be the $a$-fold tensor power of the standard representation, and $\eta: \mathrm{SL}_{r}(\mathbb{C}) \longrightarrow \mathrm{SL}_{N}(\mathbb{C})$ the induced representation. Under these circumstances, $\eta(S)$ is contained in the subgroup $T \subset \mathrm{SL}_{N}(\mathbb{C})$ of diagonal matrices.

Now, let $\left(E_{\bullet}, \alpha_{\bullet}\right)$ be a weighted filtration of $E, E_{\bullet}:\{0\} \subsetneq E_{1} \subsetneq \cdots \subsetneq E_{l} \subsetneq E$, $\alpha_{\bullet}=\left(\alpha_{1}, \ldots, \alpha_{l}\right)$. Since, for $\ell \in \mathbb{Z}$, the semistability condition evaluated at $\left(E_{\bullet}, \alpha_{\bullet}\right)$ is equivalent to the semistability condition evaluated at $\left(E_{\bullet}, \ell \cdot \alpha_{\bullet}\right)$, we may assume without loss of generality that $\alpha_{i} \in(1 / r) \cdot \mathbb{Z}$. Let $r_{i}:=\operatorname{rk}\left(E_{i}\right), i=1, \ldots, l$. Then, $\sum_{i=1}^{l} \alpha_{i} \cdot g_{r}^{\left(r_{i}\right)}$ is an integral weight vector, and we define $\lambda$ as the one parameter subgroup of $\operatorname{SL}_{r}(\mathbb{C})$ defined

${ }^{10}$ Recall Definition (5) 
by this weight vector and the standard basis $e$. The $\mathbb{Q}_{>0}$-weighted flag $\left(W_{\bullet}, \alpha_{\bullet}\right)$ associated with $\lambda$ features

$$
W_{\bullet}: \quad\{0\} \subseteq\left\langle e_{1}, \ldots, e_{r_{1}}\right\rangle \subsetneq \cdots \subsetneq\left\langle e_{1}, \ldots, e_{r_{l}}\right\rangle \subsetneq \mathbb{C}^{r}
$$

and $A \subset Q_{\mathrm{SL}_{r}(\mathbb{C})}(\lambda)$, i.e., $\lambda \in \overline{\mathscr{W}}(A, S)$. As described in Section 2.2, there is an index $k \in\{1, \ldots, K\}$ with $\lambda \in \overline{\mathscr{V}}_{k}$. Suppose that $\lambda^{\prime}, \lambda^{\prime \prime} \in \overline{\mathscr{V}}_{k} \cap X_{\star}(S)$ are one parameter subgroups with $\lambda^{\prime}+\lambda^{\prime \prime}=\lambda$. As noted in Example 2.2.1, iii), the $\mathbb{Q}_{>0}$-weighted flags $\left(W_{\bullet}^{\prime}, \alpha_{\bullet}^{\prime}\right)$ and $\left(W_{\bullet}^{\prime \prime}, \alpha_{\bullet}^{\prime \prime}\right)$ associated with $\lambda^{\prime}$ and $\lambda^{\prime \prime}$, respectively, satisfy $\left(W_{\bullet}^{\prime}, \alpha_{\bullet}^{\prime}\right) \leq\left(W_{\bullet}, \alpha_{\bullet}\right)$, $\left(W_{\bullet}^{\prime \prime}, \alpha_{\bullet}^{\prime \prime}\right) \leq\left(W_{\bullet}, \alpha_{\bullet}\right)$, and $\left(W_{\bullet}^{\prime}, \alpha_{\bullet}^{\prime}\right)+\left(W_{\bullet}^{\prime \prime}, \alpha_{\bullet}^{\prime \prime}\right)=\left(W_{\bullet}, \alpha_{\bullet}\right)$. We define $Q_{\mathrm{GL}_{r}(\mathbb{C})}\left(\lambda^{\prime}\right)$, $Q_{\mathrm{GL}_{r}(\mathbb{C})}\left(\lambda^{\prime \prime}\right)$, and $Q_{\mathrm{GL}_{r}(\mathbb{C})}(\lambda)$ by Formula (3). These are the $\mathrm{GL}_{r}(\mathbb{C})$-stabilizers of the flags $W_{\bullet}^{\prime}, W_{\bullet}^{\prime \prime}$, and $W_{\bullet}$, respectively. We point out that

$$
Q_{\mathrm{GL}_{r}(\mathbb{C})}(\lambda) \subset Q_{\mathrm{GL}_{r}(\mathbb{C})}\left(\lambda^{\prime}\right) \cap Q_{\mathrm{GL}_{r}(\mathbb{C})}\left(\lambda^{\prime \prime}\right) .
$$

Let $\mathscr{P}(E)$ be the frame bundle of $E$. The filtration $E \bullet$ of $E$ corresponds to a section

$$
\sigma: X \longrightarrow \mathscr{P}(E) / Q_{\mathrm{GL}_{r}(\mathbb{C})}(\lambda)
$$

The induced sections

$$
\begin{array}{r}
\sigma^{\prime}: X \stackrel{\sigma}{\longrightarrow} \mathscr{P}(E) / Q_{\mathrm{GL}_{r}(\mathbb{C})}(\lambda) \longrightarrow \mathscr{P}(E) / Q_{\mathrm{GL}_{r}(\mathbb{C})}\left(\lambda^{\prime}\right), \\
\sigma^{\prime \prime}: X \stackrel{\sigma}{\longrightarrow} \mathscr{P}(E) / Q_{\mathrm{GL}_{r}(\mathbb{C})}(\lambda) \longrightarrow \mathscr{P}(E) / Q_{\mathrm{GL}_{r}(\mathbb{C})}\left(\lambda^{\prime \prime}\right)
\end{array}
$$

yield filtrations $E_{\bullet}^{\prime}$ and $E_{\bullet}^{\prime \prime}$, respectively. Note that the length $l^{\prime}$ of $E_{\bullet}^{\prime}$ matches the length of $W_{\bullet}^{\prime}$ and the rank of the $i$-th entry of $E_{\bullet}^{\prime}$ the dimension of the $i$-th entry of $W_{\bullet}^{\prime}, i=1, \ldots, l^{\prime}$. The analogous statement holds for $E_{\bullet}^{\prime \prime}$ and $W_{\bullet}^{\prime \prime}$. So, we get the weighted filtrations $\left(E_{\bullet}^{\prime}, \alpha_{\bullet}^{\prime}\right)$ and $\left(E_{\bullet}^{\prime \prime}, \alpha_{\bullet}^{\prime \prime}\right)$ of $E$, and it is apparent from the construction that

$$
\left(E_{\bullet}^{\prime}, \alpha_{\bullet}^{\prime}\right)+\left(E_{\bullet}^{\prime \prime}, \alpha_{\bullet}^{\prime \prime}\right)=\left(E_{\bullet}, \alpha_{\bullet}\right) .
$$

By construction, there is a Borel subgroup $T \subset B \subset \mathrm{SL}_{N}(\mathbb{C})$ with $\eta_{\star}\left(\overline{\mathscr{V}}_{k}\right) \subset \overline{\mathscr{W}}(B, T)$. The one parameter subgroup $\eta_{\star}(\lambda)$ defines a $\mathbb{Q}_{>0}$-weighted flag $\left(V_{\bullet}, \beta_{\bullet}\right)$ in $\mathbb{C}^{N}$. For the frame bundles $\mathscr{P}(E)$ and $\mathscr{P}\left(E^{\otimes a}\right)$, we have an inclusion $\mathscr{P}(E) \hookrightarrow \mathscr{P}\left(E^{\otimes a}\right)$, and

$$
\varrho_{\star}(\sigma): X \stackrel{\sigma}{\longrightarrow} \mathscr{P}(E) / Q_{\mathrm{GL}_{r}(\mathbb{C})}(\lambda) \longrightarrow \mathscr{P}\left(E^{\otimes a}\right) / Q_{\mathrm{GL}_{N}(\mathbb{C})}(\varrho \circ \lambda)
$$

defines a filtration $B_{\bullet}$ of $E^{\otimes a}$. The length $m$ of $B_{\bullet}$ matches the length of $V_{\bullet}$ and the rank of the $i$-th entry of $B_{\bullet}$ the dimension of the $i$-th entry of $V_{\bullet}, i=1, \ldots, m$. So, we get the weighted filtration $\left(B_{\bullet}, \beta_{\bullet}\right)$. This is the weighted filtration of $E^{\otimes a}$ associated with $\left(E_{\bullet}, \alpha_{\bullet}\right)$. Likewise, $\left(E_{\bullet}^{\prime}, \alpha_{\bullet}^{\prime}\right)$ and $\left(E_{\bullet}^{\prime \prime}, \alpha_{\bullet}^{\prime \prime}\right)$ lead to weighted filtrations $\left(B_{\bullet}^{\prime}, \beta_{\bullet}^{\prime}\right)$ and $\left(B_{\bullet}^{\prime \prime}, \beta_{\bullet}^{\prime \prime}\right)$ of $E^{\otimes a}$. Since we have the commutative diagram

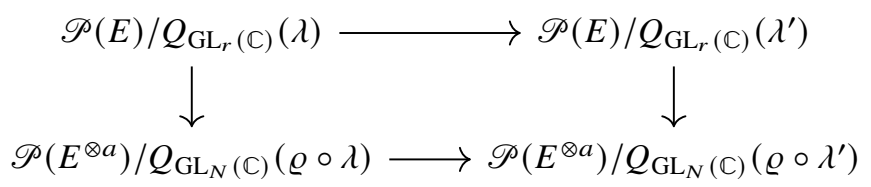

and a similar diagram for $\lambda^{\prime \prime}$, the same discussion as before shows

$$
\left(B_{\bullet}, \beta_{\bullet}\right)=\left(B_{\bullet}^{\prime}, \beta_{\bullet}^{\prime}\right)+\left(B_{\bullet}^{\prime \prime}, \beta_{\bullet}^{\prime \prime}\right) \text {. }
$$

Now, let $\lambda_{1}^{k}, \ldots, \lambda_{L_{k}}^{k}$ be the minimal integral generators of the edges of the cone $\overline{\mathscr{V}}_{k}$. Then, there are non-negative rational numbers $a_{1}, \ldots, a_{L_{k}}$, such that

$$
\lambda=a_{1} \cdot \lambda_{1}^{k}+\cdots+a_{L_{k}} \cdot \lambda_{L_{k}}^{k}
$$


Again, we may multiply $\lambda$ by a positive integer and assume that $a_{1}, \ldots, a_{L_{k}}$ are natural numbers. So, we get weighted filtrations $\left(B_{\bullet}^{1}, \alpha_{\bullet}^{1}\right), \ldots,\left(B_{\bullet}^{L_{k}}, \alpha_{\bullet}^{L_{k}}\right)$ of $E^{\otimes a}$ with

$$
\left(B_{\bullet}, \beta_{\bullet}\right)=\sum_{i=1}^{L_{k}} a_{i} \cdot\left(B_{\bullet}^{i}, \beta_{\bullet}^{i}\right)
$$

Since $t\left(B_{\bullet}^{i}, \beta_{\bullet}^{i}\right)=t\left(\lambda_{i}^{k}\right) \in \mathscr{T}(\varrho), i=1, \ldots, L_{k}$, this and (17) yield the claim.

3.4.3. Bounded filtrations. Given a constant $C$, the family $\mathfrak{B}_{0}(C)$ of isomorphy classes $[F]$ of vector bundles $F$ of rank $r$ and degree $d$ with $\mu_{\max }(F) \leq(d / r)+C$ is bounded. Given a second constant $D$, the family $\mathfrak{B}_{1}(C, D)$ of vector bundles $[G]$, such that $\mu(G) \geq d / r-D$ and $G$ is a subbundle of a vector bundle $F$ with $[F] \in \mathfrak{B}_{0}(C)$ is bounded, as well. Let $C_{1}$ be the (positive) constant from Proposition 3.4.2.

Proposition 3.4.4. There is a constant $C_{2}$, such that a coherent $\varrho$-system $(E, \Gamma)$ of type $\left(\mathcal{O}_{X}, d, s\right)$ with $[E] \in \mathfrak{B}_{0}\left(C_{1}\right)$ is $\delta$-(semi)stable if and only if it satisfies the condition of $\delta$-(semi)stability for weighted filtrations $\left(E_{\bullet}, \alpha_{\bullet}\right)$ of $E$ with $\left[E_{i}\right] \in \mathfrak{B}_{1}\left(C_{1}, C_{2}\right), i=1, \ldots, l$, $E_{\bullet}:\{0\} \subsetneq E_{1} \subsetneq \cdots \subsetneq E_{l} \subsetneq E$.

Proof. By Proposition 3.4.3, we need to check the condition of $\delta$-(semi)stability only for weighted filtrations $\left(E_{\bullet}, \alpha_{\bullet}\right)$ of $E$ with $t\left(E_{\bullet}, \alpha_{\bullet}\right) \in \mathscr{T}(\varrho)$. So, we pick a type $t=\left(r_{\bullet}, \alpha_{\bullet}\right)=$ $\left(\left(r_{1}, \ldots, r_{l(t)}\right),\left(\alpha_{1}, \ldots, \alpha_{l(t)}\right)\right) \in \mathscr{T}(\varrho)$, an index $i_{0} \in\{1, \ldots, l(t)\}$, and let $\left(E_{\bullet}, \alpha_{\bullet}\right)$ be a weighted filtration of $E$ of type $t$ with $\left[E_{i_{0}}\right] \notin \mathfrak{B}_{1}\left(C_{2}\right)$.

First,

$$
\begin{aligned}
& \sum_{\substack{i \in\{, l(t)\}: \\
i \neq i_{0}}} \alpha_{i} \cdot\left(d \cdot \operatorname{rk}\left(E_{i}\right)-\operatorname{deg}\left(E_{i}\right) \cdot r\right) \\
& \geq \sum_{\substack{i \in\{1, \ldots, l(t)\}: \\
i \neq i_{0}}} \alpha_{i} \cdot\left(d \cdot \operatorname{rk}\left(E_{i}\right)-\left(\frac{d}{r}+C_{1}\right) \cdot \operatorname{rk}\left(E_{i}\right) \cdot r\right) \\
& =-C_{1} \cdot \sum_{\substack{i \in\{1, \ldots, l(t)\}: \\
i \neq i_{0}}} \alpha_{i} \cdot \operatorname{rk}\left(E_{i}\right) \cdot r .
\end{aligned}
$$

Knowing $t$, we may easily define a positive constant $K_{1}^{\prime}\left(r, d, C_{1}, t, i_{0}\right)$, such that the latter term can be bounded from below by $-K_{1}^{\prime}\left(r, d, C_{1}, t, i_{0}\right)$. Since there are only finitely choices for $t$ and $i_{0}$, we can find a positive constant $K_{1}\left(r, d, C_{1}\right)$ which depends only on $r$, and $C_{1}$ with $K_{1}^{\prime}\left(r, d, C_{1}, t, i_{0}\right) \leq K_{1}\left(r, d, C_{1}\right), t \in \mathscr{T}(\varrho), i_{0}=1, \ldots, l(t)$.

Next, let $\left(B_{\bullet}, \beta_{\bullet}\right)$ be the induced weighted filtration of $E^{\otimes a}$ and $m$ the length of $B_{\bullet}$. Recall that we use $b_{j}:=\operatorname{dim}_{\mathbb{C}}\left(H^{0}\left(X, B_{j}\right) \cap \Gamma\right), j=1, \ldots, m$. Clearly, $b_{j} \leq s, j=1, \ldots, m$. So, we have

$$
\begin{aligned}
\mu_{(E, \Gamma)}\left(E_{\bullet}, \alpha_{\bullet}\right) & =\sum_{j=1}^{m} \beta_{j} \cdot\left(\operatorname{rk}\left(B_{j}\right) \cdot s-b_{j} \cdot a \cdot r\right) \\
& \geq-s \cdot \sum_{j=1}^{m} \beta_{j} \cdot a \cdot r=-s \cdot a \cdot\left(\gamma_{r}-\gamma_{1}\right)=-s \cdot a \cdot \sum_{i=1}^{l(t)} \alpha_{i} \cdot r .
\end{aligned}
$$


Here, $\sum_{i=1}^{l} \alpha_{i} \cdot \gamma_{r}^{\left(\mathrm{rk}\left(E_{i}\right)\right)}=\left(\gamma_{1}, \ldots, \gamma_{r}\right)$, and, so, $\sum_{j=1}^{m} \beta_{j} \cdot \gamma_{a \cdot r}^{\left(\mathrm{rk}\left(B_{j}\right)\right)}=\left(a \cdot \gamma_{1}, \ldots, a \cdot \gamma_{r}\right) .{ }^{11}$ There is positive constant $K_{2}^{\prime}(s, t)$, such that the last expression is bounded from below by $-K_{2}^{\prime}(s, t)$. Again, there is a constant $K_{2}(r, a, s)$, depending only $\varrho$, i.e., on $r$ and $a$, as well as on $s$, with $K_{2}^{\prime}(s, t) \leq K_{2}(r, a, s), t \in \mathscr{T}(\varrho)$.

Finally,

$$
\begin{aligned}
& \alpha_{i_{0}} \cdot\left(d \cdot \operatorname{rk}\left(E_{i_{0}}\right)-\operatorname{deg}\left(E_{i_{0}}\right) \cdot r\right) \\
\geq & \alpha_{i_{0}} \cdot\left(d \cdot \operatorname{rk}\left(E_{i_{0}}\right)-\left(\frac{d}{r}-C_{2}\right) \cdot \operatorname{rk}\left(E_{i_{0}}\right) \cdot r\right) \\
= & \alpha_{i_{0}} \cdot \operatorname{rk}\left(E_{i_{0}}\right) \cdot C_{2} \\
\geq & \frac{C_{2}}{r} .
\end{aligned}
$$

Here, we have used that $\alpha_{i_{0}} \in(1 / r) \cdot \mathbb{Z}$. Altogether, we see that

$$
M\left(E_{\bullet}, \alpha_{\bullet}\right)+\delta \cdot \mu_{(E, \Gamma)}\left(E_{\bullet}, \alpha_{\bullet}\right) \geq-K_{1}\left(r, d, C_{1}\right)-K_{2}(r, a, s)+\frac{\delta}{r} \cdot C_{2} .
$$

Since the set $\mathscr{T}(\varrho)$ depends only on $r$ and $a$ and the constants $K_{1}, K_{2}$, and $K_{3}$ depend only on $a, r, d, s$, and $C_{1}$ which, in turn, depends only on $a, r$, and $\delta$, we see that we can find a constant $C_{2}$, depending only on $a, r, d, s$, and $\delta$, such that the expression $-K_{1}\left(r, d, C_{1}\right)-K_{2}(r, a, s)+(\delta / r) \cdot C_{2}$ is positive.

3.4.4. Proof of Theorem 3.4.1. To ease notation, we will drop the indices $n$ and $m$ from the symbols for the parameter space and its linearized line bundles again. The center $\mathbb{C}^{\star} \cdot \operatorname{id}_{V}$ of $\operatorname{GL}(V)$ acts trivially on $\mathfrak{\subseteq}$ and the line bundle $\mathcal{N}_{\eta}$. For this reason, we look from now on at the induced action of the group $\operatorname{SL}(V)$. The subspace $\Gamma \subset H^{0}\left(X, E^{\otimes a}\right)$ defines $(p(a)-s)$-dimensional quotients $\widehat{\chi}: H^{0}\left(X, E(n)^{\otimes a}\right) \longrightarrow K$ and $\chi: V^{\otimes a} \longrightarrow K$. We assume that the bounded family $\mathfrak{B}$ defined at the beginning of Section 3.2 contains the family $\mathfrak{B}_{1}\left(C_{1}, C_{2}\right)$ from Proposition 3.4.4. According to that proposition, the condition of $\delta$-(semi)stability for $(E, \Gamma)$ has to be checked only for weighted filtrations of $E$, such that the isomorphy classes of the subbundles of $E$ occuring in the filtration belong to $\mathfrak{B}$.

We pick a weighted filtration $\left(E_{\bullet}, \alpha_{\bullet}\right)$ of $E$, such that $E_{i} \in \mathfrak{B}, i=1, \ldots, l$, and a one parameter subgroup $\lambda: \mathbb{C}^{\star} \longrightarrow \mathrm{GL}(V)$, such that its $\mathbb{Q}_{>0}$-weighted flag is $\left(V_{\bullet}, \alpha \cdot \alpha_{\bullet}\right)$ with

$$
H^{0}(X, \kappa(n))\left(V_{i}\right)=H^{0}\left(X, E_{i}(n)\right), \quad i=1, \ldots, l .
$$

We denote by $\left(E_{\bullet}, \gamma_{\bullet}\right)$ and $\left(V_{\bullet}, \widetilde{\gamma}_{\bullet}\right)$ the corresponding $\mathbb{Z}$-weighted filtration and flag, respectively. Let $\left(H_{\bullet}, \widetilde{\varepsilon}_{\bullet}\right)$ and $\left(H_{\bullet}, \widetilde{\beta}_{\bullet}\right)$ be the $\mathbb{Z}$-weighted and $\mathbb{Q}_{>0}$-weighted flag, respectively, associated with the one parameter subgroup given as the composition of $\lambda$ and the homomorphism GL $(V) \longrightarrow \mathrm{GL}\left(V^{\otimes a}\right)$.

Let us start with a tuple $\underline{i}=\left(i_{1}, \ldots, i_{a}\right) \in\{1, \ldots, l+1\}^{\times a}$ and set

$$
V_{\underline{i}}:=V_{i_{1}} \otimes \cdots \otimes V_{i_{a}} \quad \text { and } \quad E_{\underline{i}}:=E_{i_{1}} \otimes \cdots \otimes E_{i_{a}} .
$$

Remark 3.4.5. Our assumptions imply that the quotient $V^{\otimes a} \longrightarrow H^{0}\left(X, E(n)^{\otimes a}\right)$ maps $V_{\underline{i}}$ surjectively onto $H^{0}\left(X, E_{\underline{i}}(a \cdot n)\right)$.

${ }^{11}$ This just indicates that the first weight is $a \cdot \gamma_{1}$ and the last one $a \cdot \gamma_{r}$. Apparently, not all the intermediate weights will be multiples of some $\gamma_{i}, i=2, \ldots, r-1$. 
The space $V_{\underline{i}}$ is a subspace of the space $H(\underline{i})$ in the flag $H_{\bullet}$ that is associated with the weight

$$
\widetilde{\varepsilon}_{\underline{i}}:=\widetilde{\gamma}_{i_{1}}+\cdots+\widetilde{\gamma}_{i_{a}}=a \cdot \widetilde{\gamma}_{1}+\sum_{k=1}^{a}\left(\widetilde{\gamma}_{i_{k}}-\widetilde{\gamma}_{1}\right)=a \cdot \widetilde{\gamma}_{1}+\sum_{k=1}^{a}\left(\sum_{s=1}^{i_{k}-1} \alpha_{s} \cdot p\right) .
$$

Define

$$
I(\underline{i}):=\left\{\underline{\iota}=\left(\iota_{1}, \ldots, \iota_{a}\right) \in\{1, \ldots, l+1\}^{\times a} \mid \widetilde{\gamma}_{\iota_{1}}+\cdots+\widetilde{\gamma}_{\iota_{a}} \leq \widetilde{\varepsilon}_{\underline{i}}\right\} .
$$

Then,

$$
H(\underline{i})=\sum_{\underline{\underline{\iota}} \in I(\underline{i})} V_{\underline{\iota}},
$$

the sum being taken inside $V^{\otimes a}$. Likewise, $E_{\underline{i}}$ is contained in the bundle $B(\underline{i})$ in the filtration $B$. that corresponds to the weight

$$
\varepsilon_{\underline{i}}=a \cdot \gamma_{1}+\sum_{k=1}^{a}\left(\sum_{s=1}^{i_{k}-1} \alpha_{s} \cdot r\right)
$$

Setting

$$
J(\underline{i}):=\left\{\underline{\iota}=\left(\iota_{1}, \ldots, \iota_{a}\right) \in\{1, \ldots, l+1\}^{\times a} \mid \gamma_{\iota_{1}}+\cdots+\gamma_{\iota_{a}} \leq \varepsilon_{\underline{i}}\right\},
$$

we get

$$
B(\underline{i})=\sum_{\underline{\underline{\iota}} \in J(\underline{i})} B_{\underline{\underline{\iota}}},
$$

the sum being taken inside $E^{\otimes a}$. It is apparent from (18) and (19) that

$$
I(\underline{i})=J(\underline{i}) \text {. }
$$

Remark 3.4.6. The assumptions at the beginning of this part show that the quotient $V^{\otimes a} \longrightarrow$ $H^{0}\left(X, E(n)^{\otimes a}\right)$ maps $H(\underline{i})$ surjectively onto $H^{0}(X, B(\underline{i})(a \cdot n))$.

Write $\widetilde{\varepsilon}_{\bullet}=\left(\widetilde{\varepsilon}_{1}, \ldots, \widetilde{\varepsilon}_{\widetilde{\mu}+1}\right)$ and $\varepsilon_{\bullet}=\left(\varepsilon_{1}, \ldots, \varepsilon_{\mu+1}\right)$. From the above observations, we infer $\widetilde{\mu}=\mu$, and, given $j \in\{1, \ldots, \mu+1\}$ and $\underline{i}$ with $\widetilde{\varepsilon}_{j}=\widetilde{\varepsilon}_{\underline{i}}$, we have $\varepsilon_{j}=\varepsilon_{\underline{i}}$. Involving (18) and (19), we draw the important conclusion that

$$
\widetilde{\beta}_{j} \cdot p^{a} \cdot r=\left(\widetilde{\varepsilon}_{j+1}-\widetilde{\varepsilon}_{j}\right) \cdot r=\left(\varepsilon_{j+1}-\varepsilon_{j}\right) \cdot p=\beta_{j} \cdot r^{a} \cdot p, \quad j=1, \ldots, \mu .
$$

3.4.5. The weight on $\mathscr{L}_{\mathbb{S}}^{1}$. By (6) and our assumptions,

$$
\begin{aligned}
\mu_{\mathscr{L}_{\mathbb{S}}^{1}}(\lambda, S) & =-\sum_{i=1}^{l+1} \widetilde{\gamma}_{i} \cdot\left(h^{0}\left(X, E_{i}(m+n)\right)-h^{0}\left(X, E_{i-1}(m+n)\right)\right) \\
& =-\sum_{i=1}^{l+1} \widetilde{\gamma}_{i} \cdot h^{0}\left(X, E_{i}(m+n)\right)+\sum_{i=1}^{l+1} \widetilde{\gamma}_{i} \cdot h^{0}\left(X, E_{i-1}(m+n)\right) \\
& \stackrel{E_{0}=\{0\}}{=}-\sum_{i=1}^{l+1} \widetilde{\gamma}_{i} \cdot h^{0}\left(X, E_{i}(m+n)\right)+\sum_{i=1}^{l} \widetilde{\gamma}_{i+1} \cdot h^{0}\left(X, E_{i}(m+n)\right) \\
\stackrel{E_{l+1}=E}{=} & \sum_{i=1}^{l} \alpha_{i} \cdot p \cdot h^{0}\left(X, E_{i}(m+n)\right)-\widetilde{\gamma}_{l+1} \cdot p_{m} .
\end{aligned}
$$

Now,

$$
\tilde{\gamma}_{l+1}=\sum_{i=1}^{l} \alpha_{i} \cdot h^{0}\left(X, E_{i}(n)\right)
$$


Altogether

$$
\mu_{\mathscr{L}_{\overparen{S}}^{1}}(\lambda, S)=\sum_{i=1}^{l} \alpha_{i} \cdot\left(p \cdot h^{0}\left(X, E_{i}(m+n)\right)-p_{m} \cdot h^{0}\left(X, E_{i}(n)\right)\right) .
$$

Since $p_{m}=r \cdot m+p$ and, by our assumptions, $h^{0}\left(X, E_{i}(m+n)\right)=\operatorname{rk}\left(E_{i}\right) \cdot m+h^{0}\left(X, E_{i}(n)\right)$, we find

$$
\begin{aligned}
\mu_{\mathscr{L}_{\overparen{\subseteq}}^{1}}(\lambda, S) & =m \cdot \sum_{i=1}^{l} \alpha_{i} \cdot\left(p \cdot \operatorname{rk}\left(E_{i}\right)-r \cdot h^{0}\left(X, E_{i}(n)\right)\right) \\
& =m \cdot \sum_{i=1}^{l} \alpha_{i} \cdot\left(d \cdot \operatorname{rk}\left(E_{i}\right)-\operatorname{deg}\left(E_{i}\right) \cdot r\right) \\
& =m \cdot M\left(E_{\bullet}, \alpha_{\bullet}\right) .
\end{aligned}
$$

Finally, we note

$$
\begin{aligned}
\eta \cdot \mu_{\mathscr{L}_{\Im}^{1}}(\lambda, S)= & \frac{1}{\delta} \cdot \frac{p}{r} \cdot M\left(E_{\bullet}, \alpha_{\bullet}\right)+ \\
& +\frac{p}{r} \cdot \sum_{i=1}^{l} a \cdot s \cdot \alpha_{i} \cdot \operatorname{rk}\left(E_{i}\right)-\sum_{i=1}^{l} a \cdot s \cdot \alpha_{i} \cdot h^{0}\left(X, E_{i}(n)\right) .
\end{aligned}
$$

3.4.6. The weight on $\mathscr{L}_{\mathbb{S}}^{2}$. The surjection $V^{a} \otimes \mathcal{O}_{\mathfrak{S}} \longrightarrow H_{\rho, \subseteq}$ yields a $\mathrm{GL}(V)$-equivariant morphism $\mathfrak{S} \longrightarrow \mathfrak{G r}^{p(a)}\left(V^{\otimes a}\right), \mathfrak{G r}^{p(a)}\left(V^{\otimes a}\right)$ the Graßmannian of $p(a)$-dimensional

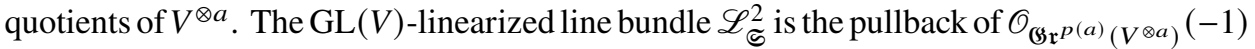
under this morphism. Therefore, Formula (6) may be used. Taking into account Remark 3.4.6, we find

$$
\mu_{\mathscr{L}_{\overleftarrow{S}}^{2}}(\lambda, S)=\sum_{j=1}^{\mu+1} \widetilde{\varepsilon}_{j} \cdot\left(h^{0}\left(X, B_{j}(a \cdot n)\right)-h^{0}\left(X, B_{j-1}(a \cdot n)\right)\right) .
$$

3.4.7. The weight on $\mathscr{L}_{\mathfrak{S}}^{3}$. Here, we can directly apply (6). Using Remark 3.4 .6 again, we see

$$
\begin{aligned}
& \mu_{\mathscr{L}_{\mathbb{E}}^{3}}(\lambda, S) \\
= & -\sum_{j=1}^{\mu+1} \widetilde{\varepsilon}_{j} \cdot\left(\operatorname{dim}_{\mathbb{C}}\left(\chi\left(H_{j}\right)\right)-\operatorname{dim}_{\mathbb{C}}\left(\chi\left(H_{j-1}\right)\right)\right) \\
= & -\sum_{j=1}^{\mu+1} \widetilde{\varepsilon}_{j} \cdot\left(\operatorname{dim}_{\mathbb{C}}\left(\widehat{\chi}\left(H^{0}\left(X, B_{j}(a n)\right)\right)\right)-\operatorname{dim}_{\mathbb{C}}\left(\widehat{\chi}\left(H^{0}\left(X, B_{j-1}(a n)\right)\right)\right)\right) \\
= & -\sum_{j=1}^{\mu+1} \widetilde{\varepsilon}_{j} \cdot\left(h^{0}\left(X, B_{j}(a \cdot n)\right)-\operatorname{dim}_{\mathbb{C}}\left(H^{0}\left(X, B_{j}(a \cdot n)\right) \cap \Gamma\right)\right. \\
& \left.-h^{0}\left(X, B_{j-1}(a \cdot n)\right)+\operatorname{dim}_{\mathbb{C}}\left(H^{0}\left(X, B_{j-1}(a \cdot n)\right) \cap \Gamma\right)\right) .
\end{aligned}
$$


3.4.8. The weight on $\mathscr{L}_{\mathbb{S}}^{2} \otimes \mathscr{L}_{\mathbb{S}}^{3}$. Combining the computations of the last two sections, we get

$$
\begin{aligned}
& \mu_{\mathscr{L}_{\mathbb{E}}^{2} \otimes \mathscr{L}_{\mathscr{E}}^{3}}(\lambda, S) \\
= & \sum_{j=1}^{\mu+1} \widetilde{\varepsilon}_{j} \cdot\left(\operatorname{dim}_{\mathbb{C}}\left(H^{0}\left(X, B_{j}(a \cdot n)\right) \cap \Gamma\right)-\operatorname{dim}_{\mathbb{C}}\left(H^{0}\left(X, B_{j-1}(a \cdot n)\right) \cap \Gamma\right)\right) .
\end{aligned}
$$

By construction of $\mathfrak{\subseteq}$, we have

$$
H^{0}\left(X, B_{j}(a \cdot n)\right) \cap \Gamma=H^{0}\left(X, B_{j}\right) \cap \Gamma, \quad j=1, \ldots, \mu+1 .
$$

We infer

$$
\begin{aligned}
& \mu_{\mathscr{L}_{\mathbb{E}}^{2} \otimes \mathscr{L}_{\mathbb{E}}^{3}}(\lambda, S) \\
= & \sum_{j=1}^{\mu+1} \widetilde{\varepsilon}_{j} \cdot \operatorname{dim}_{\mathbb{C}}\left(H^{0}\left(X, B_{j}\right) \cap \Gamma\right)-\sum_{j=1}^{\mu+1} \widetilde{\varepsilon}_{j} \cdot \operatorname{dim}_{\mathbb{C}}\left(H^{0}\left(X, B_{j-1}\right) \cap \Gamma\right) \\
\stackrel{B_{0}=\{0\}}{=} & \sum_{j=1}^{\mu+1} \widetilde{\varepsilon}_{j} \cdot \operatorname{dim}_{\mathbb{C}}\left(H^{0}\left(X, B_{j}\right) \cap \Gamma\right)-\sum_{j=1}^{\mu} \widetilde{\varepsilon}_{j+1} \cdot \operatorname{dim}_{\mathbb{C}}\left(H^{0}\left(X, B_{j}\right) \cap \Gamma\right) \\
= & \sum_{i=1}^{l} a \cdot s \cdot \alpha_{i} \cdot h^{0}\left(X, E_{i}(n)\right)-\sum_{j=1}^{\mu} \widetilde{\beta}_{j} \cdot p^{a} \cdot \operatorname{dim}_{\mathbb{C}}\left(H^{0}\left(X, B_{j}\right) \cap \Gamma\right) .
\end{aligned}
$$

For the last equality, we have used that $B_{\mu+1}=E(n)^{\otimes a}$, so that $H^{0}\left(X, B_{\mu+1}\right) \cap \Gamma=\Gamma$. Using (20), we write

$$
\mu_{\mathscr{L}_{\mathbb{S}}^{2} \otimes \mathscr{L}_{\mathbb{S}}^{3}}(\lambda, S)=\sum_{i=1}^{l} a \cdot s \cdot \alpha_{i} \cdot h^{0}\left(X, E_{i}(n)\right)-\frac{p}{r} \cdot \sum_{j=1}^{\mu} \beta_{j} \cdot r^{a} \cdot \operatorname{dim}_{\mathbb{C}}\left(H^{0}\left(X, B_{j}\right) \cap \Gamma\right) .
$$

3.4.9. The weight on $\mathcal{N}_{\eta}$. Putting (21) and (22) together, we find

$$
\begin{aligned}
\mu_{\mathcal{V}_{\eta}}(\lambda, S)= & \frac{p}{r} \cdot\left(\frac{1}{\delta} \cdot M\left(E_{\bullet}, \alpha_{\bullet}\right)+\right. \\
& \left.+\sum_{i=1}^{l} a \cdot s \cdot \alpha_{i} \cdot \operatorname{rk}\left(E_{i}\right)-\sum_{j=1}^{\mu} \beta_{j} \cdot r^{a} \cdot \operatorname{dim}_{\mathbb{C}}\left(H^{0}\left(X, B_{j}\right) \cap \Gamma\right)\right) .
\end{aligned}
$$

We multiply the right hand side by $\delta \cdot r / p$. The result is

$$
M\left(E_{\bullet}, \alpha_{\bullet}\right)+\delta \cdot\left(\sum_{i=1}^{l} a \cdot s \cdot \alpha_{i} \cdot \operatorname{rk}\left(E_{i}\right)-\sum_{j=1}^{\mu} \beta_{j} \cdot r^{a} \cdot \operatorname{dim}_{\mathbb{C}}\left(H^{0}\left(X, B_{j}\right) \cap \Gamma\right)\right) .
$$

Now, $s=\operatorname{dim}_{\mathbb{C}}(\Gamma)$ and

$$
\sum_{i=1}^{l} a \cdot \alpha_{i} \cdot \operatorname{rk}\left(E_{i}\right)=\varepsilon_{\mu+1}=\sum_{j=1}^{\mu} \beta_{j} \cdot \operatorname{rk}\left(B_{j}\right) .
$$


Abbreviating $b_{j}=\operatorname{dim}_{\mathbb{C}}\left(H^{0}\left(X, B_{j}\right) \cap \Gamma\right), j=1, \ldots, \mu+1$, as in Section 1.2, we see that

$$
\begin{aligned}
& \sum_{i=1}^{l} a \cdot s \cdot \alpha_{i} \cdot \operatorname{rk}\left(E_{i}\right)-\sum_{j=1}^{\mu} \beta_{j} \cdot r^{a} \cdot \operatorname{dim}_{\mathbb{C}}\left(H^{0}\left(X, B_{j}\right) \cap \Gamma\right) \\
= & \sum_{j=1}^{\mu} \beta_{j} \cdot\left(\operatorname{rk}\left(B_{j}\right) \cdot \operatorname{dim}_{\mathbb{C}}(\Gamma)-b_{j} \cdot r^{a}\right) \\
= & \mu_{(E, \Gamma)}\left(E_{\bullet}, \alpha_{\bullet}\right) .
\end{aligned}
$$

Altogether, we infer that $\mu_{\mathcal{N}_{\eta}}(\lambda, S)(\geq) 0$ implies

$$
M\left(E_{\bullet}, \alpha_{\bullet}\right)+\delta \cdot \mu_{(E, \Gamma)}\left(E_{\bullet}, \alpha_{\bullet}\right)(\geq) 0,
$$

as desired.

3.5. Semistability implies GIT-semistability. We now return to the more exact notation introduced at the beginning of Section 3.4. In addition, we will write $V_{n}$ for the vector space of dimension $p=d+r \cdot(n+1-g)$ used in construction of $\mathfrak{S}_{n}, n \in \mathbb{N}$. Let us fix $n$ for the moment. For a point $S=\left(\kappa: V_{n} \otimes \mathcal{O}_{X}(-n) \longrightarrow E, \Gamma\right) \in \mathbb{S}_{n}$ and a subspace $W \subset V_{n}$, we let $\mathscr{F}_{W}$ be the image of $W \otimes \mathcal{O}_{X}(-n) \longrightarrow V_{n} \otimes \mathcal{O}_{X}(-n) \stackrel{\kappa}{\longrightarrow} E$. The family of torsion free sheaves $\mathscr{F}_{W}$ obtained from a point $S=\left(\kappa: V_{n} \otimes \mathcal{O}_{X}(-n) \longrightarrow E, \Gamma\right) \in \mathfrak{S}_{n}$ and a subspace $W \subset V_{n}$ is bounded. This implies that there is a natural number $m(n)$, such that, for $m \geq m(n)$, a point $S=\left(\kappa: V_{n} \otimes \mathcal{O}_{X}(-n) \longrightarrow E, \Gamma\right) \in \mathbb{S}_{n}$, and a subspace $W \subset V_{n}$, the linear map $W \otimes H^{0}\left(X, \mathcal{O}_{X}(m)\right) \longrightarrow H^{0}\left(X, \mathscr{F}_{W}(m+n)\right)$ is surjective. Set $C_{0}:=\delta \cdot a \cdot s$. Using the notation of Section 3.4.3, we have shown in Section 3.1 that $[E] \in \mathfrak{B}_{0}\left(C_{0}\right)$. In Section 3.2, we have introduced a natural number $n_{0}$, such that $h^{1}(X, E(n))=0$, for every $\delta$-(semi)stable coherent $\varrho$-system $(E, \Gamma)$ of type $\left(\mathcal{O}_{X}, d, s\right)$ and every $n \geq n_{0}$. We have the following converse to Theorem 3.4.1.

Theorem 3.5.1. There is a natural number $n_{2} \geq n_{0}$, such that, for every $n \geq n_{2}$, every $\delta$-(semi)stable coherent $\varrho$-system $(E, \Gamma)$ of type $\left(\mathcal{O}_{X}, d, s\right)$, and every isomorphism $V_{n} \longrightarrow$ $H^{0}(X, E(n))$, the resulting point $S=\left(\kappa: V_{n} \otimes \mathcal{O}_{X}(-n) \longrightarrow E, \Gamma\right) \in \mathfrak{S}_{n}$ is (semi)stable.

Proof. Step 1. - Let $C_{4}$ be a positive real number. We look at a subspace $W \subset V_{n}$, such that $\mu\left(\mathscr{F}_{W}\right) \leq(d / r)-C_{4}$. Note that, by the Le Potier-Simpson estimate ${ }^{12}$ ([28], Lemma 7.1.2 and bottom of Page 106)

$$
\begin{aligned}
\operatorname{dim}_{\mathbb{C}}(W) & \leq h^{0}\left(X, \mathscr{F}_{W}(n)\right) \\
& \left.\leq \operatorname{rk}\left(\mathscr{F}_{W}\right)-1\right) \cdot \mu_{\max }\left(\mathscr{F}_{W}\right)+\mu\left(\mathscr{F}_{W}\right)+\operatorname{rk}\left(\mathscr{F}_{W}\right) \cdot(n+1) \\
& \leq \operatorname{rk}\left(\mathscr{F}_{W}\right) \cdot\left(\frac{d}{r}+n+1\right)+\left(\operatorname{rk}\left(\mathscr{F}_{W}\right)-1\right) \cdot C_{0}-C_{4} \\
& \leq \operatorname{rk}\left(\mathscr{F}_{W}\right) \cdot\left(\frac{d}{r}+n-C_{4}^{\prime}\right), \quad C_{4}^{\prime}:=-C_{0}-1+\frac{1}{r} \cdot C_{4} .
\end{aligned}
$$

\footnotetext{
${ }^{12}$ For this to work properly, we need that $(d / r)-C_{4}+n+1$ is non-negative. The constant $C_{4}$ that we will
} introduce below does not depend on $n$, so that $(d / r)-C_{4}+n+1 \geq 0$ will be true, for $n \gg 0$. 
We now have

$$
\begin{aligned}
& p \cdot \chi\left(X, \mathscr{F}_{W}(m+n)\right)-\operatorname{dim}_{\mathbb{C}}(W) \cdot p_{m} \\
\geq & p \cdot \chi\left(X, \mathscr{F}_{W}(m+n)\right)-\operatorname{rk}\left(\mathscr{F}_{W}\right) \cdot\left(\frac{d}{r}+n-C_{4}^{\prime}\right) \cdot p_{m} \\
= & (d+r \cdot(n+1-g)) \cdot\left(\operatorname{deg}\left(\mathscr{F}_{W}\right)+\operatorname{rk}\left(\mathscr{F}_{W}\right) \cdot(m+n+1-g)\right) \\
& -\operatorname{rk}\left(\mathscr{F}_{W}\right) \cdot\left(\frac{d}{r}+n-C_{4}^{\prime}\right) \cdot(d+r \cdot(m+n+1-g)) .
\end{aligned}
$$

Since $\mathscr{F}_{W}$ is a quotient of the semistable vector bundle $W \otimes \mathcal{O}_{X}(-n)$, we have $\mu\left(\mathscr{F}_{W}\right) \geq-n$, i.e., $\operatorname{deg}\left(\mathscr{F}_{W}\right) \geq-\operatorname{rk}\left(\mathscr{F}_{W}\right) \cdot n$. So, the above polynomial is bounded from below by

$$
\begin{aligned}
& (d+r \cdot(n+1-g)) \cdot \operatorname{rk}\left(\mathscr{F}_{W}\right) \cdot(m+1-g) \\
& -\operatorname{rk}\left(\mathscr{F}_{W}\right) \cdot\left(\frac{d}{r}+n-C_{4}^{\prime}\right) \cdot(d+r \cdot(m+n+1-g)) \\
\geq & (d+r \cdot(n+1-g)) \cdot \operatorname{rk}\left(\mathscr{F}_{W}\right) \cdot(m+1-g) \\
& -\operatorname{rk}\left(\mathscr{F}_{W}\right) \cdot\left(\frac{d}{r}+n-C_{4}^{\prime}\right) \cdot(d+r \cdot(m+1-g))-\operatorname{rk}\left(\mathscr{F}_{W}\right) \cdot\left(\frac{d}{r}-C_{4}^{\prime}\right) \cdot n-r^{2} \cdot n^{2} .
\end{aligned}
$$

Apart from the term $-r^{2} \cdot n^{2}$, this is a linear polynomial in $m$ and $n$. The coefficient of $m$ is $\operatorname{rk}\left(\mathscr{F}_{W}\right) \cdot r \cdot\left(C_{4}^{\prime}+1-g\right)$. We may assume that $C_{4}$ is so large that $C_{4}^{\prime}+1-g \geq 0$. Then, the coefficient of $m$ is bounded from below by

$$
C_{5}^{\prime}:=r \cdot\left(C_{4}^{\prime}+1-g\right) .
$$

The coefficient of $n$ is $-2 \cdot \operatorname{rk}\left(\mathscr{F}_{W}\right) \cdot d+\operatorname{rk}\left(\mathscr{F}_{W}\right) \cdot r \cdot C_{4}^{\prime} \geq-2 \cdot r \cdot \max \{0, d\}=: C_{6}^{\prime}$. Finally, the constant term is

$$
\begin{aligned}
& \operatorname{rk}\left(\mathscr{F}_{W}\right) \cdot r \cdot(1-g)^{2}-\frac{\operatorname{rk}\left(\mathscr{F}_{W}\right)}{r} \cdot d^{2}+\operatorname{rk}\left(\mathscr{F}_{W}\right) \cdot C_{4}^{\prime} \cdot(d+r \cdot(1-g)) \\
\geq & r \cdot(1-g)^{2}-d^{2}+r \cdot \min \{0, d+r \cdot(1-g)\} \cdot C_{4}^{\prime}=: C_{7}^{\prime} .
\end{aligned}
$$

Finally, we look at the expression

$$
\begin{aligned}
& (\alpha \cdot n+\beta) \cdot\left(p \cdot \chi\left(X, \mathscr{F}_{W}(m+n)\right)-\operatorname{dim}_{\mathbb{C}}(W) \cdot p_{m}\right)-m \cdot(p(a)-s) \cdot a \\
\geq & \left(\alpha \cdot C_{5}^{\prime}-r^{a} \cdot a^{2}\right) \cdot m \cdot n+\left(\beta \cdot C_{5}^{\prime}+a \cdot\left(r^{a} \cdot(1-g)+a \cdot r^{a-1} \cdot d-s\right)\right) \cdot m \\
& +\left(\alpha \cdot C_{6}^{\prime}-r^{2}\right) \cdot n^{2}+\left(\alpha \cdot C_{7}^{\prime}+\beta \cdot C_{6}^{\prime}\right) \cdot n+\beta \cdot C_{7}^{\prime} .
\end{aligned}
$$

We choose $C_{4}$, such that, say, $\alpha \cdot C_{5}^{\prime}-r^{a} \cdot a^{2}=1$. This choice depends only on $a, r, d$, and $\delta$. The remaining coefficients of the polynomial then depend only on $a, r, d, s$, and $\delta$. So, for $n \gg 0$, both

$$
\frac{n}{2}+\beta \cdot C_{5}^{\prime}+a \cdot\left(r^{a} \cdot(1-g)+a \cdot r^{a-1} \cdot d-s\right)>0
$$

and

$$
\frac{n^{3}}{2}+\left(\alpha \cdot C_{6}^{\prime}-r^{2}\right) \cdot n^{2}+\left(\alpha \cdot C_{7}^{\prime}+\beta \cdot C_{6}^{\prime}\right) \cdot n+\beta \cdot C_{7}^{\prime}>0
$$

For such an $n$ and $m \geq n^{2}$, the expression on the right hand side of Inequality (23) will be positive.

Step 2. - Let us look at a one parameter subgroup $\lambda: \mathbb{C}^{\star} \longrightarrow \operatorname{GL}\left(V_{n}\right)$. It defines a $\mathbb{Q}_{>0}$-weighted flag $\left(W_{\bullet}, \vartheta_{\bullet}\right)$ with $W_{\bullet}:\{0\} \subsetneq W_{1} \subsetneq \cdots \subsetneq W_{l} \subsetneq V_{n}$ and $\vartheta_{\bullet}=\left(\vartheta_{1}, \ldots, \vartheta_{l}\right)$. 
Let us write $\{1, \ldots, l\}=I^{\prime} \sqcup I^{\prime \prime}$ with $I^{\prime}=\left\{i_{1}, \ldots, i_{l^{\prime}}\right\}$ and $I^{\prime \prime}=\left\{j_{1}, \ldots, j_{l^{\prime \prime}}\right\}$. We now introduce the weighted filtration $\left(W_{\bullet}^{\prime}, \vartheta_{\bullet}^{\prime}\right)$ with

$$
W_{\bullet}^{\prime}: \quad\{0\} \subsetneq W_{i_{1}} \subsetneq \cdots \subsetneq W_{i_{l^{\prime}}} \subsetneq V_{n}, \quad \vartheta_{\bullet}^{\prime}=\left(\vartheta_{i_{1}}^{\prime}, \ldots, \vartheta_{i_{l^{\prime}}}^{\prime}\right)
$$

and the weighted filtration $\left(W_{\bullet}^{\prime \prime}, \vartheta_{\bullet}^{\prime \prime}\right)$ with

$$
W_{\bullet}^{\prime \prime}: \quad\{0\} \subsetneq W_{j_{1}} \subsetneq \cdots \subsetneq W_{j_{l^{\prime \prime}}} \subsetneq V_{n}, \quad \vartheta_{\bullet}^{\prime \prime}=\left(\vartheta_{j_{1}}^{\prime \prime}, \ldots, \vartheta_{j_{l^{\prime \prime}}}^{\prime \prime}\right)
$$

We choose an appropriate basis $\left(v_{1}, \ldots, v_{p}\right)$ for $V_{n}$, such that $\lambda$ is defined with respect to that basis by the weight vector $\sum_{i=1}^{l} \vartheta_{i} \cdot g_{p}^{\left(\operatorname{dim}_{\mathbb{C}}\left(W_{i}\right)\right)}$. Let $\lambda^{\prime}$ and $\lambda^{\prime \prime}$ be the one parameter subgroups that are defined with respect to the basis $\left(v_{1}, \ldots, v_{p}\right)$ by the weight vectors $\sum_{h=1}^{l^{\prime}} \vartheta_{i_{h}} \cdot g_{p}^{\left(\operatorname{dim}_{\mathbb{C}}\left(W_{i_{h}}\right)\right)}$ and $\sum_{k=1}^{l^{\prime \prime}} \vartheta_{j_{k}} \cdot g_{p}^{\left(\operatorname{dim}_{\mathbb{C}}\left(W_{j_{k}}\right)\right)}$, respectively. Then, $\lambda=\lambda^{\prime}+\lambda^{\prime \prime}$, and the $\mathbb{Q}_{>0}$-weighted flag of $\lambda^{\prime}$ and $\lambda^{\prime \prime}$ is $\left(W_{\bullet}^{\prime}, \vartheta_{\bullet}^{\prime}\right)$ and $\left(W_{\bullet}^{\prime \prime}, \vartheta_{\bullet}^{\prime \prime}\right)$, respectively. Using the notation from the proof of Proposition 3.4.2, we have, for $m \geq m(n)$,

$$
\begin{aligned}
& \mu_{\left(\mathscr{L}_{\mathbb{E}_{n}}^{1}\right)^{\otimes \eta(m)} \otimes \mathscr{L}_{\mathbb{E}_{n}}^{2}}(\lambda, S) \\
= & \frac{p}{m} \cdot(\alpha \cdot n+\beta) \cdot \mu_{\mathscr{L}_{\mathbb{S}_{n}}^{1}(m)}(\lambda, S) \\
= & \frac{p}{m} \cdot(\alpha \cdot n+\beta) \cdot \sum_{i=1}^{l} \vartheta_{i} \cdot\left(p \cdot \chi\left(X, \mathscr{F}_{W_{i}}(m+n)\right)-\operatorname{dim}_{\mathbb{C}}\left(W_{i}\right) \cdot p_{m}\right) \\
= & \frac{p}{m} \cdot(\alpha \cdot n+\beta) \cdot \sum_{h=1}^{l^{\prime}} \vartheta_{i_{h}} \cdot\left(p \cdot \chi\left(X, \mathscr{F}_{W_{i_{h}}}(m+n)\right)-\operatorname{dim}_{\mathbb{C}}\left(W_{i_{h}}\right) \cdot p_{m}\right) \\
& +\frac{p}{m} \cdot(\alpha \cdot n+\beta) \cdot \sum_{k=1}^{l^{\prime \prime}} \vartheta_{j_{k}} \cdot\left(p \cdot \chi\left(X, \mathscr{F}_{W_{j_{k}}}(m+n)\right)-\operatorname{dim}_{\mathbb{C}}\left(W_{j_{k}}\right) \cdot p_{m}\right) \\
= & \mu_{\left(\mathscr{L}_{\mathbb{S}_{n}}^{1}\right)^{\otimes \eta(m)} \otimes \mathscr{L}_{\mathbb{E}_{n}}^{2}}\left(\lambda^{\prime}, S\right) \\
& +\frac{p}{m} \cdot(\alpha \cdot n+\beta) \cdot \sum_{k=1}^{l^{\prime \prime}} \vartheta_{j_{k}} \cdot\left(p \cdot \chi\left(X, \mathscr{F}_{W_{j_{k}}}(m+n)\right)-\operatorname{dim}_{\mathbb{C}}\left(W_{j_{k}}\right) \cdot p_{m}\right) .
\end{aligned}
$$

By means of the Plücker embedding, we have

$$
\Gamma: \bigwedge_{n}^{p(a)-s} V_{n}^{\otimes a} \longrightarrow \mathbb{C}
$$

Since the exterior power is a direct summand of the tensor power, we get

$$
\widetilde{\Gamma}: V_{n}^{\otimes(a \cdot(p(a)-s))} \longrightarrow \mathbb{C} .
$$

We use [35], Lemma 1.5.1.41, to infer

$$
\mu_{\mathscr{L}_{\widetilde{\Phi}_{n}^{3}}^{3}}(\lambda, S) \geq \mu_{\mathscr{L}_{\widetilde{\Phi}_{n}^{3}}^{3}}\left(\lambda^{\prime}, S\right)-\left(\sum_{k=1}^{l^{\prime \prime}} \vartheta_{j_{k}}\right) \cdot(p(a)-s) \cdot a \cdot p
$$


Altogether, we see that

$$
\begin{aligned}
& \mu_{\mathcal{N}_{\eta(m)}}(\lambda, S) \\
\geq & \mu_{\mathcal{N}_{\eta(m)}}\left(\lambda^{\prime}, S\right) \\
& +\frac{p}{m} \cdot\left(\sum_{k=1}^{l^{\prime \prime}} \vartheta_{j_{k}} \cdot\left(p \cdot \chi\left(X, \mathscr{F}_{W_{j_{k}}}(m+n)\right)-\operatorname{dim}_{\mathbb{C}}\left(W_{j_{k}}\right) \cdot p_{m}-m \cdot(p(a)-s) \cdot a\right)\right) .
\end{aligned}
$$

Now, we choose $C_{4}$ as in Step 1 and assume that $n$ is large enough and that $m \geq$ $\max \left\{n^{2}, m(n)\right\}$. Then, we define

$$
I^{\prime}:=\left\{v \in\{1, \ldots, l\} \mid \mu\left(\mathscr{F}_{W_{v}}\right) \leq \frac{d}{r}-C_{4}\right\} \quad \text { and } \quad I^{\prime \prime}:=\{1, \ldots, l\} \backslash I^{\prime} .
$$

If $I^{\prime \prime} \neq \varnothing$, Step 1 shows

$$
\sum_{k=1}^{l^{\prime \prime}} \vartheta_{j_{k}} \cdot\left(p \cdot \chi\left(X, \mathscr{F}_{W_{j_{k}}}(m+n)\right)-\operatorname{dim}_{\mathbb{C}}\left(W_{j_{k}}\right) \cdot p_{m}-m \cdot(p(a)-s) \cdot a\right)>0,
$$

so that

$$
\mu_{\mathcal{N}_{\eta(m)}}(\lambda, S)>\mu_{\mathcal{N}_{\eta(m)}}\left(\lambda^{\prime}, S\right)
$$

Step 3. - If $(E, \Gamma)$ is a $\delta$-semistable coherent system of type $\left(\mathcal{O}_{X}, d, s\right)$, then, as recalled at the beginning of this section, $[E]$ belongs to the bounded family $\mathfrak{B}_{0}\left(C_{0}\right)$. We choose $C_{4}$ as in Step 1. We will assume that $\mathfrak{B}_{1}\left(C_{0}, C_{4}\right) \subset \mathfrak{B}$ and that $n$ is so large that the conditions presented at the beginning of Section 3.2 are satisfied.

Remark 3.5.2. Let $E$ be a vector bundle of rank $r$ and degree $d$ with $\mu_{\max }(E) \leq d / r+C_{0}$, $\{0\} \subsetneq \mathscr{F} \subset E$ a subsheaf with $\mu(\mathscr{F}) \geq d / r-C_{4}$. Then, there are only finitely many possibilities for $\operatorname{deg}(\mathscr{F})$. Moreover, $\mu_{\max }(\mathscr{F}) \leq d / r+C_{0}$. Therefore, the family of all locally free $\mathcal{O}_{X}$-modules $\mathscr{F}$, such that $\mu(\mathscr{F}) \geq d / r-C_{4}$ and $\mathscr{F}$ is isomorphic to a subsheaf of a vector bundle $E$ with $[E] \in \mathfrak{B}_{0}\left(C_{0}\right)$ is bounded, too. We may assume that this family forms part of $\mathfrak{B}$. In particular, we may assume that $H^{1}(X, \mathscr{F}(n))=0$ for all these sheaves and $n \gg 0$.

Let us look at a one parameter subgroup $\lambda: \mathbb{C}^{\star} \longrightarrow \mathrm{GL}\left(V_{n}\right)$ and its $\mathbb{Q}_{>0}$-weighted flag $\left(W_{\bullet}, \vartheta_{\bullet}\right)$ with $W_{\bullet}:\{0\} \subsetneq W_{1} \subsetneq \cdots \subsetneq W_{l} \subsetneq V_{n}$ and $\vartheta_{\bullet}=\left(\vartheta_{1}, \ldots, \vartheta_{l}\right)$. By Step 2, we may assume that $\mu\left(\mathscr{F}_{W_{i}}\right) \geq(d / r)-C_{4}, i=1, \ldots, l$. Let $F_{W_{i}} \subset E$ be the subbundle generated by $\mathscr{F}_{W_{i}}, i=1, \ldots, l$. We get the filtration

$$
\{0\} \subseteq F_{W_{1}} \subseteq F_{W_{2}} \subseteq \cdots \subseteq F_{W_{l}} \subseteq E .
$$

We remove the improper inclusions and arrive at the filtration

$$
E_{\bullet}: \quad\{0\} \subsetneq E_{1} \subsetneq \cdots \subsetneq E_{\bar{l}} \subsetneq E .
$$

Let

$$
\tau:\{1, \ldots, l+1\} \longrightarrow\{1, \ldots, \bar{l}+1\}
$$

be the map defined by the rule

$$
\forall i \in\{1, \ldots, l+1\} \forall j \in\{1, \ldots, \bar{l}+1\}: \quad \tau(i)=j \quad \Longleftrightarrow \quad F_{W_{i}}=E_{j},
$$

using $W_{l+1}:=V_{n}$ and $E_{\bar{l}+1}:=E$. Also define

$$
T(j):=\{i \in\{1, \ldots, l\} \mid \tau(i)=j\}, \quad j=1, \ldots, \bar{l}+1 .
$$


As before, we let $\left(v_{1}, \ldots, v_{p}\right)$ be a basis of $V_{n}$, such that the image of $\left\langle v_{1}, \ldots, v_{\chi}\left(X, E_{j}(n)\right)\right\rangle$ under the isomorphism $H^{0}(X, \kappa(n))$ is $H^{0}\left(X, E_{j}(n)\right), j=1, \ldots, \bar{l}^{13}$ Next, for $j=1, \ldots, \bar{l}$, we set

$$
\alpha_{j}:=\sum_{i \in T(j)} \vartheta_{i}
$$

Now, let $\bar{\lambda}$ be the one parameter subgroup that is defined with respect to the basis $\left(v_{1}, \ldots, v_{p}\right)$ by the weight vector $\sum_{j=1}^{\bar{l}} \alpha_{j} \cdot g_{p}^{\left(\chi\left(X, E_{j}(n)\right)\right)}$. The $\mathbb{Q}_{>0}$-weighted flag of $\bar{\lambda}$ features

$\bar{W}_{\bullet}:\{0\} \subsetneq H^{0}(X, \kappa(n))^{-1}\left(H^{0}\left(X, E_{1}(n)\right)\right) \subsetneq \cdots \subsetneq H^{0}(X, \kappa(n))^{-1}\left(H^{0}\left(X, E_{\bar{l}}(n)\right)\right) \subsetneq V_{n}$ and $\alpha_{\bullet}=\left(\alpha_{1}, \ldots, \alpha_{\bar{l}}\right)$.

Claim. $\mu_{\mathscr{N}_{\eta(m)}}(\lambda, S) \geq \mu_{\mathcal{N}_{\eta(m)}}(\bar{\lambda}, S)$.

With this claim, we will be done, because, for $\mu_{\mathcal{N}_{\eta(m)}}(\bar{\lambda}, S)$, the computation in Section 3.4.4 can be just done backwards to deduce $\mu_{\mathcal{N}_{\eta(m)}}(\bar{\lambda}, S)(\geq) 0$ from $M\left(E_{\bullet}, \alpha \bullet\right)+\delta$. $\mu_{(E, \Gamma)}\left(E_{\bullet}, \alpha_{\bullet}\right)(\geq) 0$.

First, we note that

$$
\mu_{\mathscr{L}_{\mathscr{S}}^{1}}(\lambda, S)=\sum_{i=1}^{l} \vartheta_{i} \cdot\left(p \cdot \chi\left(X, \mathscr{F}_{W_{i}}(m+n)\right)-\operatorname{dim}_{\mathbb{C}}\left(W_{i}\right) \cdot p_{m}\right) .
$$

We have $\chi\left(X, \mathscr{F}_{W_{i}}(m+n)\right)=\operatorname{rk}\left(\mathscr{F}_{W_{i}}\right) \cdot m+\chi\left(X, \mathscr{F}_{W_{i}}(n)\right), i=1, \ldots, l$, so that we infer

$$
\begin{aligned}
\mu_{\mathscr{L}_{\mathscr{S}}^{1}}(\lambda, S)= & m \cdot \sum_{i=1}^{l} \vartheta_{i} \cdot\left(p \cdot \operatorname{rk}\left(\mathscr{F}_{W_{i}}\right)-\operatorname{dim}_{\mathbb{C}}\left(W_{i}\right) \cdot r\right) \\
& +\sum_{i=1}^{l} \vartheta_{i} \cdot\left(p \cdot \chi\left(X, \mathscr{F}_{W_{i}}(n)\right)-\operatorname{dim}_{\mathbb{C}}\left(W_{i}\right) \cdot p\right) .
\end{aligned}
$$

According to Remark 3.5.2, $\chi\left(X, \mathscr{F}_{W_{i}}(n)\right)=h^{0}\left(X, \mathscr{F}_{W_{i}}(n)\right) \geq \operatorname{dim}_{\mathbb{C}}\left(W_{i}\right), i=1, \ldots, l$. It follows that

$$
\mu_{\mathscr{L}_{\mathscr{E}}^{1}}(\lambda, S) \geq m \cdot \sum_{i=1}^{l} \vartheta_{i} \cdot\left(p \cdot \operatorname{rk}\left(\mathscr{F}_{W_{i}}\right)-\operatorname{dim}_{\mathbb{C}}\left(W_{i}\right) \cdot r\right) .
$$

This yields

$$
\begin{aligned}
\eta(m) \cdot \mu_{\mathscr{L}_{\mathscr{S}}^{1}}(\lambda, S) \geq & \frac{1}{\delta} \cdot \frac{p}{r} \cdot \sum_{i=1}^{l} \vartheta_{i} \cdot\left(p \cdot \operatorname{rk}\left(\mathscr{F}_{W_{i}}\right)-\operatorname{dim}_{\mathbb{C}}\left(W_{i}\right) \cdot r\right) \\
& +\frac{p}{r} \cdot \sum_{i=1}^{l} a \cdot s \cdot \vartheta_{i} \cdot \operatorname{rk}\left(\mathscr{F}_{W_{i}}\right)-\sum_{i=1}^{l} a \cdot s \cdot \vartheta_{i} \cdot \operatorname{dim}_{\mathbb{C}}\left(W_{i}\right)
\end{aligned}
$$

For $i=1, \ldots, l, \operatorname{rk}\left(\mathscr{F}_{W_{i}}\right)=\operatorname{rk}\left(E_{\tau(i)}\right)$ and $\operatorname{dim}_{\mathbb{C}}\left(W_{i}\right) \leq h^{0}\left(X, \mathscr{F}_{W_{i}}(n)\right) \leq h^{0}\left(X, F_{W_{i}}(n)\right)=$ $h^{0}\left(X, E_{\tau(i)}(n)\right)=\chi\left(X, E_{\tau(i)}(n)\right)$, so that

$$
p \cdot \operatorname{rk}\left(\mathscr{F}_{W_{i}}\right)-\operatorname{dim}_{\mathbb{C}}\left(W_{i}\right) \cdot r \geq d \cdot \operatorname{rk}\left(E_{\tau(i)}\right)-\operatorname{deg}\left(E_{\tau(i)}\right) \cdot r .
$$

${ }^{13}$ Recall that we assume that $\mathfrak{B}_{1}\left(C_{0}, C_{4}\right) \subset \mathfrak{B}$. This implies $H^{1}(X, F(n))=0$, for a vector bundle $F$ with $[F] \in \mathfrak{B}$. In particular, we have $h^{0}\left(X, E_{j}(n)\right)=\chi\left(X, E_{j}(n)\right), j=1, \ldots, \bar{l}$. 
So, we arrive at the estimate

$$
\begin{aligned}
\eta(m) \cdot \mu_{\mathscr{L}_{\overparen{\Xi}}}(\lambda, S) \geq & \frac{1}{\delta} \cdot \frac{p}{r} \cdot \sum_{i=1}^{l} \vartheta_{i} \cdot\left(d \cdot \operatorname{rk}\left(E_{\tau(i)}\right)-\operatorname{deg}\left(E_{\tau(i)}\right) \cdot r\right) \\
& +\frac{p}{r} \cdot \sum_{i=1}^{l} a \cdot s \cdot \vartheta_{i} \cdot \operatorname{rk}\left(E_{\tau(i)}\right)-\sum_{i=1}^{l} a \cdot s \cdot \vartheta_{i} \cdot \operatorname{dim}_{\mathbb{C}}\left(W_{i}\right) .
\end{aligned}
$$

Next, let $\gamma_{1}<\cdots<\gamma_{l+1}$ be the distinct weights forming part of $\sum_{i=1}^{l} \vartheta_{i} \cdot g_{p}^{\left(\operatorname{dim}_{\complement}\left(W_{i}\right)\right)}$. We set

$$
\widetilde{G}:=\left\{\gamma_{i_{1}}+\cdots+\gamma_{i_{a}} \mid\left(i_{1}, \ldots, i_{a}\right) \in\{1, \ldots, l+1\}\right\}
$$

and let

$$
\widetilde{\varepsilon}_{1}<\cdots<\widetilde{\varepsilon}_{\mu+1}
$$

be the distinct elements of $\widetilde{G}$. For $i=1, \ldots, \mu+1$, we consider

$$
U_{i}:=\sum_{\substack{\left(i_{1}, \ldots, i_{a}\right) \in\{1, \ldots, l+1\}^{\times a}: \\ \gamma_{i_{1}}+\cdots+\gamma_{i_{a}} \leq \bar{\varepsilon}_{i}}} W_{i_{1}} \otimes \cdots \otimes W_{i_{a}}
$$

and

$$
\mathscr{B}_{i}:=\sum_{\substack{\left(i_{1}, \ldots, i_{a}\right) \in\left\{1, \ldots, l+1 \\ \gamma_{i_{1}}+\cdots+\gamma_{i_{a}} \leq \widetilde{\mathcal{E}}_{i}\right.}} \mathscr{F} W_{i_{1}} \otimes \cdots \otimes \mathscr{F} W_{i_{a}} .
$$

The sum is taken within $V^{\otimes a}$ and $E^{\otimes a}$, respectively. Setting

$$
\widetilde{\beta}_{i}:=\frac{\widetilde{\varepsilon}_{i+1}-\widetilde{\varepsilon}_{i}}{p^{a}}, \quad i=1, \ldots, \mu,
$$

$\left(U_{\bullet}, \widetilde{\beta}_{\bullet}\right)$ with $U_{\bullet}:\{0\} \subsetneq U_{1} \subsetneq \cdots \subsetneq U_{\mu}$ and $\widetilde{\beta}_{\bullet}=\left(\widetilde{\beta}_{1}, \ldots, \widetilde{\beta}_{\mu}\right)$ is the $\mathbb{Q}_{>0}$-weighted flag in $V^{\otimes a}$ induced by $\left(V_{\bullet}, \vartheta_{\bullet}\right)$. In a similar fashion, we let $\zeta_{1}<\cdots<\zeta_{\bar{l}+1}$ be the disctinct entries of the weight vector $\sum_{j=1}^{\bar{l}} \alpha_{j} \cdot g_{p}^{\left(\chi\left(X, E_{j}(n)\right)\right)}$, define

$$
G:=\left\{\zeta_{j_{1}}+\cdots+\zeta_{j_{a}} \mid\left(j_{1}, \ldots, j_{a}\right) \in\{1, \ldots, \bar{l}+1\}\right\},
$$

and

$$
\bar{\varepsilon}_{1}<\cdots<\bar{\varepsilon}_{\bar{\mu}+1}
$$

as the distinct elements of $G$. For $j=1, \ldots, \bar{\mu}+1$, we declare

$$
B_{j}:=\sum_{\substack{\left(j_{1}, \ldots, j_{a}\right) \in\left\{1, \ldots, \bar{l}+1 \\ \zeta_{j_{1}}+\cdots+\zeta_{j_{a}} \leq \bar{\varepsilon}_{i}\right.}} E_{j_{1}} \otimes \cdots \otimes E_{j_{a}} .
$$

Note that the filtration

$$
B_{\bullet}: \quad\{0\} \subsetneq B_{1} \subsetneq \cdots \subsetneq B_{\bar{\mu}} \subsetneq E^{\otimes a}
$$

is the filtration of $E^{\otimes a}$ induced by the weighted filtration $\left(E_{\bullet}, \alpha_{\bullet}\right)$ of $E$.

Next, using $\mathscr{B}_{\mu+1}=E^{\otimes a}=B_{\bar{\mu}+1}$, we introduce

$$
\begin{aligned}
\tau^{\prime}:\{1, \ldots, \mu+1\} & \longrightarrow\{1, \ldots, \bar{\mu}+1\} \\
i & \longmapsto \min \left\{j=1, \ldots, \bar{\mu}+1 \mid \mathscr{B}_{i} \subset B_{j}\right\}
\end{aligned}
$$

and

$$
T^{\prime}(j):=\left\{i \in\{1, \ldots, l\} \mid \tau^{\prime}(i)=j\right\}, \quad j=1, \ldots, \bar{\mu}+1
$$


We point out that

$$
\bar{\beta}_{j}:=\frac{\bar{\varepsilon}_{j+1}-\bar{\varepsilon}_{j}}{p^{a}}=\sum_{i \in T^{\prime}(j)} \widetilde{\beta}_{i}, \quad j=1, \ldots, \bar{\mu} .
$$

For $i \in\{1, \ldots, \mu+1\}$, we let $H_{i}$ be the image of $U_{i}$ in $H^{0}\left(X, \mathscr{B}_{i}(a \cdot n)\right)$. As in Sections 3.4.6 to 3.4.8, we compute

$$
\mu_{\mathscr{L}_{\widetilde{E}_{n}}^{2} \otimes \mathscr{L}_{\widetilde{\Phi}_{n}}^{3}}(\lambda, S)=\sum_{i=1}^{l} a \cdot s \cdot \vartheta_{i} \cdot \operatorname{dim}_{\mathbb{C}}\left(W_{i}\right)-\sum_{i=1}^{\mu} \widetilde{\beta}_{j} \cdot p^{a} \cdot \operatorname{dim}_{\mathbb{C}}\left(H_{i} \cap \Gamma\right) .
$$

The last formula and (24) show that

$$
\begin{aligned}
& \mu_{\mathcal{N}_{\eta(m)}}(\lambda, S) \\
\geq & \frac{1}{\delta} \cdot \frac{p}{r} \cdot \sum_{i=1}^{l} \vartheta_{i} \cdot\left(d \cdot \operatorname{rk}\left(E_{\tau(i)}\right)-\operatorname{deg}\left(E_{\tau(i)}\right) \cdot r\right) \\
& +\frac{p}{r} \cdot \sum_{i=1}^{l} a \cdot s \cdot \vartheta_{i} \cdot \operatorname{rk}\left(E_{\tau(i)}\right)-\sum_{i=1}^{\mu} \widetilde{\beta}_{j} \cdot p^{a} \cdot \operatorname{dim}_{\mathbb{C}}\left(H_{i} \cap \Gamma\right) .
\end{aligned}
$$

Now,

$$
\begin{aligned}
\sum_{i=1}^{l} \vartheta_{i} \cdot\left(d \cdot \operatorname{rk}\left(E_{\tau(i)}\right)-\operatorname{deg}\left(E_{\tau(i)}\right) \cdot r\right) & =\sum_{j=1}^{\bar{l}} \alpha_{j} \cdot\left(d \cdot \operatorname{rk}\left(E_{j}\right)-\operatorname{deg}\left(E_{j}\right) \cdot r\right) \\
\sum_{i=1}^{l} a \cdot s \cdot \vartheta_{i} \cdot \operatorname{rk}\left(E_{\tau(i)}\right) & =\sum_{j=1}^{\bar{l}} a \cdot s \cdot \alpha_{j} \cdot \operatorname{rk}\left(E_{j}\right)+a \cdot s \cdot \sum_{i \in T(\bar{l}+1)} \vartheta_{i} \cdot r \\
\sum_{i=1}^{\mu} \widetilde{\beta}_{j} \cdot p^{a} \cdot \operatorname{dim}_{\mathbb{C}}\left(H_{i} \cap \Gamma\right) \leq & \sum_{i=1}^{\mu} \widetilde{\beta}_{j} \cdot p^{a} \cdot \operatorname{dim}_{\mathbb{C}}\left(H^{0}\left(X, \mathscr{B}_{\tau^{\prime}(i)}(a \cdot n)\right) \cap \Gamma\right) \\
\leq & \sum_{j=1}^{\bar{\mu}} \bar{\beta}_{j} \cdot p^{a} \cdot \operatorname{dim}_{\mathbb{C}}\left(H^{0}\left(X, B_{j}(a \cdot n)\right) \cap \Gamma\right) \\
& +s \cdot \sum_{i \in T(\bar{\mu}+1)} p^{a} \cdot \widetilde{\beta}_{i} \\
= & \sum_{j=1}^{\bar{\mu}} \bar{\beta}_{j} \cdot p^{a} \cdot \operatorname{dim}_{\mathbb{C}}\left(H^{0}\left(X, B_{j}\right) \cap \Gamma\right) \\
& +s \cdot \sum_{i \in T(\bar{\mu}+1)} p^{a} \cdot \widetilde{\beta}_{i} .
\end{aligned}
$$

Finally, we verify that

$$
a \cdot \sum_{i \in T(\bar{l}+1)} p \cdot \vartheta_{i}=\sum_{i \in T(\bar{\mu}+1)} p^{a} \cdot \widetilde{\beta}_{i}
$$

For this, observe that, for $l_{0}:=\min (T(\bar{l}+1))$, there is the identity

$$
\sum_{i \in T(\bar{l}+1)} p \cdot \vartheta_{i}=\gamma_{l+1}-\gamma_{l_{0}}
$$


Since $a \cdot \gamma_{l_{0}}$ is the weight of $\mathscr{F}_{W_{l_{0}}}^{\otimes a}$ and this subsheaf generates $E^{\otimes a}$, this weight belongs to $T^{\prime}(\bar{\mu}+1)$. On the other hand,

$$
B_{\bar{\mu}}=\sum_{k=1}^{a} E^{\otimes(k-1)} \otimes E_{\bar{l}} \otimes E^{\otimes(a-k)} .
$$

It is now straightforward to check that, for an index $i \in\{1, \ldots, \mu\}$ with $\widetilde{\varepsilon}_{i}<a \cdot \gamma_{l_{0}}$, we have $\mathscr{B}_{i} \subset B_{\bar{\mu}}$, i.e., $\tau(i) \leq \bar{\mu}$. As before, we have

$$
\sum_{i \in T(\bar{\mu}+1)} p^{a} \cdot \widetilde{\beta}_{i}=a \cdot \gamma_{l+1}-a \cdot \gamma_{l_{0}} .
$$

Our arguments prove that

$$
\begin{aligned}
\mu_{\mathcal{N}_{\eta(m)}}(\lambda, S) \geq & \frac{1}{\delta} \cdot \frac{p}{r} \cdot \sum_{j=1}^{\bar{l}} \alpha_{i} \cdot\left(d \cdot \operatorname{rk}\left(E_{j}\right)-\operatorname{deg}\left(E_{j}\right) \cdot r\right) \\
& +\frac{p}{r} \cdot \sum_{j=1}^{\bar{l}} a \cdot s \cdot \alpha_{j} \cdot \operatorname{rk}\left(E_{j}\right)-\sum_{j=1}^{\bar{\mu}} \bar{\beta}_{j} \cdot p^{a} \cdot \operatorname{dim}_{\mathbb{C}}\left(H^{0}\left(X, B_{j}\right) \cap \Gamma\right) \\
= & \mu_{\mathcal{N}_{\eta(m)}}(\bar{\lambda}, S) .
\end{aligned}
$$

This finishes the proof of the claim.

3.6. The quotient and conclusion of the proof. For $n \in \mathbb{N}$, let $\mathfrak{Q}_{n}$ be the quasi-projective quot scheme used in the construction of $\mathfrak{S}_{n}$. It is an open subscheme of a projective quot scheme $\overline{\mathfrak{Q}}_{n}$. We have constructed $\mathfrak{S}_{n}$ as a closed subscheme of $\mathfrak{I}_{n}=\mathfrak{Q}_{n} \times \mathfrak{G}_{n}$. Let $\overline{\mathfrak{S}}_{n}$ be the closure of $\mathfrak{S}_{n}$ inside the projective scheme $\overline{\mathfrak{I}}_{n}=\overline{\mathfrak{Q}}_{n} \times \mathfrak{G}_{n}$ (compare Section 3.3.1). The action of $\operatorname{GL}\left(V_{n}\right)$ on $\mathfrak{S}_{n}$ is induced by an action of $\operatorname{GL}\left(V_{n}\right)$ on $\overline{\mathfrak{I}}_{n}$, and the $\operatorname{GL}\left(V_{n}\right)$-linearized line bundle $\mathcal{N}_{\eta(m)}$ is the restriction of an ample $\mathrm{GL}\left(V_{n}\right)$-linearized line bundle $\overline{\mathcal{N}}_{\eta(m)}$ on $\overline{\mathfrak{T}}_{n}$. Let $\overline{\mathfrak{T}}_{n}^{\mathrm{ss}}$ be the set of points that are semistable with respect to the linearization in $\overline{\mathcal{N}}_{\eta(m)}$. In Theorem 3.4.1 and 3.5.1, we have shown that, for $n \gg 0$, $\mathfrak{S}_{n}^{\text {ss }}:=\mathfrak{S}_{n} \cap \overline{\mathfrak{I}}_{n}^{\text {ss }}$ consists of those points $\left(\kappa: V_{n} \otimes \mathcal{O}_{X}(-n) \longrightarrow E, \Gamma\right)$ for which $(E, \Gamma)$ is a $\delta$-semistable coherent $\varrho$-system of type $\left(\mathcal{O}_{X}, d, s\right)$.

Proposition 3.6.1. For $n \gg 0$,

$$
\mathfrak{S}_{n}^{\mathrm{ss}}=\overline{\mathfrak{S}}_{n} \cap \overline{\mathfrak{T}}_{n}^{\mathrm{ss}}
$$

Proof. In view of the arguments in the proof of Proposition 3.4.2, this is a straightforward adaptation of the proof of [35], Proposition 2.3.5.17.

By this proposition and Mumford's geometric invariant theory [30] (see also [31], [35], Chapter 1), the categorical quotient $\mathbb{\mathfrak { S }}^{\mathrm{ss}} / / \mathrm{GL}\left(V_{n}\right)=\overline{\mathfrak{S}}^{\mathrm{Ss}} / / \mathrm{GL}\left(V_{n}\right)$ exists as a projective scheme. The observations in Remark 3.2.2 and 3.3.1, ii), together with Theorem 3.4.1 and 3.5.1 imply that this quotient is the moduli space $\mathscr{C}_{X / \varrho / \mathscr{O}_{X} / d / s}^{\delta}$ that we have been looking for.

\section{OutLoOK}

In [36], we discussed a different approach to solving the moduli problem for general coherent systems. To this end, we constructed in [36], Section 4.2, a parameter scheme $\mathfrak{P}$ that is, in the notation of the present article, endowed with a $\left(\mathrm{GL}_{s}(\mathbb{C}) \times \mathrm{GL}(V)\right)$-action. The stack quotients $\left[\mathfrak{P} /\left(\mathrm{GL}_{s}(\mathbb{C}) \times \mathrm{GL}(V)\right)\right]$ and $[\mathfrak{\varsigma} / \mathrm{GL}(V)]$ are isomorphic as stacks. 
ALEXANDER SCHMITT

This can be used to compare the $\left(\mathrm{GL}_{s}(\mathbb{C}) \times \mathrm{GL}(V)\right)$-linearized line bundles on $\mathfrak{P}$ to the GL $(V)$-linearized line bundles on $\mathbb{S}$. We need to verify that the quotient $\mathfrak{P} / / \mathrm{GL}_{s}(\mathbb{C})$ in the sense of geometric invariant theory, which is an orbit space, coincides with the parameter scheme $\mathfrak{S}$ from this note. Then, the $\left(\mathrm{GL}_{s}(\mathbb{C}) \times \mathrm{GL}(V)\right)$-linearized line bundle $\mathscr{L}_{\mathfrak{P}}$ on $\mathfrak{P}$ that corresponds to the $\mathrm{GL}(V)$-linearized line bundle $\mathscr{L}_{\mathfrak{S}}$ on $\mathbb{S}$, in fact, descends to $\mathscr{L}_{\mathbb{S}}$ via the quotient map $\mathfrak{P} \longrightarrow \mathfrak{S}$. By [33], Section 1.3, or [35], Theorem 1.5.3.1, we know that the points in $\mathfrak{P}$ that are semistable with respect to the linearization in $\mathscr{L}_{\mathfrak{P}}$ map onto the points in $\subseteq$ that are semistable with respect to the linearization in $\mathscr{L}_{\mathfrak{S}}$ under the quotient map $\mathfrak{P} \longrightarrow \mathfrak{S}$. In [36], we also introduced a notion of semistability for general coherent systems which depends on a positive rational number $\varepsilon$. Fix $a, d$, and $s$. Based on the above observations, we expect the following picture:

- For $\varepsilon \in \mathbb{Q}_{>0}$, there is a number $\delta(\varepsilon) \in \mathbb{Q}_{>0}$, such that a coherent $\varrho$-system $(E, \Gamma)$ of type $\left(\mathcal{O}_{X}, d, s\right)$ is $\varepsilon$-semistable in the sense of [36] if and only if it is $\delta(\varepsilon)$-semistable in the sense of the present paper.

- As $\varepsilon$ ranges over $(0, \infty)$, the parameter $\delta$ ranges over $\left(0,1 /\left(a \cdot(a-1) \cdot r^{a-1}\right)\right)$. In particular, the limiting process $\varepsilon \rightarrow \infty$ corresponds to the limiting process $\delta \rightarrow 1 /\left(a \cdot(a-1) \cdot r^{a-1}\right)$.

The proof of this conjecture will involve subtle boundedness results for coherent $\varrho$-systems and will be considered in the future.

\section{REFERENCES}

[1] J. Alper, D. Halpern-Leistner, J. Heinloth, Existence of moduli spaces for algebraic stacks, arXiv:1812. $01128 \mathrm{v} 3,71 \mathrm{pp}$.

[2] E. Arbarello, M. Cornalba, Ph.A. Griffiths, J. Harris, Geometry of algebraic curves, vol. I, Grundlehren der Mathematischen Wissenschaften, vol. 267, Springer-Verlag, New York, 1985, xvi+386 pp.

[3] I. Biswas, N. Hoffmann, The line bundles on moduli stacks of principal bundles on a curve, Doc. Math. 15 (2010), 35-72

[4] I. Biswas, N. Hoffmann, Poincaré families of G-bundles on a curve, Math. Ann. 352 (2012), 133-54.

[5] M. Bolognesi, S. Brivio, Coherent systems and modular subvarieties of $\mathrm{SU}_{C}(r)$, Internat. J. Math. 23 (2012), 1250037, $23 \mathrm{pp}$.

[6] S.B. Bradlow, O. García-Prada, An application of coherent systems to a Brill-Noether problem, J. reine angew. Math. 551 (2002), 123-43.

[7] S.B. Bradlow, O. García-Prada, V. Muñoz, P.E. Newstead, Coherent systems and Brill-Noether theory, Internat. J. Math. 14 (2003), 683-733.

[8] L. Brambila-Paz, Non-emptiness of moduli spaces of coherent systems, Internat. J. Math. 19 (2008), 779-99.

[9] L. Brambila-Paz, Coherent Higgs systems, talk at Segundo Congreso Nacional de Geometría, Oaxaca 2018, Youtube, https: //www . youtube. com/watch?v=yU8t4qIpu1w, 49 min., published March 22, 2018.

[10] J.M. Drezet, M.S. Narasimhan, Groupe de Picard des variétés de modules de fibrés semi-stables sur les courbes algébriques, Invent. Math. 97 (1989), 53-94.

[11] E.B. Dynkin, Semisimple subalgebras of semisimple Lie algebras, in Five Papers on Algebra and Group Theory, American Mathematical Society Translations - Series 2, vol. 6, 1957, 111-244.

[12] E. Esteves, Separation properties of theta functions, Duke Math. J. 98 (1999), 565-93.

[13] G. Faltings, Vector bundles on curves, lectures held in Bonn 1995, notes by M. Stoll, 91 pp, http://www .mathe2 .uni-bayreuth. de/stoll/lecture-notes/vector-bundles-Faltings.pdf.

[14] G. Faltings, Stable G-bundles and projective connections, J. Algebraic Geom. 2 (1993), no. 3, 507-68.

[15] J. Fuchs, Affine Lie algebras and quantum groups. An introduction, with applications in conformal field theory, corrected reprint of the 1992 original, Cambridge Monographs on Mathematical Physics, Cambridge University Press, Cambridge, 1995, xiv+433 pp.

[16] W. Fulton, Intersection theory, second edition, Ergebnisse der Mathematik und ihrer Grenzgebiete, 3. Folge, a Series of Modern Surveys in Mathematics, vol. 2, Springer-Verlag, Berlin, 1998, xiv+470 pp.

[17] T.L. Gómez, I. Sols, Stability of conic bundles, with an appendix by I. Mundet i Riera, Internat. J. Math. 11 (2000), no. 8, 1027-55. 
[18] D. Halpern-Leistner, A. Fernandez Herrero, T. Jones, Moduli spaces of sheaves via affine Grassmannians, arXiv:2107.02172, $58 \mathrm{pp}$.

[19] R. Hartshorne, Algebraic Geometry, Graduate Texts in Mathematics, vol. 52, Springer-Verlag, New YorkHeidelberg, 1977, xvi+496 pp.

[20] G. Hein, Faltings' construction of the moduli space of vector bundles on a smooth projective curve, in Affine flag manifolds and principal bundles, 91-122, Trends Math., Birkhäuser/Springer Basel AG, Basel, 2010.

[21] J. Heinloth, Hilbert-Mumford stability on algebraic stacks and applications to $\mathscr{G}$-bundles on curves, Épijournal Géom. Algébrique 1 (2017), Art. 11, 37 pp.

[22] N. Hoffmann, The Picard group of a coarse moduli space of vector bundles in positive characteristic, Cent. Eur. J. Math. 10 (2012), 1306-13.

[23] D. Huybrechts, M. Lehn, The geometry of moduli spaces of sheaves, second edition, Cambridge Mathematical Library, Cambridge University Press, Cambridge, 2010, xviii +325 pp.

[24] A.D. King, P.E. Newstead, Moduli of Brill-Noether pairs on algebraic curves, Internat. J. Math. 6 (1995), $733-48$.

[25] F.F. Knudsen, D. Mumford, The projectivity of the moduli space of stable curves. I. Preliminaries on "det" and "Div", Math. Scand. 39 (1976), 19-55.

[26] S. Kumar, M.S. Narasimhan, A. Ramanathan, Infinite Grassmannians and moduli spaces of G-bundles, Math. Ann. 300 (1994), 41-75.

[27] J. Le Potier, Systèmes cohérents et structures de niveau, Astérisque, vol. 214, Société Mathématique de France, Paris, 1993, 143 pp.

[28] J. Le Potier, Lectures on vector bundles, translated by A. Maciocia, Cambridge Studies in Advanced Mathematics, vol. 54, Cambridge University Press, Cambridge, 1997, viii+251 pp.

[29] L. Moret-Bailly, Métriques permises, in Seminar on arithmetic bundles: the Mordell conjecture, Paris, 1983/84, Astérisque, vol. 127, Société Mathématique de France, Paris, 1985, 29-87.

[30] D. Mumford, J. Fogarty, F. Kirwan, Geometric invariant theory, third edition, Ergebnisse der Mathematik und ihrer Grenzgebiete (2), vol. 34, Springer-Verlag, Berlin, 1994, xiv+292 pp.

[31] P.E. Newstead, Introduction to moduli problems and orbit spaces, reprint of the 1978 original printed in new typeset, Tata Institute of Fundamental Research Lectures on Mathematics and Physics, vol. 51, Tata Institute of Fundamental Research, Bombay, by the Narosa Publishing House, New Delhi, 2012, x+153 pp.

[32] P.E. Newstead, Higher rank Brill-Noether theory and coherent systems: Open questions, arXiv:2108.08169, $25 \mathrm{pp}$.

[33] Ch. Okonek, A. Schmitt, A. Teleman, Master spaces for stable pairs, Topology 38 (1999), no. 1, 117-39.

[34] A. Schmitt, A universal construction for moduli spaces of decorated vector bundles over curves, Transform. Groups 9 (2004), 167-209.

[35] A. Schmitt, Geometric invariant theory and decorated principal bundles, Zurich Lectures in Advanced Mathematics, European Mathematical Society, Zürich, 2008, vii+389 pp.

[36] A. Schmitt, Notes on coherent systems, Rev. Mat. Teor. Apl. 28 (2021), no. 1, 1-38.

[37] C.S. Seshadri, Fibrés vectoriels sur les courbes algébriques, notes written by J.-M. Drezet, from a course at the École Normale Supérieure, June 1980, Astérisque, vol. 96, Société Mathématique de France, Paris, 1982, $209 \mathrm{pp}$.

[38] Ch. Sorger, Lectures on moduli of principal G-bundles over algebraic curves, in School on Algebraic Geometry (Trieste, 1999), 1-57, ICTP Lect. Notes, vol. 1, Abdus Salam Int. Cent. Theoret. Phys., Trieste, 2000 .

[39] The stacks project authors, Stacks project, https://stacks . math. columbia . edu, 2019, $6600 \mathrm{pp}$.

[40] X. Sun, Degeneration of moduli spaces and generalized theta functions, J. Algebraic Geom. 9 (2000), 459-527.

Freie Universität Berlin, Institut für Mathematik, Arnimallee 3, D-14195 Berlin, Deutschland

Email address: alexander.schmitt@fu-berlin.de 